\title{
Auction mechanisms in supply chain optimization
}

Citation for published version (APA):

Lok, R. B. (2007). Auction mechanisms in supply chain optimization. [Doctoral Thesis, Maastricht University]. Universitaire Pers Maastricht. https://doi.org/10.26481/dis.20070628rl

Document status and date:

Published: 01/01/2007

DOI:

10.26481/dis.20070628rl

Document Version:

Publisher's PDF, also known as Version of record

\section{Please check the document version of this publication:}

- A submitted manuscript is the version of the article upon submission and before peer-review. There can be important differences between the submitted version and the official published version of record.

People interested in the research are advised to contact the author for the final version of the publication, or visit the DOI to the publisher's website.

- The final author version and the galley proof are versions of the publication after peer review.

- The final published version features the final layout of the paper including the volume, issue and page numbers.

Link to publication

\footnotetext{
General rights rights.

- You may freely distribute the URL identifying the publication in the public portal. please follow below link for the End User Agreement:

www.umlib.nl/taverne-license

Take down policy

If you believe that this document breaches copyright please contact us at:

repository@maastrichtuniversity.nl

providing details and we will investigate your claim.
}

Copyright and moral rights for the publications made accessible in the public portal are retained by the authors and/or other copyright owners and it is a condition of accessing publications that users recognise and abide by the legal requirements associated with these

- Users may download and print one copy of any publication from the public portal for the purpose of private study or research.

- You may not further distribute the material or use it for any profit-making activity or commercial gain

If the publication is distributed under the terms of Article $25 \mathrm{fa}$ of the Dutch Copyright Act, indicated by the "Taverne" license above, 
Auction Mechanisms in Supply Chain Optimization

Reinder Berend Lok 
Auction Mechanisms in Supply Chain Optimization Copyright (C2007 Reinder Berend Lok

Omslagontwerp: Wouter Vuurboom

Druk: Datawyse, Maastricht

Een uitgave van Universitaire Pers Maastricht

ISBN 978-90-5278-638-4 


\title{
Auction Mechanisms in Supply Chain Optimization
}

\author{
Proefschrift
}

ter verkrijging van de graad van doctor aan de Universiteit Maastricht, op gezag van de Rector Magnificus, prof. mr. G.P.M.F. Mols, volgens het besluit van het College van Decanen, in het openbaar te verdedigen op donderdag 28 juni 2007 om 12.00 uur

door

Reinder Berend Lok 


\section{Promotor:}

Prof. dr. ir. C.P.M. van Hoesel

\section{Copromotor:}

Dr. M.D. Romero Morales (University of Oxford)

\section{Beoordelingscommissie:}

Prof. dr. R.J. Müller (voorzitter)

Dr. M. Uetz

Prof. dr. A.P.M. Wagelmans (Erasmus Universiteit Rotterdam)

Het onderzoek voor de hoofdstukken 2, 3 en 4 is financieel mede mogelijk gemaakt door de Nederlandse Organisatie voor Wetenschappelijk Onderzoek (NWO). 
Oh sometimes it's going to be lonely

Sometimes it will be sad

But I've got to keep on going

Until I hold that promised land

In the palm of my hand

Nothing ventured, nothing gained or won

Without a hard fight

We would never reach the sun

Without trying

Chris de Burgh (from Leather on my shoes, Flying Colours, 1988) 



\section{Contents}

$\begin{array}{ll}\text { Acknowledgements } & 9\end{array}$

1 Preliminaries $\quad 11$

1.1 Introduction . . . . . . . . . . . . . . . 11

1.2 Production planning in supply chains . . . . . . . . . . . . . . . 14

1.2.1 Economic ordering quantity . . . . . . . . . . . . 14

1.2.2 Economic lot-size model . . . . . . . . . . . . . . 15

1.3 Game theoretical concepts . . . . . . . . . . . . . 17

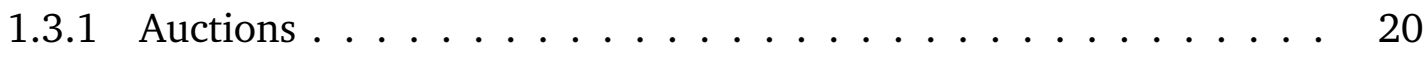

1.3.2 Mechanism design . . . . . . . . . . . . . 22

1.3.2.1 Desirable characteristics . . . . . . . . . . . 24

1.3.2.2 VCG mechanism . . . . . . . . . . . . 25

1.4 Coordination in supply chain planning: motivating examples . . . . . . 28

1.4.1 Negotiation-based planning in supply chains . . . . . . . . . . . 29

1.4.2 A bargaining model with asymmetric information . . . . . . . . 32

1.5 Outline of the thesis $\ldots \ldots \ldots \ldots \ldots$

2 Auction Procurement over Multiple Periods: Combinatorial Auction 39

2.1 Introduction . . . . . . . . . . . . . . 39

2.2 Combinatorial structure . . . . . . . . . . . . . . . . 42

2.3 Compromising setup and holding costs . . . . . . . . . . 43

2.3.1 Combinatorial VCG auction . . . . . . . . . . . . 43 
2.3.2 Stock Auction and Separate Auctions . . . . . . . . . . . . . 45

2.3.3 Comparison of the auction alternatives . . . . . . . . . . . 46

2.3.4 Combinatorial auction compromises the alternatives . . . . . 50

3 Combinatorial Auction versus Separate Auctions 53

3.1 Introduction . . . . . . . . . . . . . . . 53

3.2 Uncapacitated case . . . . . . . . . . . . . . . . . 54

3.3 Capacitated case . . . . . . . . . . . . . . 56

3.3.1 A two-period problem . . . . . . . . . . . . 57

3.3.2 Proof of nonnegative expected savings . . . . . . . . . . . 60

3.3.2.1 Setup costs ................... 60

3.3.2.2 Lower bounds . . . . . . . . . . . . . . . . 61

3.3.2.3 Density functions . . . . . . . . . . . . 65

3.3.2.4 Proof of Theorem $3.3 .3 \ldots \ldots 6$

4 Combinatorial Auction versus Stock Auction $\quad 71$

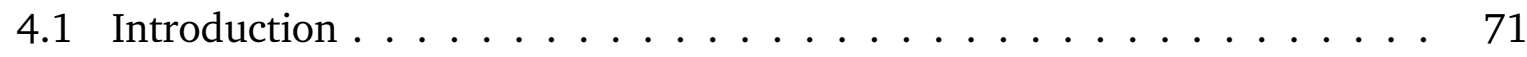

4.2 Uncapacitated case . . . . . . . . . . . . . . . . . 72

4.3 Capacitated case . . . . . . . . . . . . . . . 74

4.3.1 A two-period problem ................. 74

4.3.2 Lower bound on losses: setup costs . . . . . . . . . . . . . 78

4.3.3 Lower bound on losses: competition . . . . . . . . . . . . 80

5 Concavity and Substitutes in a Combinatorial Auction $\quad 83$

5.1 Introduction . . . . . . . . . . . . . . . 83

5.2 Cooperative games $\ldots \ldots \ldots \ldots \ldots \ldots$

5.3 The assignment problem with capacities is concave . . . . . . . . . . 86

5.4 Two applications . . . . . . . . . . . . . . . . . . 90

5.4.1 The result of Bikhchandani and Ostroy . . . . . . . . . . . 90 
5.4.2 Application to the assignment problem with capacities . . . . . . 92

5.4.3 The result of De Vries, Schummer and Vohra . . . . . . . . . . 92

5.4.4 Application to the assignment problem with capacities . . . . . . 94

5.5 Impossibilities . . . . . . . . . . . . . . . . 95

5.5.1 Generalized assignment problem . . . . . . . . . . 95

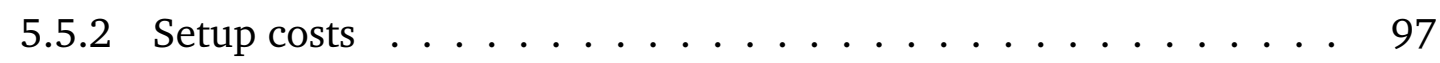

5.5.3 The VCG mechanism is not supported by anonymous prices . . . 98

6 Parametric Shortest Path Tree Problem 101

6.1 Introduction . . . . . . . . . . . . . . . . . . 101

6.2 Problem formulation . . . . . . . . . . . . . . . . 102

6.2.1 Shortest path problem .................. 102

6.2.2 Parametric shortest path tree problem . . . . . . . . . . 103

6.3 Optimality condition . . . . . . . . . . . . . . . 104

6.4 Local improvement algorithm . . . . . . . . . . . . . . . 107

6.4.1 Algorithm and example . . . . . . . . . . . . 107

6.4.2 Accelerating the algorithm . . . . . . . . . . . . 111

6.5 Alternative formulation . . . . . . . . . . . . . 113

6.5.1 Elimination of parametric variables . . . . . . . . . . . 113

6.5 .2 Open problem . . . . . . . . . . . . 117

6.6 Origin of parametric shortest path tree problems $\ldots \ldots \ldots$. . . . 117

6.6.1 Economic example: monopolist pricing . . . . . . . . . . . 117

6.6.2 Auction example: revenue maximization . . . . . . . . . . . 118

$\begin{array}{lll}7 & \text { Summary and Conclusions } & 121\end{array}$

$\begin{array}{ll}\text { Bibliography } & 125\end{array}$

$\begin{array}{ll}\text { Index } & 130\end{array}$ 


\section{Contents}

Samenvatting

135

About the author

141 


\section{Acknowledgements}

During the years of studying econometrics at the Rijksuniversiteit Groningen, it never entered my mind to start a PhD project. For sure, I very much enjoyed that time, but I also looked forward to applying the matters I learned. Only at the very end of that period some lecturers advised me to start a PhD project, but I am not the one to be driven off course that easily. However, for some reason, the idea never got out of my mind completely. After some years at Statistics Netherlands (CBS), I finally decided to look out for a PhD position, preferably in Groningen. Much to my own and others' surprise, the search leaded to Maastricht where a very interesting project was waiting.

Now, four and a half years later, the project comes to an end, and it's time to thank those who supported me during these years. First of all, I want to thank Dolores Romero Morales, my main advisor. Even after leaving Maastricht during my first year, she managed to be more involved than anybody else could be in all parts of my research. Several times I had the privilege to visit her in Oxford for a short or long stay. Those were enjoyable periods, not the least because of her and Michael's hospitality, and Dolores' art of Spanish cooking. Dolores, thanks for your inexhaustible support. Furthermore, I thank Dries Vermeulen for his help in the game theoretical aspects of my work. Especially in the first half of my research period he was of great support and we worked extensively on the research presented in chapter 5 of this thesis. I am grateful for the co-operation with Richard Steinberg. With him we worked during the last two years on the combinatorial auction as presented in the chapters 2 to 4 . Richard's knowledge on all aspects of combinatorial auctions was of great importance. With Stan van Hoesel I particularly worked on the problem presented in chapter 6 of this thesis. Stan, thanks for being my advisor and for your imperturbable happiness in all circumstances.

Besides those who were actively involved in my research, I thank Rudolf Müller for his support in mechanism design, Rakesh Vohra for providing the parametric problem, Karin van den Boorn and Haydée Hallmanns for their assistance in all but science, and finally all other colleagues and fellow $\mathrm{PhD}$ students for their pleasant company. 
Academic research consumed massive amounts of time and energy, leaving too little for the most important part of life. All the more, I am grateful that during the past few years I could be a member of the Christian congregations in Brunssum and Maastricht. It is great to share our faith and hope, and feel the love of God flowing through brothers and sisters in the Lord. Such a company makes it possible to enjoy life so far away from my family. Finally, I thank my brothers and sisters and especially my parents for their guidance in the essence of life, their confidence that I would succeed in this project, and their love and support even if I wouldn't.

Reinder Berend Lok

Maastricht, May 2007 


\title{
Chapter 1
}

\section{Preliminaries}

\author{
"But as long as you know you're nobody special, \\ you'll be a very decent sort of Horse, on the whole, \\ and taking one thing with another." \\ The Hermit in 'The Horse and His Boy' - C.S. Lewis
}

\subsection{Introduction}

This thesis focusses on incentive alignment between decision makers in supply chains. A supply chain is a sequence of actions and transactions that starts with obtaining raw materials and ends with supplying a product to consumers. This whole process can be performed within a company, but in general several companies will be involved. One company produces an intermediate product and supplies this to another company (the buyer) that uses it for further processing et cetera. For a supplier-buyer relation within such a chain the different actors have to agree on the transactions of goods between them. Our focus is on the short term operational coordination of these transactions between the links in a supply chain. There are many aspects of these transactions, but we consider only two of them, namely, production planning in relation to cost minimization and the incentives that the actors have to behave strategically. In this chapter we introduce the production problems under consideration, and the basic concepts from the field of game theory, which studies strategic interaction. Moreover, we discuss two examples where production planning and strategic behavior play a role, and which are the motivation for the rest of this thesis. But we start by motivating why it is interesting to consider the two aspects mentioned above.

Many different organizations may be involved in the supply chain for some prod- 
uct. Moreover, in a competitive environment there may be different supply chains that produce the same type of product. One of the decisive factors for the performance of a supply chain is the cooperation between the different companies involved. Furthermore, the performance of the supply chain as a whole determines the attractiveness of the produced product. However, most companies wrongly assume that behaving in their own interest is also in the interest of the supply chain:

\begin{abstract}
"Most companies don't worry about the behavior of their partners while building supply chains to deliver goods and services to consumers. (..) Every firm behaves in ways that maximize its own interests, but companies assume, wrongly, that when they do so, they also maximize the supply chain's interests. In this mistaken view, the quest for individual benefit leads to collective good, as Adam Smith argued about markets more than two centuries ago. Supply chains are expected to work efficiently without interference, as if guided by Smith's invisible hand. But our research over the last ten years shows that executives have assumed too much. We found, in more than 50 supply chains we studied, that companies often didn't act in ways that maximized the network's profits; consequently, the supply chains performed poorly." (Narayanan and Raman [34])
\end{abstract}

It is the problem of egoistical behavior of supply chain partners that we discuss in this thesis. We consider incentive schemes that reconcile individual interests and supply chain's interest. Assuming that companies are profit maximizers, companies are willing to change their behavior if it increases their individual profit. This implies that aligning all supply chain partners has some cost. Ideally, this cost is paid by the increased efficiency resulting from the alignment:

"In recent years, many companies have assumed that supply costs are more or less fixed and have fought with suppliers for a bigger share of the pie. (..) Our research, however, shows that a company can increase the size of the pie itself by aligning partners' incentives. (..) If the companies work together to efficiently deliver goods and services to consumers, they will all win. If they don't, they will all lose to another supply chain." (Narayanan and Raman [34])

Managing incentives in Supply Chain Management is on the intersection of several disciplines. Our viewpoint is from optimization problems in multi-period production planning. These are problems from the field of Operational Research. The Association 
of European Operational Research Societies (EURO) describes Operational Research as a scientific approach to the solution of problems in the management of complex systems [13]. EURO also addresses the fields of research that emerged from this approach:

"Many new analytical methods have evolved, such as: mathematical programming, simulation, game theory, queuing theory, network analysis, decision analysis, multi-criteria analysis, etc., which have powerful application to practical problems with the appropriate logical structure."

In section 1.2 we discuss two classical multi-period production planning problems. Production costs are mainly characterized by setup costs for starting a production run and costs for holding inventory. At the extremes we have either a lot of setup costs or high inventory holding costs. Costs can be minimized by finding the right balance between the different types of costs. The first classical problem is the economic ordering quantity (EOQ) model, where time runs continuously and costs and demand are constant over time. The second problem is the economic lot-size model or lot-sizing problem (LSP) where only a discrete number of periods is considered which allows for dynamic data, i.e., costs and demand that differs from period to period. Due to the dynamic data, also the other costs like unit product cost become a factor as one period may be cheaper to produce than the other.

As said before, we are interested in the transactions between a buyer and a supplier in relation to their production planning. Basically this means that we consider the question at which moments in time the supplier has to supply the products that are ordered by the buyer. Preferably, the transactions are such that total production costs in the supply chain are minimized. If there would be only one decision maker then finding the optimal transactions is just an extension of the single level problems, see for example Van Hoesel et al. [23]. However, when different decision makers are involved we get more than just an optimization problem. Then we have the problem that the goals of the decision makers might be conflicting, and not in line with a globally optimal solution. Moreover, an attempt to compromise the individual goals might be hindered by information asymmetries and the unwillingness to share information. These situations of conflict are studied in the field of game theory, of which the main concepts are discussed in section 1.3. Topics that will be treated are auctions and mechanism design.

In section 1.4 we introduce two examples where production planning and strategic behavior play a role, and which are the motivation and cohesion for the rest of this thesis. First, Dudek and Stadtler [9] consider the coordination of buyers and suppliers in the discrete dynamic setting. They propose a negotiation scheme that enables the 
supply chain partners to come close to the optimal coordinated solution. Second, we discuss the work of Sucky [49] who introduces the problem of asymmetric information in joint economic lot-sizing, i.e. the case where the EOQ problems of a buyer and a supplier are combined in one problem.

Finally, in section 1.5 an outline of the thesis is given.

\subsection{Production planning in supply chains}

In this section we present two related multi-period production planning problems. These are the basic production problems faced by individual actors in a supply chain. After discussing the related game theoretical concepts in section 1.3 we show how production planning and strategic behavior may play together in supply chains. This is done for both planning problems in section 1.4 .

\subsubsection{Economic ordering quantity}

The production problem faced by individual manufacturers can be modeled by the economic ordering quantity model (EOQ model). Consider a retailer of some divisible product. The retailer faces unit inventory holding costs $h$, and a fixed ordering cost of $f$ per order. The per unit wholesale price and the per unit selling price are constant over time. The retailer sells the product at a constant rate of $d$, i.e., in any time interval of unit length it sells $d$ units of the product. We are interested in the optimal ordering strategy for the retailer. The cost minimizing ordering quantity (that supplies all demand faced by the retailer on time) is called the economic ordering quantity (EOQ). As we assume deterministic demand, it is obvious that a new order should come in exactly when the inventory level is equal to zero. Let $Q$ be the ordering quantity, and $\tau$ the time between two orders (TBO). Then we know that $\tau=\frac{Q}{d}$, and the average inventory is $\bar{I} \equiv \frac{1}{2} Q$. So the total ordering and holding cost per unit of time equals $\left(f+\frac{1}{2} Q \tau h\right) / \tau=f d Q^{-1}+\frac{1}{2} Q h$, which is minimized if $Q=\sqrt{\frac{2 f d}{h}}$. Note that the per unit wholesale price and the per unit selling price do not directly influence the optimal value of $Q$, but there is an indirect relation as the unit inventory holding costs do depend on the value of the product. The model also has a production interpretation. In this case, we have a manufacturer facing both fixed production setup costs instead of ordering costs and unit production costs together with holding costs. In this paper we use this production interpretation. 


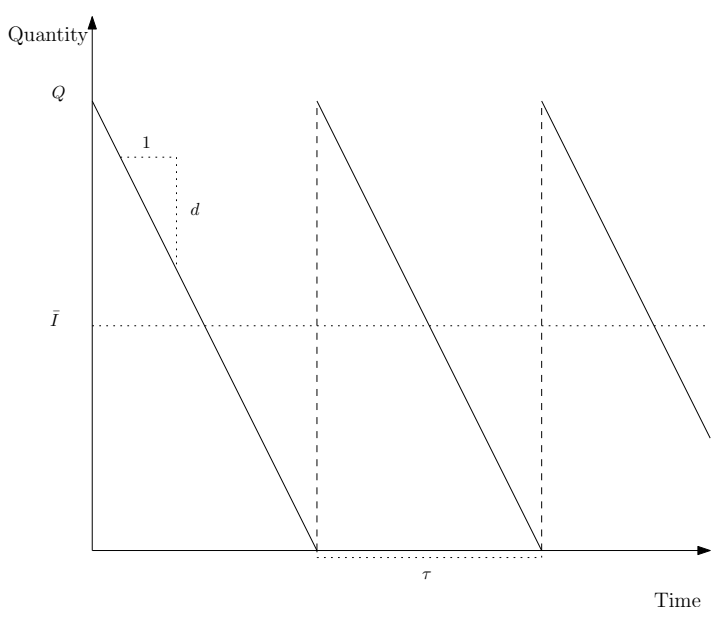

Figure 1.1: Inventory level over time (EOQ model)

The EOQ model was first presented in a paper by Harris [20]. Although published in 1913, it was apparently unnoticed for many years, as Erlenkotter [12] writes:

"Even though Harris's original paper was disseminated widely, it apparently was unnoticed for many years before its rediscovery in 1988. During this period much confusion developed over the origin of the EOQ model."

Erlenkotter [12] explores the early literature on the model and traces the evolution of the confusion.

\subsubsection{Economic lot-size model}

Wagner and Whitin [55] consider a discrete problem that is related to the EOQ model, having a finite planning horizon. This allows them to drop the assumptions of constant rate demand, constant holding costs, and constant ordering (or setup) costs, i.e. making the data dynamic. Their model is called the economic lot-size model, or single-item lot-sizing problem (LSP). Florian and Klein [14] introduced a capacitated version of this problem in which the ordering (production) quantities are limited by ordering (or production) capacity constraints. The model can be formulated as a mixed integer programming problem: Let $T$ be the planning horizon. For each period $t \in\{1, \ldots, T\}$, let $R_{t}$ be the production capacity, $p_{t}$ the unit production cost, $h_{t}$ the unit inventory holding cost, and $f_{t}$ the setup cost if production takes place. Furthermore, let $d_{t}$ be the demand for the product in period $t \in\{1, \ldots, T\}$. The lot-sizing problem for determining the 
minimum cost for delivering the demand can be formulated mathematically as follows:

$$
\min _{x, I, y} \sum_{t=1}^{T}\left(p_{t} x_{t}+h_{t} I_{t}+f_{t} y_{t}\right)
$$

subject to:

$$
\begin{aligned}
I_{t-1}+x_{t} & =d_{t}+I_{t}, \quad \forall t \in\{1, \ldots, T\}, \\
x_{t} & \leq R_{t} y_{t}, \quad \forall t \in\{1, \ldots, T\}, \\
I_{0} & =0, \\
I_{t} & \geq 0, \quad \forall t \in\{1, \ldots, T\} \\
x_{t} & \geq 0, \quad \forall t \in\{1, \ldots, T\} \\
y_{t} & \in\{0,1\}, \quad \forall t \in\{1, \ldots, T\} .
\end{aligned}
$$

Here we have for each period $t$ that $x_{t}$ is the level of production, $y_{t}$ is the setup variable, and $I_{t}$ is the level of inventory at the end of period $t$. The inventory at the beginning of period 1, i.e. $I_{0}$, is without loss of generality assumed to be zero. Constraints (1.1) are balance constraints. The inventory from the previous period plus the production in a period is either used to satisfy demand or stored for the next period. Constraints (1.2) say that production can only take place after setting up the production process. Moreover the production must not exceed the production capacity of the producer. The initial inventory equals zero by constraint (1.3). Constraints (1.4) and (1.5) say that production and inventory levels have to be non-negative numbers. The setup variables are restricted by constraints (1.6) to zero or one. Recent reviews on single-item lotsizing problems are given by Brahimi et al. [5] and Karimi et al. [27].

We are interested in the way a supplier and a buyer can coordinate their respective EOQ problems or lot-sizing problems within a supply chain. This means that we have a supplier facing a lot-sizing problem and a buyer facing a lot-sizing problem that depends on the timing of supply of the supplier. An uncoordinated solution is that first the buyer optimizes its lot-sizing problem, given the final demand; and then the buyer announces to the supplier what its input needs are to implement this optimal production plan. Then, the supplier will optimize its own lot-sizing problem, given the demand of the buyer. In a coordinated solution, the transactions between buyer and supplier are such that the total costs of both players are minimized, instead of the costs of the buyer. If the buyer and the supplier would not be part of different companies, implying that there is only one decision maker, the coordinated problem can be formulated in one two-level lot-sizing problem. In section 1.4 we discuss some approaches for finding or approaching the coordinated solution in case of different decision makers. Sucky [49] 
has an analytical approach for the EOQ model, while Dudek and Stadtler [9] have a negotiation scheme for the lot-sizing problem.

\subsection{Game theoretical concepts}

We are interested in the coordination of production planning between independent companies. As these companies may in general have conflicting interests, the coordination problem is not just an optimization problem. We have to take into account the individual and selfish behavior of the companies, and the possible strategic interaction between them. These are problems that are studied in the field of game theory, described by the Game Theory Society [16] as follows:

"Game theory studies strategic interaction in competitive and cooperative environments. Only fifty years old, it has already revolutionized economics, and is spreading rapidly to a wide variety of fields. It develops general mathematical formulas and algorithms to identify optimal strategies and to predict the outcome of interactions."

In this section we give the main notions from game theory that we use in this thesis. We start with some basic concepts. Then, in section 1.3.1 we introduce the concepts of (combinatorial) auctions. Auctions are special cases of mechanisms, i.e. games that are designed to achieve a specified outcome. In section 1.3.2 we discuss the field of mechanism design.

We start with the basic concepts from economics and game theory that we use in this thesis.

A first concept is utility. Economists usually assume that the preferences of consumers can be expressed in monetary values, i.e., consumers can express their desire for having a good in terms of an amount of money. The utility for a transaction, i.e. the reallocation of goods and money, is the net change in monetary valuation of its total possessions. For example, a person is willing to pay ten million euros for a painting of Vincent van Gogh. In an auction the person is able to buy the painting for only nine million euros. Then, the transaction of buying the painting gives a utility of one million euros. We use the term 'good' in a broad sense, i.e. including services and events. A utility function $u(\cdot)$ is a function that assigns a monetary (scalar) value to any possible transaction. Such a one-dimensional function enables us to order all possible transactions from low to high: Let $a$ and $b$ denote two different transactions, and let $a \succeq b$ 
mean that $a$ is at least as good as $b$, i.e. the utility of transaction $a$ is at least as large as the utility of transaction $b$. We say that a utility function is complete, reflexive and transitive if

- for any two transactions $a$ and $b$ : either $a \succeq b$, or $b \succeq a$, or both (complete);

- for any transaction $a$ : $a \succeq a$ (reflexive);

- for any three transactions $a, b$ and $c$ : if $a \succeq b$ and $b \succeq c$, then $a \succeq c$ (transitive).

Utility is a concept corresponding to an individual. A similar concept for a group or society of individuals is social welfare. Social welfare is naturally linked to the utilities of the individuals as it somehow compromises the utilities of all individuals into a single measure. Accordingly, a social welfare function is a function that assigns a scalar value to any vector of utilities of all individuals. However, there is not just a unique way to compromise individual utilities. For example, a socially concerned measure is the lowest individual utility, i.e. social welfare is linked to the welfare of the poorest. On the contrary, a dictator would choose for a measure that is linked to his own welfare, presumably the utility of the richest. It may be clear that these two examples are two opposites and result in a different ordering of possible transactions. In this thesis we use the social welfare function that is just the sum of the individual utilities, this is also called the utilitarian social welfare function. Moreover the corresponding function that selects the most preferred transaction is called the utilitarian social choice function. (Note that from an ethical point of view this might be an immoral choice if we were talking about the allocation of the primary necessities of life. However, in this thesis we are considering the production costs of companies, meaning that this concept corresponds to choosing the solution with lowest total costs.)

Another concept is efficiency. Efficiency may have different meanings. One meaning relates to production. In this setting input is used to produce output. Production is called efficient if both

- one can not produce more output using the same amount of input, and

- one can not produce the same output using less input.

More generally, one can regard all production costs as 'input', giving the usual efficiency concept in optimization, producing at lowest cost or highest profit. Another meaning of efficiency relates to the allocation of goods over different consumers. The corresponding efficiency concept is called Pareto efficiency (or Pareto optimality). An allocation is 
called (Pareto) efficient if there is no other allocation that gives all consumers at least the same utility where at least one of them has a strictly higher utility.

In this thesis these concepts of efficiency overlap. We are aiming for production efficiency for a supply chain as a whole, so for a set of producers. However, production efficiency relates to the costs of the suppliers, more precisely, production is efficient if demand is produced at the lowest possible costs. At the same time it holds that the sum of the utilities of the suppliers (i.e. social welfare) is maximized if the total costs are minimized. Consequently we know that this solution is Pareto efficient as otherwise there is a contradiction with maximal social welfare.

A game is a situation in which for each individual in some group, individual utility depends on both its own action and the others' actions. So, in general, the best action to choose depends on the action chosen by the others. Mas-Colell et al. [31] list the following four elements that are needed to describe a game:

- Players: the individuals involved.

- Rules: the actions that the players may take.

- Outcomes: the reallocation of goods and money for each possible combination of actions by the players.

- Payoffs: the preferences, i.e. the utility functions, of the players over the possible outcomes.

A standard example of a game is the well known prisoner's dilemma. In this game with two players it is clear for both players what action to choose. Namely, one action is the best reaction on every possible action of the other player. However, the resulting outcome is not efficient as there is another outcome that is better for both players.

Example 1.3.1 (Prisoner's dilemma) Consider a game of two players. The players are $B$. and $V$., two prisoners that are arrested for allegedly being involved in murdering their former friend. The prisoners are kept in separate cells and both can choose between confessing that they committed the crime together and deny that they were involved in the crime. The prosecutor can only prove wilful murder for both of them if at least one of the prisoners confesses, otherwise only the less severe crime of manslaughter can be proven. In fact, if both deny, they will both be in prison for seven years. A prisoner that denies the crime, while wilful murder can be proven will be sentenced in jail for fifteen years, while confessing the crime reduces the sentence to nine years. The prosecutor offers both prisoners that they get an additional reduction in sentence of four years if they are the only prisoner to confess, 
i.e. if they are essential for proving wilful murder. The choices are summarized in Table 1.1. If $B$. knows that $V$. denies, he has a choice between 7 and 5 years of jail for respectively

\begin{tabular}{l|cc} 
& V. Denies & V. Confesses \\
\hline B. Denies & 7,7 & 15,5 \\
B. Confesses & 5,15 & 9,9
\end{tabular}

Table 1.1: Years of jail for B. and V.

denying and confessing. So B. prefers confessing. Otherwise, if he knows that $V$. confesses the choice is between 15 and 9, and again confessing is the preferred option. Similarly, the same holds for $V$. if he knows the choice of $B$. We conclude that for both prisoners it is best to confess, giving both of them nine years of jail. Ironically, if they could both have agreed on denying, they would only be in prison for seven years.

The situation 'B. confesses, V. confesses' in Example 1.3.1 is called an equilibrium. An equilibrium is a combination of actions for all players such that it holds for each player that given the other players' actions, the player won't change its own action. Furthermore, 'confess' is for both players a dominant strategy. A dominant strategy is an action such that no matter what the other players' actions are, the action is strictly preferred over all the other actions. If an action is only at least as good as any other action, it is called a weakly dominant strategy.

Games exist in several formats and complexities, see for example Fudenberg and Tirole [15], Mas-Colell et al. [31] and Montet and Serra [32]. Cachon and Netessine [6] give an overview of applications in supply chain analysis. In section 1.3.1 we discuss auctions, games that are designed to sell goods or, in reverse form, to buy goods. This is the type of game that we consider in this thesis. Auctions are not only special kinds of games, but also special kinds of mechanisms, i.e. games that are especially designed to achieve some goal. In section 1.3.2 the field of mechanism design will be discussed.

\subsubsection{Auctions}

An auction is a public sale where prices are not fixed in advance, but depend on the bids of potential buyers. A simple and useful auction format is the English auction. English auctions are a very common way to sell art and antiques, for example at the famous Christie's auction house in London, UK. The format is very easy: a single good is offered for some minimum price and bidders may give new bids as long as they bid more than 
the current highest bid. The highest bidder gets the good and has to pay his or her latest bid price. To get the good you have to bid (marginally) more than the highest valuation from the other bidders. For example, if there are three other bidders, estimating the value of the good on 7, 8 and 9 euros respectively, you have to bid at least 9 euros to get the good. As you would only do that if your own valuation is more than 9 , the price you have to pay is (approximately) the second highest valuation from the whole set of bidders.

Another auction format is the Dutch or descending clock auction. This kind of auction is commonly used for the selling of agricultural products like flowers and vegetables, for example at the Aalsmeer Flower Auction in The Netherlands. This auction works the other way around as the English auction: it starts at a high price, where the auctioneer lowers the price until a bidder is willing to pay that price. Like in the English auction, the bidder will pay exactly his own bid price. The English auction and the Dutch auction are called ascending and descending respectively according to the price development during the auction.

The English auction is related to the Vickrey or second-price sealed-bid auction, named after Vickrey [52]. The Vickrey auction concerns the sale of a single indivisible good. Bidders are asked for (sealed) bids, and the highest bidder will receive the good at the second highest bid price. No matter the valuations or bidding strategies of the other bidders, it is optimal for a bidder to bid its true valuation for the item. Due to this property, the final transaction in the Vickrey auction is the same as in the English auction.

In this thesis, we use an auction mechanism that is closely related to the Vickrey auction and has the same incentive characteristics, i.e. for all participants truthtelling is a (weakly) dominant strategy. This means that bidding the true valuation is one of the preferred actions for all bidders. Vickrey [52] himself generalized the Vickrey auction to the case in which multiple homogeneous goods are sold, and each bidder still wants only one of them. In this case the highest bidders receive the goods and the unit price will be equal to the first rejected bid. We investigate the further generalization to multiple heterogeneous goods where bidders may be interested in combinations of different goods. Moreover, we assume that the valuation of one good may depend on the possession of another good. For example, having a mobile phone may only be valuable if you also have a SIM card from a mobile operator. We say that the phone and the SIM card are complements. Goods can also be substitutes: one might consider buying a Nokia mobile phone or a Samsung, but not both.

Auctions in which bidders can bid on combinations of goods are called combinatorial 
auctions. These auctions have recently been discussed in Cramton et al. [8]. One of the issues is how to design an appropriate (combinatorial) auction for a specific setting. See De Vries and Vohra [54] for a review on this issue. Auction design relates to the field of mechanism design, which we discuss next.

\subsubsection{Mechanism design}

If you want to sell something you have to choose how to do this, as there may be several options to organize the selling. For example, you can have bilateral negotiations, or you can perform an auction like the ones described in the previous section. The best way to sell depends on the goals you have in mind. Pekeč and Rothkopf [40] distinguish for example revenue maximization, short running time of the selling process, and low transaction costs. Auctions are a widely applied way to sell goods, or in reverse form to buy goods. The rise of the internet enabled many applications as auctions can be run very efficiently on the internet, see for example Hohner et al. [24]. Still, there is a virtually infinite number of ways to organize an auction, so you have to design the rules of the auction such that it has the properties you like. As there is no one auction format that fits all possible needs perfectly, auctions should be designed for specific needs and priorities. This is called auction design, which is a special case of the more general field of mechanism design.

Mechanism design is the field of research that focusses on the design of games to achieve a specified outcome by giving all players involved an incentive to act such that this outcome is achieved. For example, the English auction is a game that is designed to sell a good to the bidder with the highest valuation for it. Mechanism design is a branch of game theory that not only analyzes strategic interaction between players but also actively shapes the situation itself:

"Rather than formulate a game that captures an economic situation and look for the properties of the likely outcome given by some equilibria, we are here fixing a set of outcomes satisfying some desirable properties and look for a game whose equilibria yield that outcome." Montet and Serra [32, page 49]

So, a mechanism is a game that is designed in such a way that its equilibrium yields the outcome wanted by the mechanism designer. A mechanism in which the players have an incentive to behave in the desired way is called incentive compatible, which is a necessary property for being an appropriate mechanism.

Usually in mechanism design it can be assumed that the only action of the players of 
the game is to reveal their preferences to a neutral mechanism designer. These mechanisms are called direct revelation mechanisms. The idea behind this assumption is that the mechanism designer will play the game for all players according to their preferences and the rules of the game. This idea is called the revelation principle, see for example Mas-Colell et al. [31]. Mas-Colell et al. [31] distinguish two situations in which the revelation principle can be applied. First, the revelation principle can be applied to situations in which all players have a weakly dominant strategy. Second, it applies to situations in which there is a Bayes-Nash equilibrium, i.e. the equilibrium action of each player is in expectation the best response to the equilibrium actions of the other players. In the first situation it is completely clear for the mechanism designer what the action of the players would be. The second situation might have more than one equilibrium, in which the mechanism designer has to choose the appropriate one, as not all of the equilibria might lead to the specified outcome, see Mas-Colell et al. [31, page 910-912]. In both situations where the revelation principle can be applied, the players have an incentive to report their true preferences, as then the mechanism will select their preferred action. So, in direct revelation mechanisms, incentive compatibility corresponds to truthful reporting the individual preferences.

Using the revelation principle, direct revelation mechanisms consist of two phases:

- Phase one: all players reveal their preferences.

- Phase two: the mechanism designer plays the game, and implements the outcome of the game, i.e. goods and money are transferred giving the players their payoffs.

A mechanism is in fact a screening game:

"In a screening game the player that lacks information is the first to move." (Cachon and Netessine [6])

In this terminology the mechanism designer is the player who moves first by designing a game for the others that gives them the incentive to reveal their preferences.

Before we can continue with some desirable characteristics of mechanisms and one specific mechanism, we need the following formalization. Let $A$ be a set of players, and let $t_{a}$ denote the preferences of player $a \in A$. We call $t_{a}$ the type of player $a$. The types of the players are assumed to be private information, but it is publicly known which values they may possibly have. Therefore, we denote by $T_{a}$ the type space of player $a \in A$, i.e. the set of types that player $a$ can possibly have. Write $T \equiv \prod_{a \in A} T_{a}$ for the subspace of types of all players, with $t=\left(t_{a}\right)_{a \in A} \in T$. Let $O$ be the set of outcomes, i.e. the possible 
reallocations of goods and money. By $v_{a}\left(t_{a}, o\right)$ we denote the valuation that player $a$ assigns to outcome $o$ when being of type $t_{a}$. Players are assumed to have quasi-linear utilities: the net valuation (or payoff) for outcome $o$ and paying $p_{a}$ by player $a$ being of type $t_{a}$ is given by $v_{a}\left(t_{a}, o\right)-p_{a}$. Here, a negative payment means that the player receives money.

Recall that in the first phase of the mechanism, the players reveal their preferences, and in the second phase the mechanism determines the outcome and the payments as functions of the revealed preferences. Therefore, we may write $o(t)$ and $p_{a}(t)$ for the outcome and the payment of player $a$ that are determined by the mechanism.

\subsubsection{Desirable characteristics}

As said before, a mechanism is a properly designed game whose equilibrium yields a desired outcome. For example, the desired outcome may be to implement a given social choice function. Recall from section 1.3 that a social choice function selects the transaction or outcome that maximizes social welfare, depending on the types of the players. Formally, let $\omega$ denote the social choice function, which selects outcome $\omega(t)$ in $O$ when $t=\left(t_{a}\right)_{a \in A}$, i.e. $\omega: T \rightarrow O$.

We say that a mechanism implements the social choice function if its equilibrium yields the same outcome as $\omega(t)$. Apart from being an incentive compatible direct revelation mechanism, the mechanism should have some other characteristics for being implementable in many practical situations. We discuss individual rationality, efficiency and budget balance.

Next to incentive compatibility, i.e. the property that players act in the desired way, we need to give the players an incentive to play the game in the first place. As in general we cannot force players to participate, they will only do so if it is profitable. This means that a mechanism should guarantee non-negative utilities to players. An outcome $o(t)$ together with payments $\left(p_{a}(t)\right)_{a \in A}$ is called individual rational if $u_{a}(t) \equiv$ $v_{a}\left(t_{a}, o(t)\right)-p_{a}(t) \geq 0$ for all $a \in A$. Moreover a mechanism is called individual rational if for any type profile $t$ we have that $u_{a}(t) \geq 0$ for all $a \in A$.

Another desirable characteristic is efficiency, which we introduced at the beginning of section 1.3. Here we relate the concept of efficiency to the utilitarian social welfare. Write

$$
V(A, t) \equiv \max _{o \in O} \sum_{a \in A} v_{a}\left(t_{a}, o\right)
$$

for the maximal total valuation of all players, given their preferences $t$. Then, an out- 
come $o$ is called efficient if

$$
\sum_{a \in A} v_{a}\left(t_{a}, o\right)=V(A, t)
$$

Furthermore, a mechanism is called efficient if for any possible type profile of the players it leads to an efficient outcome. Using this terminology, we can say that a social choice function $\omega$ is utilitarian if $\omega(t)$ is an efficient outcome for any profile $t=\left(t_{a}\right)_{a \in A}$ of types.

Part of a mechanism is the payment of the players. In cases where the mechanism itself has no money to pay to the players, which is usually true, the mechanism should be (weakly) budget balanced. Recall that $p_{a}(t)$ is the amount paid by player $a$ if type profile $t$ is reported. The total payment of the players is called (weakly) budget balanced if $\sum_{a \in A} p_{a}(t) \geq 0$. Moreover the mechanism is called (weakly) budget balanced if for any possible type profile of the players the payments are (weakly) budget balanced.

It is possible for some problem classes to design an incentive compatible mechanism that is efficient, (weakly) budget balanced and individually rational for any problem instance. An example of this is the Vickrey auction for selling an individual good, see Vickrey [52] and section 1.3.1. It is efficient as it assigns the good to the player with the highest valuation. It is budget balanced as the winner pays money to the auctioneer while the other players do not pay or receive money. Finally it is individual rational as the winner pays less than its own bid, while the other players have zero utilities. The fact that the Vickrey auction has all three characteristics relates to the fact that none of the bidders is essential in the sense that if one bidder leaves, another can buy the item. So, none of the bidders has that much power to block any transaction. However, in exchanges or bilateral trade settings players can become mutually dependent for generating profit. Myerson and Satterthwaite [33] proved a well known impossibility result for the bilateral trade setting. In their case it becomes impossible to design incentive compatible mechanisms that achieve efficiency, budget balance and individual rationality at the same time. This impossibility result is also troubling the supply chain setting in which a buyer and a supplier can only improve the uncoordinated planning if they both agree. The impossibility result holds for several other settings, see Mas-Colell et al. [31, p. 493] and Montet and Serra [32] and the references therein.

\subsubsection{VCG mechanism}

One well-known mechanism is the VCG mechanism, named after Vickrey [52], Clarke [7] and Groves [18], and is essentially a generalization of the Vickrey auction, which we discussed in section 1.3.1. Groves [18] presents the most general model, based on the theory of teams. 
"A team decision problem is, roughly speaking, a multi-person joint decision problem in which the decision makers base their decision choices on different information, yet are motivated by a common goal." Groves [18, page 618]

The setting of Groves is that of an organization, in which the organization's leader and some employees are decision makers. The problem is that the employees may not have the same goals as the organization's leader. To handle this, a model is built in which the leader pays money to the employees to incite them to act in line with the organization's goal. A more specific model is the one of Clarke [7]. Mas-Colell et al. [31] say the following about the relation between the work of Groves [18] and the work of Clarke [7]:

"A special case of the Groves mechanism was discovered independently by Clarke (1971) and is known as the Clarke, or pivotal, mechanism."

In fact this is the basic form of what is called the VCG mechanism. The VCG mechanism is a direct revelation mechanism that implements the utilitarian social choice function, i.e. it chooses the allocation that maximizes the total utility. Like in the Vickrey auction, it is a dominant strategy for all players to report truthfully, i.e. no matter what the reports of the other players are it is best to report truthfully.

Let $\omega$ be the utilitarian social choice function that we want to implement by a mechanism. Recall that $t=\left(t_{a}\right)_{a \in A}$ is the profile of types of the players. Furthermore, let $t^{-a}=\left(t_{b}\right)_{b \in A \backslash\{a\}}$ denote the profile of types of all players except $a$. We can now specify the VCG mechanism, where we already assume that all players report truthfully. Later on we show that this assumption is correct. Given the (truthful) reports of the players, the VCG mechanism chooses the outcome $\omega(t)$. Payments are defined as follows. The payment that player $a$ has to make is given by

$$
p_{a}(t) \equiv v_{a}\left(t_{a}, \omega(t)\right)+V\left(A \backslash\{a\}, t^{-a}\right)-V(A, t),
$$

which is known as the Vickrey payment. (The Groves mechanism replaces $V\left(A \backslash\{a\}, t^{-a}\right)$ by a general function $h_{a}\left(t^{-a}\right)$, having the same incentive characteristics. The Vickrey payment is exactly such that the VCG mechanism applied to the auctioning of one item is the same as the Vickrey auction.) The resulting net valuation (or payoff) of player $a$ is equal to its marginal contribution or marginal product

$$
v_{a}\left(t_{a}, \omega(t)\right)-p_{a}(t)=V(A, t)-V\left(A \backslash\{a\}, t^{-a}\right) .
$$


The VCG mechanism has several nice properties, see Mas-Colell et al. [31]. Most importantly, reporting truthfully is a (weakly) dominant strategy, i.e. the VCG mechanism is an incentive compatible direct revelation mechanism. It is easy to see that reporting the true preferences or type is a dominant strategy as we present in the following proposition.

Proposition 1.3.2 Reporting the true valuations is a dominant strategy in the VCG mechanism.

\section{Proof:}

If player $a$ chooses to report $r_{a}$ instead of $t_{a}$, and the other players report some arbitrary $r^{-a}$, player $a$ has payoff equal to

$$
V(A, r)-V\left(A \backslash\{a\}, r^{-a}\right)+v_{a}\left(t_{a}, \omega(r)\right)-v_{a}\left(r_{a}, \omega(r)\right)
$$

while reporting truthfully $t_{a}$ gives a payoff equal to

$$
V\left(A,\left(t_{a}, r^{-a}\right)\right)-V\left(A \backslash\{a\}, r^{-a}\right)
$$

So the gains from reporting $r_{a}$ instead of $t_{a}$ are the difference between the payoffs above:

$$
V(A, r)-V\left(A,\left(t_{a}, r^{-a}\right)\right)+v_{a}\left(t_{a}, \omega(r)\right)-v_{a}\left(r_{a}, \omega(r)\right) .
$$

Player $a$ wants to choose $r_{a}$ such that these gains are maximized. Note that $V\left(A,\left(t_{a}, r^{-a}\right)\right)$ is independent from $r_{a}$, so we can restrict our attention to the exprssion

$$
v_{a}\left(t_{a}, \omega(r)\right)+\left(V(A, r)-v_{a}\left(r_{a}, \omega(r)\right)\right) .
$$

The first term of this expression is the (true) valuation of player $a$, while $(V(A, r)-$ $\left.v_{a}\left(r_{a}, \omega(r)\right)\right)$ is the (reported) valuation of all the other players. In total, this is exactly what is maximized by the mechanism if player $a$ reports truthfully. Consequently, the gains of player $a$ are maximized if he reports truthfully.

In chapters 2, 3 and 4 we use the VCG mechanism for a combinatorial procurement auction, where the valuations of the bidders correspond to costs, i.e. their valuations are negative. So, knowing that the players will tell the truth and the mechanism chooses the cost-minimizing solution, player $a \in A$ receives a VCG-payment of

$$
C_{A}^{a}+\left(C_{A \backslash\{a\}}-C_{A}\right),
$$


where $C_{A}^{a}$ denotes the cost faced by player $a$ in the optimal allocation, and $C_{A}$ denotes the total costs of the optimal allocation for the set of players $A$. In total, the auctioneer pays

$$
\sum_{a \in A}\left[C_{A}^{a}+\left(C_{A \backslash\{a\}}-C_{A}\right)\right]=C_{A}+\sum_{a \in A}\left(C_{A \backslash\{a\}}-C_{A}\right) .
$$

Observe that the marginal product of any bidder that does not win equals zero. As a consequence the payment reduces to $C_{A \backslash\left\{a_{1}\right\}}$ if there is only one winner $a_{1}$. So, indeed, if only one good is auctioned the VCG mechanism is equivalent to a second-price or Vickrey auction [52].

\subsection{Coordination in supply chain planning: motivating examples}

In section 1.2 we presented two models for production planning, a continuous model with static data, and a discrete model with dynamic data. These two models are the basic point of view from which we consider coordination in supply chains.

First, Dudek and Stadtler [9] consider the coordination of buyers and suppliers in the discrete dynamic setting. They propose a negotiation scheme that enables the supply chain partners to come close to the optimal coordinated solution. However, one assumption is that all partners voluntarily provide private information, i.e. information that cannot be verified by the other party. We show that it can be very expensive to achieve incentive compatibility in this setting. As a solution we introduce supplier competition in the form of an auction. In chapters 2,3 and 4 the performance of this auction will be assessed and compared with auctions that do not take into account the lot-sizing cost structure of suppliers. The proposed auction uses the VCG mechanism, which has nice theoretical properties, but might be hard to implement. In chapter 5 we discuss the VCG mechanism for a very basic problem. For this problem it can be shown that some nice properties enable the use of the VCG mechanism. We also show that these properties do not hold for some rather straightforward extensions of the problem. This implies that an English auction-like ascending price implementation of the auction we propose in chapter 2 might not achieve the theoretical optimal solution.

Second, we discuss the work of Sucky [49] who introduces the problem of asymmetric information in the case where the EOQ problems of a buyer and a supplier are combined in one problem. Sucky discusses how the supplier can determine a set of offers to the buyer that fulfills conditions of individual rationality and incentive com- 
patibility. The offers can be determined analytically in the EOQ setting, but the related problem in single-item lot-sizing yields a mathematical programming problem. A basic version of this problem will be discussed in chapter 6 .

\subsubsection{Negotiation-based planning in supply chains}

Dudek and Stadtler [9] consider a generalization of the lot-sizing problem (LSP) over different echelons of a supply chain, where the different lot-sizing problems are linked to each other. In this setting a buyer needs production input from a supplier, similar to the setting of Sucky [49], but here they consider the discrete lot-sizing problem instead of the continuous EOQ model. The problem can be modeled as a generalization of (LSP), called the multi-level lot-sizing problem (MLSP). However, it is not always possible to optimize (MLSP), especially if the different echelons of the supply chain belong to different companies. In such a situation, firms may fear sharing information (see Griffin and Scherrer [17]), making a joint planning impossible.

Dudek and Stadtler [9] propose a negotiation based scheme to coordinate the planning over the different echelons, without sharing all information. In the following we present their scheme for a two-player case trading one product, although their models can be applied to more general supply chain structures with more players and more products.

Suppose we have a supplier and a buyer, each with a production planning problem of the form (LSP). Assume that the demand of the supplier is given by the production requirements of the buyer, where we assume that the buyer needs one unit of input for each unit of output. We index the variables of the buyer and supplier by $b$ and $s$ respectively. From this we get the following multi-level lot-sizing problem:

$$
\min \sum_{t=1}^{T} \sum_{j \in\{b, s\}}\left(p_{t}^{j} x_{t}^{j}+h_{t}^{j} I_{t}^{j}+f_{t}^{j} y_{t}^{j}\right)
$$

subject to:

$$
\begin{aligned}
I_{t-1}^{j}+x_{t}^{j} & =d_{t}^{j}+I_{t}^{j}, \quad \forall t \in\{1, \ldots, T\}, \forall j \in\{b, s\}, \\
d_{t}^{s} & =x_{t}^{b}, \quad \forall t \in\{1, \ldots, T\}, \\
x_{t}^{j} & \leq C_{t}^{j} y_{t}^{j}, \quad \forall t \in\{1, \ldots, T\}, \forall j \in\{b, s\}, \\
I_{0}^{j} & =0, \quad \forall j \in\{b, s\}, \\
I_{t}^{j} & \geq 0, \quad \forall t \in\{1, \ldots, T\}, \forall j \in\{b, s\}, \\
x_{t}^{j} & \geq 0, \quad \forall t \in\{1, \ldots, T\}, \forall j \in\{b, s\},
\end{aligned}
$$




$$
y_{t}^{j} \in\{0,1\}, \quad \forall t \in\{1, \ldots, T\}, \forall j \in\{b, s\} .
$$

Constraints (1.8) link the production planning of the buyer and the supplier. In the negotiation scheme of Dudek and Stadtler [9] this link is subject to negotiations, and demand for the supplier is not directly fixed by the production quantities of the buyer.

The negotiation process runs as follows.

A first step is that an upstream planning is determined. This means that the buyer solves its own single-level lot-sizing problem, without taking into account the capacities and cost structure of the supplier. For being able to implement this plan, some input vector is needed from the supplier. Given this vector the supplier solves its own local lot-sizing problem, minimizing the costs to produce the order of the buyer. (Here Dudek and Stadtler [9] assume that capacity restrictions are flexible in the sense that additional capacity can be hired at some higher cost. As this is beyond our focus, we omit the corresponding adaptation of problem (MLSP).)

The second step allows the supplier to change the proposals of the buyer. As a maximum the supplier can shift all demands to the next or previous period with a positive demand, as long as the resulting demand pattern is feasible to the buyer. So, in fact the supplier faces a generalization of the lot-sizing problem in which the demand vector can be adapted marginally. The resulting solution is proposed to the buyer. These first two steps initialize the negotiation process. The next two steps are repeated until some condition holds.

In the third step it is the turn of the buyer. The buyer first determines the costs of two extreme solutions:

- The minimal costs if the proposed solution is accepted, and

- the minimal costs if the proposed solution can be adapted within the same bounds as faced by the supplier in the previous step.

Naturally, the second solution is the best one for the buyer, being the same as its latest proposal to the supplier. However, it is assumed that the buyer does not choose its own personal best, but determines a balanced solution between the two extremes given above. The idea is to only propose the most effective changes and leave changes that only give a minor cost reduction. The buyer sends this proposal to the supplier together with its increase in costs compared to the upstream solution. This enables the supplier to judge its own cost reduction against the cost increase of the buyer.

In the next step it is the supplier's turn again. The supplier first determines the cost if the same type of shifts were allowed as in the second step. But now, like the buyer 
did in the previous step, the supplier will not propose this personal best. Instead the supplier weighs its own savings and the estimated cost increase of the buyer. And again the resulting plan is sent to the buyer, repeating the previous step et cetera.

The process finishes at some point by using a simulated annealing criterium. Dudek and Stadtler [9] say that computational tests suggest that this negotiation scheme comes close to optimal results as obtained by central coordination, i.e. solving the multi-level lot-sizing problem at once. On average the test results show a cost difference of only $1.6 \%$.

The approach of Dudek and Stadtler [9] is very promising in situations where we may assume that the players (i.e. the buyer and supplier) will report truthfully their abilities and their cost changes. One may think of situations within one company where no central planning authority exists, but where all parts are interested in the company's total profit. However, in situations where the supply chain is formed by independent companies, which is the focus of this thesis, the assumption of truthful reports will not hold. It is an important question how independent players may be able to negotiate a solution that is good enough for being a competitive supply chain.

Therefore, in the following chapters, we focus on the same underlying setting as Dudek and Stadtler [9] together with the assumption that the buyer and supplier behave selfishly. This means that we have to apply some mechanism such that the individual goals are made incentive compatible, i.e. acting selfishly is compatible with acting in the interest of the supply chain. A first intuition is to apply the VCG mechanism due to its nice (theoretical) properties. However, in the next example we see that this mechanism is far from budget balance, where we have no third party that is willing to pay for the budget deficit.

Example 1.4.1 Consider a supply chain with one buyer $b$ and one supplier s, facing lotsizing problem (MLSP). The buyer has the market power to impose its optimal lot-size policy, giving the upstream planning solution. Assume that both the buyer and the supplier do not know each other's cost profile. Now we apply the VCG mechanism to get to the optimal solution of the chain's optimization problem, using the notation as introduced in section 1.3.2. Let $V(A, t)$ denote the maximal cost reduction that can be achieved compared to the upstream plan, where $A=\{b, s\}$. Furthermore, if either of the players does not cooperate, no cost reduction can be achieved. Therefore it holds that $V\left(A \backslash\{b\}, t^{-b}\right)=$ $V\left(A \backslash\{s\}, t^{-s}\right)=0$. The VCG payment of the buyer is:

$$
p_{b}(t)=V\left(A \backslash\{b\}, t^{-b}\right)-V(A, t)+v_{b}\left(t_{b}, \omega(t)\right)=v_{b}\left(t_{b}, \omega(t)\right)-V(A, t) .
$$


The VCG payment of the supplier is:

$$
p_{s}(t)=V\left(A \backslash\{s\}, t^{-s}\right)-V(A, t)+v_{s}\left(t_{s}, \omega(t)\right)=v_{s}\left(t_{s}, \omega(t)\right)-V(A, t) .
$$

The utility of both the buyer and the supplier is therefore equal to $V(A, t)$, i.e. both players receive the whole cost reduction that was achieved. This means that the cost reduction itself is insufficient to pay both players, giving a budget deficit: $p_{b}(t)+p_{s}(t)=-V(A, t)$.

To overcome this budget difficulty, we introduce competition in this setting. Like before we assume that there is only one buyer facing a multi-period problem. But now we assume that the buyer can get its input from different suppliers. Furthermore, the choice between these suppliers will be made by using a procurement auction mechanism. In the next chapter this mechanism will be introduced.

\subsubsection{A bargaining model with asymmetric information}

In this section we present a bargaining model with asymmetric information for a single supplier-single buyer problem, as described by Sucky [49]. The setting is a supply chain with one buyer $b$ and one supplier $s$, facing a continuous demand rate $d$ at the buyer level, and a production rate at the supplier level of $m$. This basic setting was introduced by Banerjee [2]. First consider the buyer. The buyer faces an economic lot-size problem like we presented in section 1.2.1, having inventory holding costs $h_{b}$ and fixed ordering costs $f_{b}$. The cost minimizing economic ordering quantity $Q_{b}^{*}$ and the corresponding per period costs $K_{b}\left(Q_{b}^{*}\right)$ are respectively

- $Q_{b}^{*}=\sqrt{\frac{2 f_{b} d}{h_{b}}}$, and

- $K_{b}\left(Q_{b}^{*}\right)=\sqrt{2 f_{b} d h_{b}}$.

Furthermore, the supplier faces a production rate $m>d$, meaning that uninterrupted production results in overproduction. So, to fulfill demand the supplier faces alternately periods of production and periods of idle time. Similarly to the EOQ model, Sucky [49] recalls the following cost minimizing formulas for the supplier's economic lot-size $Q_{s}^{*}$ and per period costs $K_{s}\left(Q_{s}^{*}\right)$ respectively, having inventory holding costs $h_{s}$ and fixed production setup costs $f_{s}$,

- $Q_{s}^{*}=\sqrt{\frac{2 f_{s} m}{h_{s}}}$, and

- $K_{s}\left(Q_{s}^{*}\right)=d \sqrt{\frac{2 f_{s} h_{s}}{m}}$. 
Here it is assumed that every time that the supplier finishes a complete lot, this is shipped to the buyer immediately. However, buyer and supplier can not just choose their own optimal schedule as the optimal supply schedule may be different from the optimal ordering schedule. Minimizing the total costs of supplier and buyer together, gives the following optimal integrated production and ordering policy, where $Q_{J}^{*}$ is the joint optimal lot-size and $K_{J}\left(Q_{J}^{*}\right)$ the joint optimal per period costs,

- $Q_{J}^{*}=\sqrt{\frac{2 d\left(f_{b}+f_{s}\right)}{h_{b}+\frac{d}{m} h_{s}}}$, and

- $K_{J}\left(Q_{J}^{*}\right)=\sqrt{2 d\left(f_{b}+f_{s}\right)\left(h_{b}+\frac{d}{m} h_{s}\right)}$.

The question is whether the buyer and supplier would be able to agree on this solution. Sucky [49] says:

"If $b$ and $s$ behave individually rational, they select their individual optimal policies $Q_{b}^{*}$ and $Q_{s}^{*}$. If $b$ or $s$ has market power to impose its individual optimal policy on $s$ or $b$, respectively, then no incentive exists for either $b$ or $s$ to deviate from their individual optimal policy $Q_{b}^{*}$ or $Q_{s}^{*}$. The weaker player must deviate from his individual optimal policy and adapt to the stronger players policy. However if negotiations are possible, the weaker player may try to persuade the stronger player to select a policy other than its individual optimal policy by a side payment." (The quote is adapted to the notation of this thesis.)

Sucky [49] shows that in case of complete information (i.e. players do not have to reveal private information), the bargaining solution will be the same as the joint optimal solution.

Things get much more complicated if players do not have complete information. Therefore, Sucky [49] studies the case in which the buyer has the market power to impose its optimal lot-size policy, while the supplier does not know the cost structure of the buyer. The idea is that the supplier can offer a combination $(Q, p)$ to the buyer, where $Q$ is the proposed lot-size and $p$ the compensation that the supplier pays to the buyer to change its own optimal policy. However, the optimal bid depends on the true cost structure (called type) of the buyer. Let $N \equiv\{1, \ldots, n\}$ be the set of possible types of the buyer, where type $i \in N$ is assumed to occur with probability $\lambda_{i}$. Then the supplier may offer different bids tailored to the different types, as long as incentive compatibility and individual rationality can be assured, i.e.

- a buyer of type $i$ prefers the bid made for type $i$ (incentive compatibility), and 
- choosing this bid is preferred over $\left(Q_{i}^{*}, 0\right)$, where $Q_{i}^{*}$ is the individual optimal lotsize for the buyer of type $i$ (individual rationality).

This game is a screening game (see section 1.3.2) as the uninformed player designs a menu of choices for the informed player who indirectly reveals its type by choosing the preferred bid. The screening problem of the supplier can be formalized in the following mathematical program, where $\left(Q_{i}, p_{i}\right)$ is the bid designed for buyer type $i, K_{s}(\cdot)$ is the cost function of the supplier, and $K_{i}(\cdot)$ is the cost function of a buyer of type $i$ :

$$
\min _{Q, p} \sum_{i=1}^{n} \lambda_{i}\left(K_{s}\left(Q_{i}\right)+p_{i}\right)
$$

subject to:

$$
\begin{aligned}
K_{i}\left(Q_{i}\right)-p_{i} & \leq K_{i}\left(Q_{i}^{*}\right), \quad \forall i \in N, \\
K_{i}\left(Q_{i}\right)-p_{i} & \leq K_{i}\left(Q_{j}\right)-p_{j}, \quad \forall i, j \in N, \\
Q_{i} & >0, \quad \forall i \in N \\
p_{i} & \geq 0, \quad \forall i \in N .
\end{aligned}
$$

Sucky [49] is able to derive analytically a set of possible solutions that fulfill the KarushKuhn-Tucker conditions. An optimal solution of a problem instance can then be found by calculation the costs for each of these solutions.

A similar formulation can be given for the the case of a discrete and finite timehorizon. Then, unlike the continuous setting of Sucky [49], the preferred planning of each of the players can not be expressed by a single number. Instead they will have a preferred supply vector that should fulfill the constraint that it is sufficient to deliver the demand at the buyer level. Although the set of feasible supply vectors can be assumed to be finite (using the zero-inventory property of Wagner and Whitin [55]), finding an optimal solution to this problem is much less trivial than for the continuous problem. As we can not give explicit cost functions $K_{s}(\cdot)$ and $K_{i}(\cdot)$, we have to find an optimal solution by enumeration of all possible solutions. Moreover, the number of solutions is exponential in both the number of types and the number of periods. We conclude that unlike the relatively easy method of Sucky [49] for the continuous problem, as yet the discrete setting can not be solved efficiently.

Problem (SG) has a feasible region that is defined by incentive compatibility and individual rationality constraints. The same structure of constraints may appear in the design of incentive compatible and individual rational auctions, see section 1.3.2.1. The corresponding optimization problems are already nontrivial for very simple cost functions. In chapter 6 we describe one of those problems, called the parametric shortest 
path tree problem. This is a generalization of the shortest path tree problem where we have to choose parameter values of the arc-length functions. The arc length functions are given by arc-dependent constants and node-dependent variables (the parameters to choose). We show that an optimality condition holds that is related to a local improvement algorithm, but this algorithm itself may need an exponential or even infinite number of steps.

\subsection{Outline of the thesis}

The rest of the thesis is organized as follows. In chapter 2 we introduce a combinatorial auction for a multi-period procurement setting. In this setting there is one buyer who organizes a procurement auction for a group of possible suppliers. Although we consider only one type of good, the multi-period character of the problem causes the combinatorial character. More specifically, the dynamic costs (especially setup costs) and capacities corresponding to the lot-sizing problems of suppliers give the costs a nonlinear character, which is shown in section 2.2. Even though the combinatorial auction allows bidders to express their costs appropriately in their bids, it might be better for the auctioneer to use less sophisticated auction mechanisms. In section 2.3 we present two alternative auction mechanisms that have a strong relation to the extreme solutions of the lot-sizing problem. A first alternative is to have one auction that procures all demand in the first period, leaving the auctioneer with the highest possible inventories. As this auction builds up stock for the entire planning horizon already in the first period, we call this the stock auction. The second alternative is to organize an auction in each period separately and preclude the possibility of inventories. We have the counterintuitive result that either of the alternative auction formats, that do limit production flexibility, might be cheaper for the auctioneer than the combinatorial procurement auction. However, we also show that for the two-period case, the combinatorial auction seems to be an attractive compromise between the alternatives as it is never the most expensive of the three.

Chapter 3 compares the procurement costs of the combinatorial auction and the alternative of organizing an auction in each period separately. In section 3.2 we show that if suppliers are not limited by capacities, the combinatorial auction dominates the separate auctions as it is never more expensive for the auctioneer than the separate auctions. For capacitated problems the separate auctions might be the cheapest solution for the auctioneer for some special cases. However, in section 3.3 we show for a two-

period scenario that in expectation the combinatorial auction dominates the separate 
auctions.

Chapter 4 compares the procurement costs of the combinatorial auction and the stock auction. In section 4.2 we show that if suppliers are not limited by capacities, the combinatorial auction dominates the stock auction as it is never more expensive for the auctioneer than the stock auction. On the contrary, for capacitated problems the stock auction might be the cheapest option for the auctioneer. However, in a twoperiod scenario, the possible loss from using the combinatorial auction instead of the stock auction can be limited in two ways. First, this loss is limited by the setup costs. Second, we show that the loss depends on the level of competition between suppliers. More specifically, the loss is limited by the difference in unit production costs of the two cheapest suppliers in the second period.

In chapter 5 we have a closer look at the VCG mechanism that we used in the previous chapters. This mechanism has some nice theoretical advantages, but is also limited in practice as it is complex in both computation and communication. However, in some situations complexity can be reduced by using equivalent iterative auctions like the English auction. We consider two properties that are needed for practical implementation of the VCG mechanism. One is called the buyers-are-substitutes property and relates to the existence of price equilibria, as shown by Bikhchandani and Ostroy [4]. The other is the concavity property which is nescessary for implementation of the primal-dual auction of De Vries et al. [53]. In section 5.3 we show both the buyers-are-substitutes property and concavity for the combinatorial auction that emerges from the capacitated assignment problem. Moreover, in section 5.4 we show that this problem is a special case of the models discussed by Bikhchandani and Ostroy [4] and De Vries et al. [53] and therefore their results can be applied to this setting. In section 5.5 we prove for two generalisations of the setting that the needed properties do not hold.

Chapter 6 deals with a parametric shortest path tree problem. The problem is a basic version of a problem class for designing incentive compatible and individual rational mechanisms. In section 1.4.2 we already showed how these kind of problems emerge from production planning. Section 6.2 defines the parametric problem as a shortest path problem where the lengths of the arcs are given by the products of arc dependent constants and node dependent variables. In section 6.3 we show an optimality condition. This condition has the nice property that it is in fact a local condition. The optimality condition suggests a local improvement algorithm which is presented in section 6.4. Unfortunately, this algorithm is not finite, which we show in an example. Section 6.5 presents an alternative formulation of the parametric shortest path tree problem where the parametric variables are eliminated. However, even here the exis- 
tence of a combinatorial algorithm that is running in polynomial time remains an open question.

Finally, in chapter 7 we present an overview of results in this thesis and conclusions. 



\section{Chapter 2}

\section{Auction Procurement over Multiple Periods: Combinatorial Auction}

\subsection{Introduction}

In this and the following two chapters we consider a company that needs to buy materials as input for its production process. Production takes place in a discrete number of periods, each having its own level of demand. This setting relates to the lot-sizing problem introduced in section 1.2.2. The company wishes to use an auction mechanism to buy this input as cheaply as possible. There may be different reasons to use a procurement auction, see Bichler et al. [3]. The first obvious reason is to gain from the competition between suppliers. If the company succeeds in inviting a lot of suppliers to compete, this may significantly reduce the price. Although important, it might not be easy to integrate competition and production planning:

"A (..) factor that may play an even more important role (..) is the effect of competition, although we acknowledge that it is the most difficult to integrate with models of short-term operational decisions such as production or procurement." (Yano and Gilbert [57])

However, increasing competition is not the only reason to use auctions. An auction that is tuned to the cost structure of suppliers, might as well benefit the suppliers. For example, Hohner et al. [24] used a combinatorial auction for the procurement process of the Mars corporation. This auction gave the suppliers the possibility of expressing their nonlinear costs for combinations of items. The auctions of Mars Inc. yielded consistent procurement cost savings, but: 
"We don't mean that suppliers are selling to us below cost. The efficiencies come from matching supplier capabilities and the company's needs and thus increasing suppliers' margins (..).” (Hohner et al. [24, page 32].)

So, the use of auctions may as well be advantageous for the suppliers by tuning capabilities and needs. This means that increasing the efficiency of the whole chain may benefit all links. The article of Hohner et al. [24] is one of very few where the auction is designed to achieve this tuning, as pointed out by Elmaghraby [10]:

"A serious omission from the procurement auctions literature is the important interaction between (..) suppliers' costs and the appropriate design of an auction."

On the contrary, most procurement auctions are just single-item auctions:

"Nearly all of the procurement auctions being run today in the private sector are single unit English auctions." (Bichler et al. [3])

Pinker et al. [41] conclude with (among others) the following area that is of interest in future research:

"Integrating auctions into the ongoing operations of the firm and quantifying the effects of auctions on critical business decisions such as procurement, marketing, production and inventory control, and supply chain management."

We may conclude from these quotes that there is a challenge in combining the procurement process and short-term production planning. In particular, we consider the use of procurement auctions in the multi-period production setting that is described by the lot-sizing model. There is a lot of literature on procurement auctions, see for a recent exposition Bichler et al. [3], including a description of the industrial procurement auction conducted at Mars, Inc., which was presented by Hohner et al. [24]. Furthermore, Tunca and $\mathrm{Wu}$ [51] provide a number of examples of companies and government organizations that make use of procurement auctions, including SUN Microsystems, Hewlett-Packard, IBM, Samsung, and Lucent. However, none of these papers focuses on the multi-period auction case. There are not many papers on this topic. In fact, in the procurement auction literature, the phrase "multi-period procurement" almost invariably refers to auctioning items sequentially via a series of single-period auctions, as in Elmaghraby [11], rather than auctioning them simultaneously via a single auction 
for multiple periods, as we will do here. One exception is by Kameshwaran et al. [26], who touch on the idea of a single auction for multiple periods in passing. They are mainly concerned with an auction for procuring heterogeneous items in a single period setting where the synergy across items is not strong. In their auction, rather than submit combinatorial bids, each supplier submits a single discount bid, which consists of the cost for each item of demand he offers to supply, together with a discount depending on the number of items actually supplied. The authors point out, however, that in a multi-period procurement scenario these discount bids would not be appropriate, as capacity constraints imply that the combination of items might be more important than the number of items. If demand in each period is considered as an indivisible item, then the problem reduces to procurement of multiple items, and in this case, a combinatorial bid can express the supplier's cost function more efficiently than a discount bid.

In this chapter we show that a combinatorial auction is an appropriate auction for multi-period production settings, i.e. it fits the cost structure implied by lot-sizing problems. This is shown in section 2.2. The combinatorial auction allows bidders to fully incorporate their cost structure, in particular balancing setup and holding costs. As a consequence, a combinatorial auction using the rules of the VCG mechanism will yield the cost minimizing production plan of the lot-sizing problem. Two extreme solutions of the lot-sizing problem can be related to more restricted auction settings. The first extreme solution is where all production takes place in the first period. In this solution only one setup is needed but holding costs are maximal. A corresponding auction is an auction where demand of all periods is auctioned and supplied at the beginning of the planning horizon. We will call this auction the stock auction. The other extreme solution is where all demand is produced just-in-time, which minimizes holding costs but needs a setup in every period. This solution corresponds to organizing an auction in each period, assuming that suppliers do not speculate on future demand. We refer to this setting as a series of separate auctions. First results from this and the next two chapters were presented in Romero Morales, Lok, and Steinberg [44, 45].

In section 2.3 we show that a combinatorial auction compromises the two alternative auction mechanisms. However, each alternative may be preferred over the combinatorial auction in specific settings. This raises the question whether we can make a measured choice between the three auction mechanisms. In chapters 3 and 4 we compare the combinatorial auction with separate auctions and one stock auction respectively. 


\subsection{Combinatorial structure}

In this chapter we consider the very common production setting where suppliers face lot-sizing problems, i.e. have production capacity in different periods. Unlike most procurement auctions, as pointed out in the previous section, we will allow the suppliers to incorporate all information about their costs (e.g., economies of scale in production and capacity restrictions) when submitting their respective bids. From the following examples it becomes clear that the cost structure implied by lot-sizing problems has a combinatorial character with respect to the demand vector.

Recall the lot-sizing problem (LSP) from section 1.2.2. We defined $T$ as the planning horizon. For each period $t$ we had $R_{t}$ the production capacity, $p_{t}$ the unit production cost, $h_{t}$ the unit inventory holding cost, and $f_{t}$ the setup cost if production takes place. Let $Q$ denote a supply vector, i.e. $Q_{t}$ is the amount supplied in period $t \in\{1, \ldots, T\}$. Now, let $C(Q)$ be the minimum costs for supplying vector $Q$, i.e. the optimal value of (LSP) when the vector of demand is equal to $Q$. The cost function is said to have a nonlinear or combinatorial character if $C\left(Q^{\prime}+Q^{\prime \prime}\right) \neq C\left(Q^{\prime}\right)+C\left(Q^{\prime \prime}\right)$. If $C\left(Q^{\prime}+Q^{\prime \prime}\right)<$ $C\left(Q^{\prime}\right)+C\left(Q^{\prime \prime}\right)$ we say that $Q^{\prime}$ and $Q^{\prime \prime}$ are complements, i.e. when produced together they gain economies of scale. We can illustrate this with a simple example.

Example 2.2.1 Suppose we have a lot-sizing problem with two periods, i.e. $T=2$. In Table 2.1 the parameter values are given. Note that the inventory holding costs are very

\begin{tabular}{c|rr} 
& $t=1$ & $t=2$ \\
\hline$p_{t}$ & 1 & 1 \\
$f_{t}$ & 5 & 5 \\
$h_{t}$ & 9 & - \\
$R_{t}$ & 4 & 4 \\
\hline
\end{tabular}

Table 2.1: Data for Example 2.2.1

expensive compared to the unit production costs and the setup costs. In fact, it is not profitable to hold any inventory, unless enforced by capacity restrictions. Consider the supply vector $Q=(2,2)$. The cheapest way to supply this demand, is to produce each unit just-in-time, so $C(Q)=14$. For $Q^{\prime}=Q^{\prime \prime}=(1,1)$ we have that $C\left(Q^{\prime}\right)=12$. Note that $Q=Q^{\prime}+Q^{\prime \prime}$. Then,

$$
C\left(Q^{\prime}+Q^{\prime \prime}\right)<C\left(Q^{\prime}\right)+C\left(Q^{\prime \prime}\right)
$$

as in the right hand side we have paid twice the fixed costs. 
So the setup costs in the lot-sizing problem may give the costs a complementary character. If $C\left(Q^{\prime}+Q^{\prime \prime}\right)>C\left(Q^{\prime}\right)+C\left(Q^{\prime \prime}\right)$ we say that $Q^{\prime}$ and $Q^{\prime \prime}$ are substitutes, i.e. both compete for the same capacity. We have a similar example as above.

Example 2.2.2 Suppose we have a lot-sizing problem with two periods, i.e. $T=2$. In Table 2.2 the parameter values are given. Again, consider a supply vector $Q=(2,2)$. For

\begin{tabular}{r|rr} 
& $t=1$ & $t=2$ \\
\hline$p_{t}$ & 1 & 1 \\
$f_{t}$ & 0 & 0 \\
$h_{t}$ & 2 & - \\
$R_{t}$ & 4 & 1 \\
\hline
\end{tabular}

Table 2.2: Data for Example 2.2.2

a unit of demand in period 2 the cheapest solution is to produce in period 2 as otherwise inventory holding costs have to be paid. However, there is only capacity for producing one unit in period 2. The cheapest way to supply $Q_{2}=2$, is to produce one unit in each period, so $C(Q)=6$. For $Q^{\prime}=Q^{\prime \prime}=(1,1)$ we have that $C\left(Q^{\prime}\right)=C\left(Q^{\prime \prime}\right)=2$. Note again that $Q=Q^{\prime}+Q^{\prime \prime}$. Then,

$$
C\left(Q^{\prime}+Q^{\prime \prime}\right)>C\left(Q^{\prime}\right)+C\left(Q^{\prime \prime}\right)
$$

as in the right hand side we considered $Q^{\prime}$ and $Q^{\prime \prime}$ separately, both using the scarce capacity in period 2.

So, even though we are discussing the procurement of only one type of item, the costs of the suppliers have a combinatorial character due to setup costs and capacities.

\subsection{Compromising setup and holding costs}

\subsubsection{Combinatorial VCG auction}

In our procurement auction we have one buyer and a set of suppliers $\mathcal{S}$. Each supplier can supply the demand of one or more periods from the planning horizon of $T$ periods. In period $t$ the buyer needs $D_{t}$ units of input, where $t \in\{1, \ldots, T\}$ and $D=\left(D_{t}\right) \in \mathbb{R}^{T}$ denotes the vector of demands. It is assumed that each $D_{t}$ has to be produced at once by a single supplier, at any time not later than $t$. The suppliers individually submit bids in the form of a bid price together with a $T$-vector representing an offer to supply specific 
quantities of units in periods $1, \ldots, T$, where the quantity in period $t$ is not restricted to being zero or $D_{t}$, but can be the sum of any subset of the buyer's demands $D_{\tau}$ for $\tau \in\{t, t+1, \ldots, T\}$. However, supplier $s \in \mathcal{S}$ is restricted by its production capacity $R_{s t}$ in period $t$. We have the assumption that, at every point in the planning horizon, there exists sufficient production capacity from the suppliers to meet the total demand requirements to date, i.e., $\sum_{s \in \mathcal{S}} \sum_{t=1}^{\tau} R_{s t} \geq \sum_{t=1}^{\tau} D_{t}$, for all $\tau \in\{1, \ldots, T\}$. In other words, satisfying the demand requirements from the suppliers is a feasible problem for the buyer.

Supplier $s$ faces a setup cost $f_{s t}$ if he produces in period $t$, a unit production cost $p_{s t}$ in period $t$, and a unit inventory holding cost $h_{s}$ per period. The only cost faced by the buyer is a unit inventory holding cost of $H$ per period. The standard supply chain assumption that $H \geq h_{s}$ for all $s \in \mathcal{S}$ applies. This assumption implies that if the suppliers submit all possible bids truthfully, then there is always an optimal allocation such that the suppliers hold all inventory. Therefore, we can assume without loss of generality that only suppliers keep inventory when we use the combinatorial auction.

In the following we recall the VCG mechanism as described in section 1.3.2.2, specified in the setting and notation of the procurement auction, i.e. where the bidders are suppliers that face costs when producing. The VCG mechanism is a one shot auction where the buyer announces its demand vector and all suppliers submit at once all bids on any subset of the demand vector. For example, if the buyer needs $D=(1,1)$, all suppliers will submit their prices for the vectors $(1,1),(1,0)$ and $(0,1)$, where the bidprices may be infinite if capacity is insufficient.

Due to the incentive characteristics of the VCG mechanism, we may assume that all suppliers bid their true costs for every bid. After receiving all bids the buyer computes a cost-minimizing allocation of sets of goods to the suppliers. Because of the combinatorial character of the costs, the buyer may select at most one winning bid from each supplier. Each supplier $s \in \mathcal{S}$ receives a payment equal to its costs $C_{\mathcal{S}}^{s}$ plus a surplus. Specifically, the surplus for supplier $s$ is the marginal contribution or marginal product of the supplier to the total costs. This is the difference between the total costs had supplier $s$ not participated in the auction, $C_{\mathcal{S} \backslash\{s\}}$, and the actual costs $C_{\mathcal{S}}$. In total, the buyer pays

$$
\sum_{s \in \mathcal{S}}\left[C_{\mathcal{S}}^{s}+\left(C_{\mathcal{S} \backslash\{s\}}-C_{\mathcal{S}}\right)\right]=C_{\mathcal{S}}+\sum_{s \in \mathcal{S}}\left(C_{\mathcal{S} \backslash\{s\}}-C_{\mathcal{S}}\right),
$$

where, by definition, $C_{\mathcal{S}}=\sum_{s \in \mathcal{S}} C_{\mathcal{S}}^{s}$, the total costs of the suppliers. Observe that the marginal product of any supplier that does not win any demand equals zero. Then if $\mathcal{W}$ denotes the set of winning suppliers, the total payment of our auction can equivalently 
be written as

$$
C_{\mathcal{S}}+\sum_{s \in \mathcal{W}}\left(C_{\mathcal{S} \backslash\{s\}}-C_{\mathcal{S}}\right)
$$

As a consequence the payment reduces to $C_{\mathcal{S} \backslash\left\{s_{1}\right\}}$ if there is only one winner $s_{1}$, i.e., $\mathcal{W}=\left\{s_{1}\right\}$.

\subsubsection{Stock Auction and Separate Auctions}

As said, the incorporation of the suppliers' costs structures may be advantageous for both the suppliers and the buyer. The question is, what is the advantage from using the combinatorial auction. Of course, this depends on the mechanism that is replaced by the combinatorial auction.

The first alternative that we consider is the stock auction. In the stock auction total demand is auctioned at the beginning of the planning horizon, ignoring production ability in later periods. As a consequence this auction maximizes the inventory levels. The stock auction allows suppliers to achieve economies of scale, but gives no flexibility in production timing. Our second alternative, is to run a series of separate auctions for each period separately. These separate auctions have minimal (zero) inventory levels, but a maximal number of setups as production takes place in each period. The two alternative auctions also correspond to the extreme solutions of the lot-sizing problem: producing everything at once, minimizing the number of setups, versus producing everything just-in-time, minimizing inventory levels. So, like the lot-sizing problem itself, a main part of the combinatorial auction is to find a balance between holding costs and setup costs. In the following we will investigate the stock auction and the separate auctions, where we assume the VCG mechanism in both cases.

The stock auction runs in a similar way as the combinatorial auction. However, production is only allowed in the first period. This means that the buyer faces inventory holding costs itself for any demand $D_{t}$, with $t>1$. The VCG payment in the stock auction is equal to

$$
C_{\mathcal{S}}^{I}+\sum_{s \in \mathcal{S}}\left(C_{\mathcal{S} \backslash\{s\}}^{I}-C_{\mathcal{S}}^{I}\right)
$$

where $C_{\mathcal{S}}^{I}$ is the lowest cost at which the set of suppliers $\mathcal{S}$ can supply $D$, when production is only allowed in period 1 . (Note that we distinguish this cost function of the stock auction by a superscript $I$.) In addition to these costs, the buyer has to pay for holding inventory, i.e. $\sum_{t=2}^{T}(t-1) H D_{t}$. Note that now the buyer bears the holding costs directly, where we could assume that the suppliers kept inventory in the combinatorial auction. Since any production planning for the stock auction is also feasible for the 
combinatorial auction, and since the buyer's holding costs are at least as large as the suppliers' holding costs we know that

$$
C_{\mathcal{S}}^{I}+\sum_{t=2}^{T}(t-1) H D_{t} \geq C_{\mathcal{S}} .
$$

In the series of separate auctions, the VCG mechanism boils down to the Vickrey auction, as we assumed for all three types of auctions that $D_{t}$ is indivisible. Therefore, the price to be paid equals the second best bid. Note that $f_{s t}+p_{s t} D_{t}$ is the total production costs that supplier $s$ faces if he wins $D_{t}$. Obviously, these costs are only defined for the suppliers having enough capacity in period $t$ to fulfill $D_{t}$. Therefore, let $\mathcal{F}_{t}$ be the set suppliers who can produce demand $D_{t}$ in period $t$. Then

$$
\left(f_{\cdot t}+p_{\cdot t} D_{t}\right)^{(n)}
$$

denotes the $n$-th lowest value in $\left\{f_{s t}+p_{s t} D_{t}\right\}_{s \in \mathcal{F}_{t}}$. The total payment in the $T$ different separate auctions is equal to

$$
\sum_{t=1}^{T}\left(f_{\cdot t}+p_{\cdot t} D_{t}\right)^{(2)} .
$$

Let $\pi^{C}, \pi^{I}$ and $\pi^{S}$ be the total procurement costs using respectively the combinatorial auction, the stock auction, and the series of separate auctions. These costs include the inventory holding costs, where we can assume without loss of generality that the suppliers keep inventory in the combinatorial auction, the buyer keeps inventory in the stock auction, and no inventory is kept in the series of separate auctions. For the combinatorial auction and the series of separate auctions the procurement costs are just the total auction payments given above. In the stock auction the buyer receives all supplies in the first period and has to hold in inventory the items that are needed in later periods. The total procurement costs are therefore

$$
\pi^{I}=C_{\mathcal{S}}^{I}+\sum_{s \in \mathcal{S}}\left(C_{\mathcal{S} \backslash\{s\}}^{I}-C_{\mathcal{S}}^{I}\right)+\sum_{t=2}^{T}(t-1) H D_{t} .
$$

\subsubsection{Comparison of the auction alternatives}

In this section we show by examples that either of the three auction mechanisms may be the cheapest for the buyer. We start with an example in which the combinatorial auction is indeed the best option for the buyer. 
Example 2.3.1 (Combinatorial auction is cheapest solution) Consider a scenario where the buyer requires input over two time periods, with demand $D_{t}=1$ in each period $t$ and the buyer's holding cost is $H=2$. There are three suppliers, $s=1,2,3$, each with holding $\operatorname{cost} h_{s}=1$ and setup cost $f_{s t}=3$. The rest of the suppliers' data are shown in Table 2.3. In the combinatorial auction setting we have three options for producing $D$ :

\begin{tabular}{r|rrrr}
$s$ & $p_{s 1}$ & $R_{s 1}$ & $p_{s 2}$ & $R_{s 2}$ \\
\hline 1 & 5 & 1 & 5 & 1 \\
2 & 10 & 2 & 10 & 1 \\
3 & 15 & 2 & 15 & 1 \\
\hline
\end{tabular}

Table 2.3: Data for Example 2.3.1

- Produce everything in period 1, using the cheapest supplier that can produce both units of demand, i.e. using economies of scale. This has a cost of $3+2 \times 10+1=24$.

- Produce everything in period 1, using the cheapest supplier and the second cheapest supplier as the former has a limited capacity. The costs are $3+5+3+10+1=22$.

- Produce $D_{1}$ in period 1 , and produce $D_{2}$ in period 2 , having cost $3+5+3+5=16$.

So $C_{\mathcal{S}}=16$, where supplier 1 produces both units of demand, just-in-time. Recall from section 2.3.1 that the one-winner case has a payment equal to $C_{\mathcal{S} \backslash\{1\}}$. The payment in this combinatorial auction is therefore $C_{\mathcal{S} \backslash\{1\}}=3+2 \times 10+1=24$.

For having two separate auctions, the total production costs are again $3+5+3+5=16$, and the total procurement costs are equal to $3+10+3+10=26$.

In the stock auction setting we have two options for producing $D$ :

- Produce everything in period 1 , using economies of scale, with cost $3+2 \times 10=23$.

- Produce everything in period 1 , without economies of scale, with cost $3+5+3+10=$ 21.

So $C_{\mathcal{S}}^{I}=21$, and we have two winning suppliers, 1 and 2. Clearly, $C_{\mathcal{S} \backslash\{1\}}^{I}=3+2 \times 10=23$ and $C_{\mathcal{S} \backslash\{2\}}^{I}=3+5+3+15=26$. The total payment in the stock auction is $21+(23-$ $21)+(26-21)=28$, giving total procurement cost of $28+2=30$, including holding costs borne by the buyers. 
In the next example we show that in some cases the series of separate auctions are cheaper than the combinatorial auction.

Example 2.3.2 (Separate auctions are cheapest solution) Consider a scenario where the buyer requires an item over two time periods, with demand $D_{t}=1$ in each period $t$ and the buyer's holding cost is $H=2$. There are five suppliers, $s=1, \ldots, 5$, each with holding cost $h_{s}=1$. Each supplier has production capacity 1 in one of the periods and no capacity in the other period. None of the suppliers faces setup costs in either period. The supplier capacities and unit production costs are shown in Table 2.4. In the series of

\begin{tabular}{r|rrr}
$s$ & $R_{s 1}$ & $R_{s 2}$ & $p_{s t}$ \\
\hline 1 & 1 & 0 & 5 \\
2 & 1 & 0 & 9 \\
3 & 1 & 0 & 13 \\
4 & 0 & 1 & 14 \\
5 & 0 & 1 & 15 \\
\hline
\end{tabular}

Table 2.4: Data for Example 2.3.2

separate auctions setting, there are two auctions. In the first period auction, supplier 1 is the (single) winner having production cost 5 and receiving the second best bid which is 9 . Similarly, in the second period, supplier 4 is the cheapest supplier and receives the second best bid, i.e. 15. (Note that we assume that no inventory is possible in this setting.) We conclude that the total procurement costs of the buyer are $9+15=24$.

In the combinatorial auction setting the suppliers have the possibility to hold inventory. This means that although suppliers 1, 2 and 3 have no capacity in period 2, they can in fact choose to produce in period 1 and hold the item in inventory until period 2, at an effective period 2 production cost of 6,10 and 14 , respectively. The winning bids are $(1,0)$ from supplier 1 with cost 5 , together with $(0,1)$ from supplier 2 with cost 10 , so $C_{\mathcal{S}}=15$. Furthermore, it is easy to see that $C_{\mathcal{S} \backslash\{1\}}=9+14=23$ and $C_{\mathcal{S} \backslash\{2\}}=5+14=19$. From this we get a total payment of $15+(23-15)+(19-15)=27$, which is more than the total buyer payment in the series of separate auctions. We may observe that the competition for the second period demand increases and therefore we obtain a better price, however this has a negative effect on the payments related with the first period demand.

In the stock auction, the winning bids are $(1,0)$ from supplier 1 with cost 5 , together with $(1,0)$ from supplier 2 with cost 9 , so $C_{\mathcal{S}}=14$. Furthermore, it is not hard to see that $C_{\mathcal{S} \backslash\{1\}}=9+13=22$ and $C_{\mathcal{S} \backslash\{2\}}=5+13=18$. From this we get a total payment of $14+(22-14)+(18-14)=26$, so the total costs of the buyer including holding costs 
are $26+2=28$. So, in this example the procurement costs are the lowest in the series of separate auctions (24), and the highest in the stock auction (28). Note that in case the buyer had the same holding costs as the suppliers, that the total procurement costs of the combinatorial auction and the stock auction would have been the same.

In the next example we show that there exist instances in which the stock auction is cheaper than the combinatorial auction.

Example 2.3.3 (Stock auction is cheapest solution) Consider a scenario where the buyer requires an item over two time periods, with demand $D_{t}=1$ in each period $t$, where the buyer's holding cost is $H=1$. There are four suppliers, $s=1, \ldots, 4$. All suppliers have setup costs equal to 2, and inventory holding costs equal to 1 . The rest of the suppliers' data are shown in Table 2.5. First have a look at the case of the stock auction. The optimal

\begin{tabular}{r|rrrr}
$s$ & $p_{s 1}$ & $R_{s 1}$ & $p_{s 2}$ & $R_{s 2}$ \\
\hline 1 & 9 & 1 & 13 & 1 \\
2 & 10 & 2 & 12 & 1 \\
3 & 10 & 2 & 11 & 1 \\
4 & 12 & 2 & 8 & 1 \\
\hline
\end{tabular}

Table 2.5: Data for Example 2.3.3

allocation of the stock auction will use economies of scale or not, i.e.,

$$
C_{\mathcal{S}}^{I}=\min \{2+2 \times 10,2+9+2+10\}=\min \{22,23\}=22 .
$$

This shows that the best option is to use economies of scale of supplier 2. The payment of the stock auction is equal to the costs made if supplier 2 is excluded. As supplier 3 has the same costs and the same capacity in period 1, the payment is also 22. Together with holding costs, the buyer has a total procurement cost of 23.

Now consider the combinatorial auction. This auction may improve the stock auction by letting $D_{2}$ be produced in period 2. Therefore,

$$
C_{\mathcal{S}}=\min \{22+1,2+9+2+8\}=\min \{23,21\}=21,
$$

where supplier 1 is the winner of $D_{1}$ and supplier 4 is the winner of $D_{2}$. After we eliminate supplier 1, we could again produce each unit in each corresponding period, with a one unit increase with respect to the optimal allocation, i.e., 22 or produce both units in period 1 and then we will face the costs of the stock auction allocation, i.e., $22+1$, which is 
more expensive. However, when we eliminate supplier 4, there is no competitive supplier that can produce $D_{2}$ in period 2 and therefore, the best option is to produce both units in period 1 at a cost of 23. Summarizing the payment of the combinatorial auction is equal to $21+(22-21)+(23-21)=24$.

Finally, in two separate auctions the total production costs are $2+9+2+8=21$, while the buyer has to pay $2+10+2+11=25$. So, in this example the stock auction is the cheapest option, while the series of separate auctions is the most expensive one.

\subsubsection{Combinatorial auction compromises the alternatives}

In none of the previous examples the combinatorial auction is strictly more expensive than both the other two options. This is not a coincidence. To see this we combine the following two propositions.

Proposition 2.3.4 If the production plans of the combinatorial auction and the stock auction are equal, then we have $\pi^{C} \leq \pi^{I}$.

\section{Proof:}

By assumption the production plans and (as a consequence) the total inventory levels are equal in both auctions, where the buyer holds inventory in the stock auction and the suppliers hold inventory in the combinatorial auction. Therefore, the only difference in total costs relates to the unit holding costs. In the stock auction inventory is hold at the most expensive level of the buyer, therefore

$$
C_{\mathcal{S}}^{I}+\sum_{t=2}^{T}(t-1) H D_{t} \geq C_{\mathcal{S}} .
$$

Furthermore, let $\hat{H}\left(C_{\mathcal{S}}\right)$ denote the total inventory holding cost that is part of $C_{\mathcal{S}}$. Then, as there is only a difference in inventory costs, we have

$$
C_{\mathcal{S}}^{I}+\hat{H}\left(C_{\mathcal{S}}\right)=C_{\mathcal{S}}
$$

Using the previous expressions we can write:

$$
\begin{aligned}
\pi^{I}-\pi^{C} & =C_{\mathcal{S}}^{I}+\sum_{s \in \mathcal{S}}\left(C_{\mathcal{S} \backslash\{s\}}^{I}-C_{\mathcal{S}}^{I}\right)+\sum_{t=2}^{T}(t-1) H D_{t}-\left[C_{\mathcal{S}}+\sum_{s \in \mathcal{S}}\left(C_{\mathcal{S} \backslash\{s\}}-C_{\mathcal{S}}\right)\right] \\
& \geq \sum_{s \in \mathcal{S}}\left(C_{\mathcal{S} \backslash\{s\}}^{I}-C_{\mathcal{S}}^{I}\right)-\sum_{s \in \mathcal{S}}\left(C_{\mathcal{S} \backslash\{s\}}-C_{\mathcal{S}}\right) \\
& =\sum_{s \in \mathcal{S}}\left[\left(C_{\mathcal{S} \backslash\{s\}}^{I}+\hat{H}\left(C_{\mathcal{S}}\right)-C_{\mathcal{S} \backslash\{s\}}\right)+\left(C_{\mathcal{S}}-C_{\mathcal{S}}^{I}-\hat{H}\left(C_{\mathcal{S}}\right)\right)\right]
\end{aligned}
$$




$$
\begin{aligned}
& =\sum_{s \in \mathcal{S}}\left(C_{\mathcal{S} \backslash\{s\}}^{I}+\hat{H}\left(C_{\mathcal{S}}\right)-C_{\mathcal{S} \backslash\{s\}}\right) \\
& \geq 0
\end{aligned}
$$

The first inequality follows from (2.7). The next equality follows from rearranging terms after adding $\hat{H}\left(C_{\mathcal{S}}\right)-\hat{H}\left(C_{\mathcal{S}}\right)$, and the last equality follows from (2.8). Finally, the last inequality holds because any solution for the stock auction is also feasible in the combinatorial auction. Thus, the desired inequality holds.

Proposition 2.3.5 If the production plans of the combinatorial auction and the series of separate auctions are equal, then we have $\pi^{C} \leq \pi^{S}$.

\section{Proof:}

Let $s \in \mathcal{S}$ be a winner in the combinatorial auction and let $T_{s}$ be the set of periods in which $s$ is the winning supplier. By assumption we have $C_{\mathcal{S}}=\sum_{t=1}^{T}\left(f_{\cdot t}+p_{\cdot t} D_{t}\right)^{(1)}$. If winner $s$ is excluded, it is a feasible solution to produce its winning demands by the second best supplier in the same period, so we have

$$
C_{\mathcal{S} \backslash\{s\}}-C_{\mathcal{S}} \leq \sum_{t \in T_{s}}\left[\left(f_{\cdot t}+p_{\cdot t} D_{t}\right)^{(2)}-\left(f_{\cdot t}+p_{\cdot t} D_{t}\right)^{(1)}\right]
$$

and thus

$$
\sum_{s \in \mathcal{S}}\left(C_{\mathcal{S} \backslash\{s\}}-C_{\mathcal{S}}\right) \leq \sum_{t=1}^{T}\left[\left(f_{\cdot t}+p_{\cdot t} D_{t}\right)^{(2)}-\left(f_{\cdot t}+p_{\cdot t} D_{t}\right)^{(1)}\right] .
$$

With this we can write:

$$
\begin{aligned}
\pi^{S}-\pi^{C}= & \sum_{t=1}^{T}\left(f_{\cdot t}+p_{\cdot t} D_{t}\right)^{(2)}-\left[C_{\mathcal{S}}+\sum_{s \in \mathcal{S}}\left(C_{\mathcal{S} \backslash\{s\}}-C_{\mathcal{S}}\right)\right] \\
= & \sum_{t=1}^{T}\left(f_{\cdot t}+p_{\cdot t} D_{t}\right)^{(2)}-\left[\sum_{t=1}^{T}\left(f_{\cdot t}+p_{\cdot t} D_{t}\right)^{(1)}+\sum_{s \in \mathcal{S}}\left(C_{\mathcal{S} \backslash\{s\}}-C_{\mathcal{S}}\right)\right] \\
\geq & \sum_{t=1}^{T}\left(f_{\cdot t}+p_{\cdot t} D_{t}\right)^{(2)} \\
& \quad-\left[\sum_{t=1}^{T}\left(f_{\cdot t}+p_{\cdot t} D_{t}\right)^{(1)}+\sum_{t=1}^{T}\left[\left(f_{\cdot t}+p_{\cdot t} D_{t}\right)^{(2)}-\left(f_{\cdot t}+p_{\cdot t} D_{t}\right)^{(1)}\right]\right] \\
= & 0,
\end{aligned}
$$

and the desired inequality holds. 
These two propositions imply that if $T=2$ the combinatorial auction is never strictly worse than both the stock auction and the series of separate auctions.

Corollary 2.3.6 If $T=2$, then $\pi^{C}$ is never strictly larger than both $\pi^{I}$ and $\pi^{S}$.

Proof:

Consider the combinatorial auction. In any case $D_{1}$ is produced in period 1. For $D_{2}$ there are two options, it is either produced in period 1 or in period 2. This implies that we have either the case of Proposition 2.3.4 or of Proposition 2.3.5.

In the two period setting, the combinatorial auction is either the best solution (see Example 2.3.1) or a compromise between the stock auction and a series of separate auctions (Corollary 2.3.6). So, indeed we may regard the combinatorial auction as a good compromise. In the next two chapters we compare the procurement costs of the combinatorial auction and its two alternatives.

In chapter 3 we compare the combinatorial auction with the series of separate auctions. It appears that in the uncapacitated setting, the combinatorial auction is always the cheapest auction format for the buyer. For capacitated problems it may happen that the series of separate auctions is cheaper than a combinatorial auction. However, for a two-period scenario we can still show that for many instances the combinatorial auction is still the cheapest. Moreover, we show that in expectation the combinatorial auction is at most as expensive as the series of separate auctions.

In chapter 4 we compare the combinatorial auction with the stock auction. Again, it appears that in the uncapacitated setting, the combinatorial auction is always the cheapest auction format for the buyer. For a two-period capacitated scenario, the stock auction may be the cheapest solution, but we are able to bound the loss of using the combinatorial auction instead. First this loss is bounded by one setup cost, and second, it is bounded by the level of competition in the second period. This means that if the costs of the different suppliers are close to each other, the possible loss is also small. 


\section{Chapter 3}

\section{Combinatorial Auction versus Separate Auctions}

\subsection{Introduction}

In this chapter we compare the combinatorial auction and the series of separate auctions. The combinatorial auction will increase production efficiency as compared to the series of separate auctions, as it will allow the suppliers to incorporate more information about their costs. In other words, suppliers can produce more cheaply and therefore bid more competitively against each other. Moreover, a combinatorial auction will allow the buyer to purchase units in advance of when required. This can be advantageous if supplier production capacity is scarce in some periods, or if production costs vary from period to period.

Thus, intuitively one might expect the combinatorial auction to always be advantageous for the buyer, as the buyer will receive better bids, i.e., lower prices, which can be more flexibly combined with each other. This would allow the buyer to satisfy his demand schedule at lower cost than with the series of separate auctions.

From Example 2.3.2 we know that, to the contrary, there are cases in which the buyer will be worse off using a combinatorial auction. So, in some cases, in spite of the increase in production efficiency the buyer needs in fact to pay more. This somewhat counter-intuitive result is reminiscent of the well-known result of Hart [22], who considers the consequences in a market structure of permitting trades that were previously prohibited, where "our intuition tells us that the introduction of additional markets ought to make people better off," but provides an example in which this is not the case. 
The counterintuitive result can arise from two factors. The first is supplier transformation, i.e. holding inventory. Although the aim of the supplier may be only to lower its own production costs, supplier production in advance of demand will have the added effect of transferring supplier competition to other periods. By using capacity for future periods the capacity is out of competition for the period it is available, while increasing competition in future periods. The net effect can both be positive or negative. The second factor is that in general a supplier can have production capacity in multiple periods. Although this overlap in suppliers is of no importance for the separate auctions, it can be detrimental to the buyer in the combinatorial auction. The joint availability of production capacity over multiple periods may place the supplier in a more powerful position with respect to the buyer. In summary we have the following effects:

- Transformation effect, which has two components:

- production efficiency effect (always positive for the buyer), and

- transferred competition effect (can be positive or negative for the buyer).

- Joint capacity effect (always negative for the buyer).

In section 3.2 we show that in the uncapacitated case the combinatorial auction is never more expensive than having a series of separate auctions. In section 3.3 we delimit for a two-period setting some cases with the same property for the capacitated scenario. Moreover, we show that the expected savings from using a combinatorial auction instead of a series of separate auctions are nonnegative.

\subsection{Uncapacitated case}

In this section we assume that the capacities of the suppliers are not binding, i.e. $R_{s t} \geq$ $\sum_{\tau=t}^{T} D_{\tau}$, for all $t \in\{1, \ldots, T\}$ and $s \in \mathcal{S}$. Under this scenario, we can show that the combinatorial auction results in a cost to the buyer that is less than or equal to that which would result under the $T$ separate auctions. Recall that each $D_{t}$ has to be produced at once by a single supplier.

Theorem 3.2.1 If the capacities are not binding, we have $\pi^{C} \leq \pi^{S}$.

\section{Proof:}

Consider the optimal allocation for the combinatorial auction, and recall that $C_{\mathcal{S}}^{s}$ denotes the costs incurred by supplier $s \in \mathcal{S}$, with $C_{\mathcal{S}}=\sum_{s \in \mathcal{S}} C_{\mathcal{S}}^{s}$. Let $T_{s} \subseteq\{1,2, \ldots, T\}$ be 
the index set of the demands won by supplier $s$. Recall that $\left(f_{\cdot t}+p_{\cdot t} D_{t}\right)^{(n)}$ is the $n$-th lowest in $\left\{f_{s t}+p_{s t} D_{t}\right\}_{s \in \mathcal{S}}$. Define $\left(f_{\cdot t}+p_{\cdot t} D_{t}\right)^{-s,(1)}$ as the cheapest value in $\left\{f_{r t}+p_{r t} D_{t}\right\}_{r \in \mathcal{S} \backslash\{s\}}$, i.e. if $s$ is excluded. Then, we have that

$$
C_{\mathcal{S} \backslash\{s\}}-C_{\mathcal{S}} \leq \sum_{t \in T_{s}}\left(f_{\cdot t}+p_{\cdot t} D_{t}\right)^{-s,(1)}-C_{\mathcal{S}}^{s}
$$

as if $s$ is excluded, it is feasible to produce $D_{t}, t \in T_{s}$, by the cheapest player according to $\left\{f_{r t}+p_{r t} D_{t}\right\}_{r \in \mathcal{S} \backslash\{s\}}$. (Notice that this is a quick way to find the costs of a feasible but probably non-optimal solution. Moreover, we may overestimate the costs of this feasible solution if we already paid for some of those setups for the demands won by the other suppliers. Nonetheless, this bound suffices to prove the result.)

Using this inequality for all $s \in \mathcal{S}$, we have that

$$
\begin{aligned}
C_{\mathcal{S}}+\sum_{s \in \mathcal{S}}\left(C_{\mathcal{S} \backslash\{s\}}-C_{\mathcal{S}}\right) & \leq C_{\mathcal{S}}+\sum_{s \in \mathcal{S}}\left(\sum_{t \in T_{s}}\left(f_{\cdot t}+p_{\cdot t} D_{t}\right)^{-s,(1)}-C_{\mathcal{S}}^{s}\right) \\
& =\sum_{s \in \mathcal{S}} \sum_{t \in T_{s}}\left(f_{\cdot t}+p_{\cdot t} D_{t}\right)^{-s,(1)} \\
& \leq \sum_{s \in \mathcal{S}} \sum_{t \in T_{s}}\left(f_{\cdot t}+p_{\cdot t} D_{t}\right)^{(2)} \\
& =\sum_{t=1}^{T}\left(f_{\cdot t}+p_{\cdot t} D_{t}\right)^{(2)},
\end{aligned}
$$

which proves the desired result.

We may relate this result to the effects of transformation and joint capacities. Note that in the uncapacitated case we do not have an adverse effect from transferred competition: One can use production capacity in period $s$ for period $t(s<t)$ without limiting production possibilities. Therefore, Theorem 3.2.1 says that the (adverse) joint capacity effect is smaller than or equal to the transformation effect. However, in Example 3.2.2 we show that in the uncapacitated case the joint capacity effect can be quite large. In fact, the suppliers are able to claim the whole cost reduction from using the combinatorial auction instead of the series of separate auctions. This leaves the buyer with no benefit at all.

Example 3.2.2 (Joint capacity effect) Consider the scenario where the buyer requires an item over two time periods, with demand $D_{t}=1$ in each period $t$. There are four suppliers, i.e. $\mathcal{S}=\{1,2,3,4\}$. The suppliers have unrestricted capacity and no setup costs. 


\begin{tabular}{r|rr}
$s$ & $p_{s 1}$ & $p_{s 2}$ \\
\hline 1 & 5 & 9 \\
2 & 7 & 9 \\
3 & 9 & 7 \\
4 & 9 & 8 \\
\hline
\end{tabular}

Table 3.1: Data for Example 3.2.2

Furthermore, all suppliers have unit holding costs equal to 1, while the buyer has holding cost $H=2$. The suppliers' data are shown in Table 3.1.

First have a look at the case of two separate auctions. Total production costs are $5+7=$ 12 , while the procurement costs are $7+8=15$, i.e. the second best costs of the two periods. Introducing a combinatorial auction instead of the separate ones, induces a saving on the production costs as we may produce $D_{2}$ at a cost of $5+1$ instead of 7 . We can see that this saving in production costs is given to the buyer: As supplier 1 is the only winner, its payment is 14, the total production costs without this supplier. So, the buyer pays one unit less by using a combinatorial auction instead of a series of separate auctions.

However, due to the combined competition effect, it is not obvious that the buyer profits from this cost reduction. Suppose that suppliers 1 and 3 merge to one player with costs $(5,7)$. Then, the production costs of the combinatorial auction would remain 11 , but the payment would increase to $7+8=15$, the same as for the separate auctions. This shows that in some cases the suppliers are able to claim the whole cost reduction, leaving the buyer with no benefit at all.

In Example 2.3.2 we have shown that Theorem 3.2.1 does not hold, in general, in the capacitated case. In that example none of the suppliers has capacity over multiple periods, so there is no joint capacity effect. Consequently, the transformation effect has a net negative influence on the buyer's procurement costs.

\subsection{Capacitated case}

So far we have seen that the buyer always prefers the combinatorial auction to the series of separate ones in the uncapacitated case. However, Example 2.3.2 showed that separate auctions might be cheaper in the capacitated setting, even in the absence of setup costs. In this section we will have a closer look at the capacitated case. In Section 3.3.1 we consider a two-period problem with the same setup costs and the same inven- 
tory holding costs across all the suppliers. First, we delimit a set of problem instances for which the combinatorial auction is never more expensive than the separate auctions. Second, we show for uniform, identical and independent probability distributions of the production costs that in expectation the combinatorial auction is at most as expensive as a series of separate auctions.

The setting we consider is related to the work of Elmaghraby [11]. She considers two sequential procurement auctions for procurement of two (identical) items. The auctions have two types of bidders: global bidders that have capacity to produce both items, and small bidders that only have capacity for producing one item. It is assumed that all producers face the same fixed setup cost, but differ in their variable production cost and capacity. So global bidders may have economies of scale. In this setting Elmaghraby shows that the commonly held belief that increasing the number of bidders makes an auction more competitive' does not always hold: Adding small bidders to an auction with only global bidders may in some cases increase expected procurement costs. An increase in costs typically occurs when the small bidders have relatively high production costs, and hence do not win the auctions, but their presence results in the global bidders inflating their bids. Compared to our setting, Elmaghraby [11] applies a series of auctions to procure a single-period demand, where we introduce a multiperiod setting and the possibility of inventories. Furthermore, Elmaghraby examines the influence of the bidder population, where we focus on the auction format that can be applied.

Like Elmaghraby [11], in the following we will assume that all bidders have the same setup cost. Moreover, we will also assume that all bidders face the same inventory holding costs. Slagmulder et al. [47, 48] described the case of UK's retailing group Sainsbury's. Sainsbury's has a network of primary consolidation centres, which are shared-user storage warehouses. Stocks are consolidated between suppliers, but remain the supplier's property until the goods are delivered at Sainsbury's. This approach results in inventory holding costs that are the same for all suppliers.

\subsubsection{A two-period problem}

In this section we consider the following scenario for the capacitated case. We assume

- $T=2$,

- $D=(1,1)$,

- $h_{s}=h$, for all $s \in \mathcal{S}$, 
- $f_{s t}=f_{t}$, for all $s \in \mathcal{S}$ and all $t=1, \ldots, T$.

(We may observe that the problem instance described in Example 2.3.2 fulfills these conditions.) Let $x$ be an instance of this problem, i.e. being an array of all its parameters. Let $\pi^{C}(x)$ denote the procurement costs if the combinatorial auction is applied on $x$, and $\pi^{S}(x)$ the total procurement costs if separate auctions are applied on $x$. Recall from equation (2.1) that $\pi^{C}(x)$ boils down to

$$
C_{\mathcal{S} \backslash\left\{s_{1}\right\}}+C_{\mathcal{S} \backslash\left\{s_{2}\right\}}-C_{\mathcal{S}}
$$

if there are two winners $s_{1}$ and $s_{2}$, and to

$$
C_{\mathcal{S} \backslash\left\{s_{1}\right\}}
$$

if there is only one winner $s_{1}$. Without loss of generality we assume in the following that $s_{1}$ is the winner of (at least) $D_{1}$, implying that $s_{2}$ denotes the winner of $D_{2}$ if there is a second winner.

In the following, we will derive conditions under which the combinatorial auction is at least as good as the series of separate separate ones. As we will see, this depends on the timing of production, the capacity of the winning suppliers, and the number of winners.

Proposition 3.3.1 We have that $\pi^{C}(x) \leq \pi^{S}(x)$ in the following cases:

(i) the combinatorial auction has one winner, or

(ii) the combinatorial auction has two winners, where the second winner produces in the second period, or

(iii) the combinatorial auction has two winners, both producing in the first period, where the second winner has no capacity restrictions in this period.

\section{Proof:}

From (2.5), we know that the total procurement cost for the two separate auctions is equal to $\left(f_{1}+p_{\cdot 1}\right)^{(2)}+\left(f_{2}+p_{\cdot 2}\right)^{(2)}$. Moreover, we know that for any $n \geq 1,\left(f_{1}+p_{\cdot 1}\right)^{(n)}$ is an upper bound on the total production costs faced when producing $D_{t}$ in period $t$. We treat the three cases separately:

Part (i): Recall that the combinatorial auction payment for a single winner $s_{1}$ is equal to $C_{\mathcal{S} \backslash\left\{s_{1}\right\}}$. Excluding this single winner means that either the capacity corresponding to $\left(f_{1}+p_{\cdot 1}\right)^{(1)}$ or $\left(f_{1}+p_{\cdot 1}\right)^{(2)}$, and the capacity corresponding to $\left(f_{2}+p_{\cdot 2}\right)^{(1)}$ or $\left(f_{2}+p_{\cdot 2}\right)^{(2)}$ is still available. This means that $C_{\mathcal{S} \backslash\left\{s_{1}\right\}} \leq\left(f_{1}+p_{\cdot 1}\right)^{(2)}+\left(f_{2}+p_{\cdot 2}\right)^{(2)}$. 
Part (ii): In this case $D_{1}$ is produced by the cheapest supplier in period 1 and $D_{2}$ is produced by the cheapest supplier in period 2 . Therefore, the production plan is the same as for the series of separate auctions. Consequently, we can apply Proposition 2.3.5, which yields the desired result.

Part (iii): Recall that in this case $D_{2}$ is produced in period 1, then we have:

$$
\begin{aligned}
& C_{\mathcal{S}}=\left(f_{1}+p_{\cdot 1}\right)^{(1)}+\left(f_{1}+p_{\cdot 1}\right)^{(2)}+h, \\
& C_{\mathcal{S} \backslash\left\{s_{1}\right\}} \leq 2\left(f_{1}+p_{\cdot 1}\right)^{(2)}+h \text { since } s_{2} \text { has no capacity restrictions in period } 1 \text {, and } \\
& C_{\mathcal{S} \backslash\left\{s_{2}\right\}} \leq\left(f_{1}+p_{\cdot 1}\right)^{(1)}+\min _{s \neq s_{2}}\left(f_{2}+p_{s 2}\right) .
\end{aligned}
$$

Using (3.2), the total payment in the combinatorial auction is equal to

$$
\left(f_{1}+p_{\cdot 1}\right)^{(2)}+\min _{s \neq s_{2}}\left(f_{2}+p_{s 2}\right) \leq\left(f_{1}+p_{\cdot 1}\right)^{(2)}+\left(f_{2}+p_{\cdot 2}\right)^{(2)} .
$$

Concluding, in all three cases the combinatorial auction is at least as good as the two separate auctions.

Note that the same prove can be given for supplier-dependent setup costs. So, Proposition 3.3.1 also holds for general setup costs.

Corollary 3.3.2 Whenever the best and second best suppliers in period 1 have jointly a total capacity in period 1 that is at least 3, or the combinatorial auction has exactly one winner, we have $\pi^{C}(x) \leq \pi^{S}(x)$.

\section{Proof:}

If the combinatorial auction has exactly one winner, we have an instance of case (i) of Proposition 3.3.1. Otherwise, $D_{2}$ will be produced in period 2 and we have an instance of case (ii) of the same proposition, or $D_{2}$ will be produced in period 1 and we have an instance of case (iii).

In the following, we assume that the production costs are independent and identicallydistributed (i.i.d.) random variables and follow a uniform distribution on the interval $[a, b]$. So we may see any instance $x$ as an observation of a random vector $X$. We show in Theorem 3.3.3 that the expected total payment by the buyer in the combinatorial auction is no greater than the sum of the buyer's expected payments in the two different separate auctions. The proof of this theorem is given in section 3.3.2.

Theorem 3.3.3 Suppose that the production costs are i.i.d. random variables that have a uniform distribution on the interval $[a, b]$. Then

$$
E\left(\pi^{C}(X)\right) \leq E\left(\pi^{S}(X)\right) .
$$




\subsubsection{Proof of nonnegative expected savings}

In this section we first present the different preliminary results that are needed for the proof of Theorem 3.3.3. In section 3.3.2.1 we have a result that allows us to restrict ourselves to the case with zero setup costs. In section 3.3.2.2 we delimit different regions of the (random) parameter values, and derive lower bounds on the savings of the combinatorial auction compared to the series of separate auctions. Section 3.3.2.3 presents the density functions of order statistics, and a result on density functions that are monotone. Finally, using these results, we prove Theorem 3.3.3 in section 3.3.2.4.

\subsubsection{Setup costs}

In this section we show a result that allows us to prove Theorem 3.3.3 only for problem cases with zero setup costs. The idea is that we link a problem instance with positive setups to an instance without setups, denoted by $x$ and $\tilde{x}$ respectively, and show that $\pi^{S}(x)-\pi^{C}(x) \geq \pi^{S}(\tilde{x})-\pi^{C}(\tilde{x})$, i.e. the buyer's savings are the worst for the instance without setups.

In the following the parameters and the cost functions of instance $\tilde{x}$ are marked by a tilde. Instance $\tilde{x}$ is the same problem instance as $x$, but with $\tilde{f}=0$ and $\tilde{p}_{s t} \equiv f+p_{s t}$, so in comparison the setup costs have to be paid for any unit of production. In the following theorem we link the auction payments of $x$ and $\tilde{x}$.

Theorem 3.3.4 $\pi^{C}(x) \leq \pi^{C}(\tilde{x})$ and $\pi^{S}(x)=\pi^{S}(\tilde{x})$.

\section{Proof:}

Recall that the payments for the separate auctions and the combinatorial auctions are given in (2.5), and (3.2) or (3.3). The second part of the theorem follows from the fact that no economies of scale can be attained in the separate auctions as $D=(1,1)$ and no inventory can be held. Note that for any set of players $\mathcal{K} \subseteq \mathcal{S}$ we have $C_{\mathcal{K}} \leq \tilde{C}_{\mathcal{K}}$. We consider three cases:

Case $\mathrm{A} C_{\mathcal{S}}$ has two winners.

Case B $C_{\mathcal{S}}$ has one winner, who is also a winner in $\tilde{C}_{\mathcal{S}}$.

Case $\mathbf{C} C_{\mathcal{S}}$ has one winner, who is not a winner in $\tilde{C}_{\mathcal{S}}$. 
Case A In this case no economies of scale are attained, which means that $C_{\mathcal{S}}=\tilde{C}_{\mathcal{S}}$, having the same set of winners. Comparing the total payments gives:

$$
\begin{aligned}
\tilde{C}_{\mathcal{S} \backslash\left\{s_{1}\right\}}+\tilde{C}_{\mathcal{S} \backslash\left\{s_{2}\right\}}-\tilde{C}_{\mathcal{S}}-\left[C_{\mathcal{S} \backslash\left\{s_{1}\right\}}+C_{\mathcal{S} \backslash\left\{s_{2}\right\}}-C_{\mathcal{S}}\right] \\
=\left(\tilde{C}_{\mathcal{S} \backslash\left\{s_{1}\right\}}-C_{\mathcal{S} \backslash\left\{s_{1}\right\}}\right)+\left(\tilde{C}_{\mathcal{S} \backslash\left\{s_{2}\right\}}-C_{\mathcal{S} \backslash\left\{s_{2}\right\}}\right)+0 \\
\geq 0 .
\end{aligned}
$$

Case B Let $s_{1}$ be the unique winner in $C_{\mathcal{S}}$, such that he is also a winner in $\tilde{C}_{\mathcal{S}}$. If there is no other winner in $\tilde{C}_{\mathcal{S}}$, a comparison of payments gives:

$$
\tilde{C}_{\mathcal{S} \backslash\left\{s_{1}\right\}}-C_{\mathcal{S} \backslash\left\{s_{1}\right\}} \geq 0 .
$$

If there is another winner, called $s_{2}$, in $\tilde{C}_{\mathcal{S}}$, a comparison of payments gives:

$$
\tilde{C}_{\mathcal{S} \backslash\left\{s_{1}\right\}}+\tilde{C}_{\mathcal{S} \backslash\left\{s_{2}\right\}}-\tilde{C}_{\mathcal{S}}-C_{\mathcal{S} \backslash\left\{s_{1}\right\}} \geq 0,
$$

where the inequality holds as both $\tilde{C}_{\mathcal{S} \backslash\left\{s_{2}\right\}}-\tilde{C}_{\mathcal{S}} \geq 0$ and $\tilde{C}_{\mathcal{S} \backslash\left\{s_{1}\right\}}-C_{\mathcal{S} \backslash\left\{s_{1}\right\}} \geq 0$.

Case C Let $s_{3}$ be the winner in $C_{\mathcal{S}}$. If there is only one winner $s_{1}$ in $\tilde{C}_{\mathcal{S}}$, a comparison of payments gives:

$$
\tilde{C}_{\mathcal{S} \backslash\left\{s_{1}\right\}}-C_{\mathcal{S} \backslash\left\{s_{3}\right\}} \geq 0,
$$

where the inequality follows from $\tilde{C}_{\mathcal{S} \backslash\left\{s_{1}\right\}} \geq \tilde{C}_{\mathcal{S}}=\tilde{C}_{\mathcal{S} \backslash\left\{s_{3}\right\}} \geq C_{\mathcal{S} \backslash\left\{s_{3}\right\}}$. If there are two winners $s_{1}$ and $s_{2}$ in $\tilde{C}_{\mathcal{S}}$, we have for the same reason, together with $\tilde{C}_{\mathcal{S} \backslash\left\{s_{2}\right\}}-\tilde{C}_{\mathcal{S}} \geq 0$ :

$$
\tilde{C}_{\mathcal{S} \backslash\left\{s_{1}\right\}}+\tilde{C}_{\mathcal{S} \backslash\left\{s_{2}\right\}}-\tilde{C}_{\mathcal{S}}-C_{\mathcal{S} \backslash\left\{s_{3}\right\}} \geq 0 .
$$

We conclude that $\pi^{C}(x) \leq \pi^{C}(\tilde{x})$, and the desired result follows.

In section 3.3.2.4 we show that $E\left(\pi^{C}(X)\right) \leq E\left(\pi^{S}(X)\right)$ for the case without setups. Then, it follows from Theorem 3.3.4 that introducing setup costs, i.e. giving a reduction for the second unit produced, gives an even better result.

\subsubsection{Lower bounds}

In this section we derive lower bounds on the savings from using the combinatorial auction instead of a series of separate auctions. For reasons that will become clear in section 3.3.2.4, we only consider the case where

- we have two winners in the combinatorial auction, $s_{1}$ and $s_{2}$, and 
- the cheapest and the second cheapest suppliers in period 1 have a capacity equal to 1 in period 1 .

These lower bounds depend on the (random) values of the ordered unit production costs of the suppliers. More precisely, the payments of the combinatorial auction with zero setup costs depend on the values

$$
\begin{aligned}
& r \equiv p_{1}^{(2)}+h, \\
& q \equiv p_{1}^{(3)}+h, \\
& u \equiv p_{2}^{(1)}, \text { and } \\
& v \equiv p_{2}^{(2)},
\end{aligned}
$$

where $p_{t}^{(i)}$ is the $i$-th lowest unit production cost in period $t$. By definition we have $u \leq v$ and $r \leq q$. Define the following five regions in the space of $u$ and $v$, see Figure 3.1:

(S1) $q \leq v$ and $q \leq u$,

(S2) $r \leq u \leq v \leq q$,

(S3) $r \leq u \leq q \leq v$,

(S4) $u \leq r \leq v$, where $v$ can either be smaller or larger than $q$,

(S5) $v \leq r$.

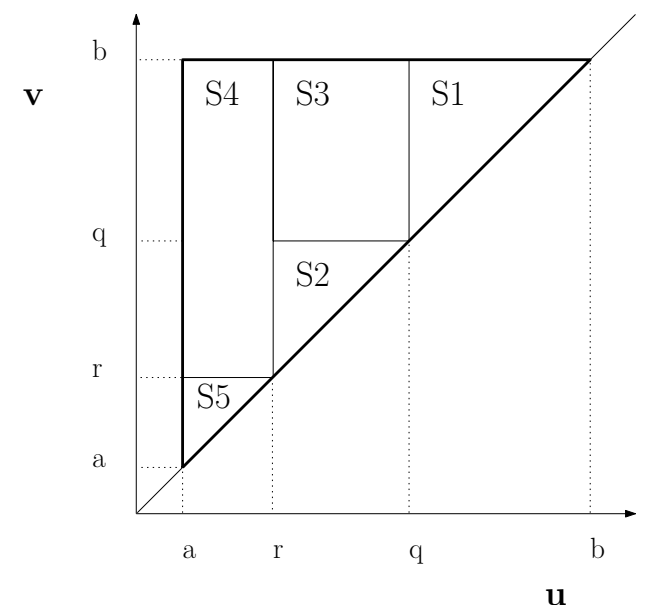

Figure 3.1: Regions (S1) to (S5) 
(Note that unlike Figure 3.1, $r$ and $q$ may be larger than $b$.) For these five regions we can find (upper bounds on) the combinatorial payments, and therefore (lower bounds for) the (possibly negative) savings of the payments of the combinatorial auction with respect to the total payments of the two separate auctions, i.e. $\pi^{S}(x)-\pi^{C}(x)$.

Lemma 3.3.5 For $f=0$ we have that

$$
\pi^{S}(x)-\pi^{C}(x) \geq \begin{cases}v-q+r-q & \text { if } x \in(S 1) \\ r-u & \text { if } x \in(S 2) \\ r-u+v-q & \text { if } x \in(S 3) \\ v-r & \text { if } x \in(S 4) \\ 0 & \text { if } x \in(S 5) .\end{cases}
$$

\section{Proof:}

Note that because the setup costs are equal to zero, we have $\pi^{S}(x)=p_{1}^{(2)}+p_{2}^{(2)}$.

Region (S1) We have that $s_{1}$ and $s_{2}$ will produce all demand in period 1 , and also without one of these players, $D_{2}$ is still produced in period 1 . Therefore,

$$
\begin{aligned}
C_{\mathcal{S}} & =p_{1}^{(1)}+p_{1}^{(2)}+h, \\
C_{\mathcal{S} \backslash\left\{s_{1}\right\}} & =p_{1}^{(2)}+p_{1}^{(3)}+h, \\
C_{\mathcal{S} \backslash\left\{s_{2}\right\}} & =p_{1}^{(1)}+p_{1}^{(3)}+h .
\end{aligned}
$$

Using (3.2), we get $\pi^{C}(x)=2 p_{1}^{(3)}+h$, and $\pi^{S}(x)-\pi^{C}(x)=p_{1}^{(2)}+p_{2}^{(2)}-2 p_{1}^{(3)}-h=$ $v-q+r-q$.

Region (S2) Again $s_{1}$ and $s_{2}$ will produce all demand in period 1, but when one of these players is not available, $D_{2}$ will be produced in period 2. In this case,

$$
\begin{aligned}
C_{\mathcal{S}} & =p_{1}^{(1)}+p_{1}^{(2)}+h, \\
C_{\mathcal{S} \backslash\left\{s_{1}\right\}} & =p_{1}^{(2)}+\min _{s \neq s_{1}} p_{s 2}, \\
C_{\mathcal{S} \backslash\left\{s_{2}\right\}} & =p_{1}^{(1)}+\min _{s \neq s_{2}} p_{s 2} .
\end{aligned}
$$

We may observe that

$$
\min _{s \neq s_{1}} p_{s 2}+\min _{s \neq s_{2}} p_{s 2} \leq p_{2}^{(1)}+p_{2}^{(2)}
$$

which means that $\pi^{C}(x)$ is at most $p_{2}^{(1)}+p_{2}^{(2)}-h$ and savings are at least $p_{1}^{(2)}+$ $p_{2}^{(2)}-p_{2}^{(1)}-p_{2}^{(2)}+h=r-u$. 
Region (S3) Again $s_{1}$ and $s_{2}$ will produce all demand in period 1, but now the production timing of $D_{2}$ is not known in advance. We then have

$$
\begin{aligned}
C_{\mathcal{S}} & =p_{1}^{(1)}+p_{1}^{(2)}+h, \\
C_{\mathcal{S} \backslash\left\{s_{1}\right\}} & =p_{1}^{(2)}+\min \left\{p_{1}^{(3)}+h, \min _{s \neq s_{1}} p_{s 2}\right\}, \\
C_{\mathcal{S} \backslash\left\{s_{2}\right\}} & =p_{1}^{(1)}+\min \left\{p_{1}^{(3)}+h, \min _{s \neq s_{2}} p_{s 2}\right\} .
\end{aligned}
$$

At least one of the minima $\min _{s \neq s_{1}} p_{s 2}$ and $\min _{s \neq s_{2}} p_{s 2}$ equals $p_{2}^{(1)}$, which is smaller than $p_{1}^{(3)}+h$. We conclude that $\pi^{C}(x)$ is at most $p_{2}^{(1)}+p_{1}^{(3)}$, and savings are at least $p_{1}^{(2)}+p_{2}^{(2)}-p_{2}^{(1)}-p_{1}^{(3)}=r-u+v-q$.

Region (S4) In this case each demand is produced by the cheapest supplier in each period, but without $s_{2}$ demand $D_{2}$ will be produced in period 1 . Therefore,

$$
\begin{aligned}
C_{\mathcal{S}} & =p_{1}^{(1)}+p_{2}^{(1)}, \\
C_{\mathcal{S} \backslash\left\{s_{1}\right\}} & =p_{1}^{(2)}+p_{2}^{(1)}, \\
C_{\mathcal{S} \backslash\left\{s_{2}\right\}} & =p_{1}^{(1)}+p_{1}^{(2)}+h .
\end{aligned}
$$

We have $\pi^{C}(x)=2 p_{1}^{(2)}+h$, and savings of $p_{1}^{(2)}+p_{2}^{(2)}-2 p_{1}^{(2)}-h=v-r$. (Note that we already know from Proposition 3.3.1 that the savings are nonnegative. As said before, we still consider this case to compensate for possible losses from regions (S1), (S2) and (S3).)

Region (S5) Again each demand is produced by the cheapest supplier in each period, but the timing does not change for the cases with one player less. Therefore,

$$
\begin{aligned}
C_{\mathcal{S}} & =p_{1}^{(1)}+p_{2}^{(1)}, \\
C_{\mathcal{S} \backslash\left\{s_{1}\right\}} & =p_{1}^{(2)}+p_{2}^{(1)}, \\
C_{\mathcal{S} \backslash\left\{s_{2}\right\}} & =p_{1}^{(1)}+p_{2}^{(2)} .
\end{aligned}
$$

This gives $\pi^{C}(x)=p_{1}^{(2)}+p_{2}^{(2)}$, the same as $\pi^{S}(x)$, thus the savings are equal to zero. 


\subsubsection{Density functions}

As we have seen, the payments in the two auctions are defined by the cheapest supplier in period 2, the second cheapest suppliers in period 1 and 2 , and the third cheapest supplier in period 1 . When the production costs are randomly generated, we need to use the corresponding order statistics. In the proof of Theorem 3.3.3 we make use of the density function of a pair of order statistics. Let $\left\{Y_{i}\right\}_{i=1}^{n}$ be i.i.d. random variables with density function $f$ and distribution function $F$. According to Rohatgi and Ehsanes Saleh [43, page 175], we have the following formula for the joint distribution of order statistics $Y_{(j)}, Y_{(k)}$, where $1 \leq j<k \leq n$ :

$$
g_{j k}\left(y_{j}, y_{k} \mid n\right)=n ! \frac{F^{j-1}\left(y_{j}\right)\left[F\left(y_{k}\right)-F\left(y_{j}\right)\right]^{k-j-1}\left[1-F\left(y_{k}\right)\right]^{n-k}}{(j-1) !(k-j-1) !(n-k) !} f\left(y_{j}\right) f\left(y_{k}\right)
$$

if $y_{j}<y_{k}$, and zero otherwise.

We will show that the expected lower bound on the savings found in Lemma 3.3.5 is always nonnegative, giving the desired result. To calculate this expected value, we need the joint density functions of $\left(p_{1}^{(2)}, p_{1}^{(3)}\right)$ and $\left(p_{2}^{(1)}, p_{2}^{(2)}\right)$, which are mutual independent. Let $n_{1} \geq 3$ be the number of suppliers having capacity in period 1 , and $n_{2} \geq 2$ the number of suppliers having capacity in period 2. From (3.4), we have that

$$
g_{12}\left(u, v \mid n_{2}\right)=\frac{n_{2}\left(n_{2}-1\right)}{(b-a)^{n_{2}}}(b-v)^{n_{2}-2}
$$

if $a \leq u<v \leq b$, and zero otherwise, and

$$
g_{23}\left(r-h, q-h \mid n_{1}\right)=\frac{n_{1} !}{\left(n_{1}-3\right) !(b-a)^{n_{1}}}(r-h-a)(b-q+h)^{n_{1}-3},
$$

if $a+h \leq r<q \leq b+h$, and zero otherwise, where we used $F(x)=\frac{x-a}{b-a}$ and $f(x)=$ $\frac{1}{b-a}$. For ease of notation, define $g_{12}\left(v \mid n_{2}\right) \equiv \frac{n_{2}\left(n_{2}-1\right)}{(b-a)^{n_{2}}}(b-v)^{n_{2}-2}$ as this expression is independent of $u$.

We will use the following observation in the proof of Theorem 3.3.3.

Observation 3.3.6 Within each period all the suppliers have the same probability to be the $i$-th cheapest one. This is a consequence of the production costs being i.i.d. random variables. It follows that the distribution function of $p_{t}^{(i)}$ equals the distribution function of $\left.p_{t}^{(i)}\right|_{s_{i}=s}$, where $s_{i}$ denotes the the identity of the $i$-th cheapest supplier. Furthermore, the similar relation holds for the joint distribution functions.

Furthermore, in the proof of Theorem 3.3.3 we use the following property. 
Proposition 3.3.7 Let $X$ be a random variable with continuous and monotone decreasing density function $f(x)$, and $R(x)$ a decreasing function over the possible values of $X$. Furthermore, let $A$ be the average value of $f(x)$ over the interval $[l, u]$, i.e. $\int_{l}^{u} f(x) d x=$ $A(u-l)$. Then

$$
\int_{l}^{u} R(x) f(x) d x \geq \int_{l}^{u} R(x) A d x
$$

\section{Proof:}

Let $x^{*} \in[l, u]$ be such that $f\left(x^{*}\right)=A$, which is well-defined as $f(\cdot)$ is monotone and continuous. Then we know that $f(x)-A \geq 0$ for $x \leq x^{*}$, and $f(x)-A \leq 0$ for $x \geq x^{*}$. We have:

$$
\begin{array}{rl}
\int_{l}^{u} & R(x) f(x) d x \\
& =\int_{l}^{u} R(x) A d x+\int_{l}^{u} R(x)(f(x)-A) d x \\
& =\int_{l}^{u} R(x) A d x+\int_{l}^{x^{*}} R(x)(f(x)-A) d x+\int_{x^{*}}^{u} R(x)(f(x)-A) d x \\
& \geq \int_{l}^{u} R(x) A d x+\int_{l}^{x^{*}} R\left(x^{*}\right)(f(x)-A) d x+\int_{x^{*}}^{u} R\left(x^{*}\right)(f(x)-A) d x \\
& =\int_{l}^{u} R(x) A d x+R\left(x^{*}\right) \int_{l}^{u}(f(x)-A) d x \\
& =\int_{l}^{u} R(x) A d x,
\end{array}
$$

where the inequality follows from $R(x)$ being a decreasing function and from the sign of $f(x)-A$ in the respective intervals.

\subsubsection{Proof of Theorem 3.3.3}

In this section we recall Theorem 3.3.3 and give its proof.

Theorem 3.3.3 Suppose that the production costs are i.i.d. and uniformly distributed. Then

$$
E\left(\pi^{C}(X)\right) \leq E\left(\pi^{S}(X)\right)
$$

\section{Proof:}

Let $\Psi_{1}$ be the set of all instances fulfilling the condition of Corollary 3.3.2. Note that this subset contains all instances having one winner that produces both units of demand in the first period. Furthermore, let $\Psi_{2}$ be the set of all other instances having only one 
winner, which is part of case (i) of Proposition 3.3.1. We know for all $x \in \Psi \equiv \Psi_{1} \cup \Psi_{2}$ that $\left.\pi^{C}(x)\right) \leq \pi^{S}(x)$. So it is sufficient to prove $E\left(\pi^{C}(X) \mid x \notin \Psi\right) \leq E\left(\pi^{S}(X) \mid x \notin \Psi\right)$. (Notice that we proved $\left.\pi^{C}(x)\right) \leq \pi^{S}(x)$ for more cases than $\Psi$, but these will be used in the calculation of the expected values to compensate for possible losses from other cases.)

Both $\Psi_{1}$ and $\Psi_{2}$ can be defined in terms of the identities of the suppliers corresponding to the order statistics. $\Psi_{1}$ links all combinations of two suppliers having at least capacity 3 in the first period to $p_{1}^{(1)}$ and $p_{1}^{(2)}$. $\Psi_{2}$ links the cheapest supplier in period 1 to $p_{2}^{(1)}$. So, using Observation 3.3.6, the conditional distribution functions for the order statistics, for all cases outside these subsets, equals the unconditional distribution. Therefore, in the following we may leave out the condition on $X$.

We prove that the expected (lower bounds on the) savings are nonnegative for any given value of $v=p_{2}^{(2)}$. We distinguish three cases:

Case $1 q \leq v \leq b$,

Case $2 r \leq v \leq q$, and

Case $3 a \leq v \leq r$.

Let $L S_{i}(r, q, v)$ denote the expected (lower bound on) savings for region $i$ and fixed values $(r, q, v)$. These are respectively:

$$
\begin{aligned}
& L S_{1}(r, q, v)=\int_{q}^{v}(v-q+r-q) g_{12}\left(u, v \mid n_{2}\right) d u=g_{12}\left(v \mid n_{2}\right)(v-q)(v-q+r-q) \\
& L S_{2}(r, q, v)=\int_{r}^{v}-(u-r) g_{12}\left(u, v \mid n_{2}\right) d u=\frac{-g_{12}\left(v \mid n_{2}\right)}{2}(v-r)^{2} \\
& L S_{3}(r, q, v)=\int_{r}^{q}(r-u+v-q) g_{12}\left(u, v \mid n_{2}\right) d u=g_{12}\left(v \mid n_{2}\right)\left((q-r)(v-q)-\frac{(q-r)^{2}}{2}\right) \\
& L S_{4}(r, q, v)=\int_{a}^{r}(v-r) g_{12}\left(u, v \mid n_{2}\right) d u=g_{12}\left(v \mid n_{2}\right)(r-a)(v-r) \\
& L S_{5}(r, q, v)=0 .
\end{aligned}
$$

Case 1 For such a value of $v$, we can see that $u$ ranges over the regions (S1), (S3), and (S4), see Figure 3.1. So, we have to add up $L S_{1}(r, q, v), L S_{3}(r, q, v)$ and $L S_{4}(r, q, v)$. This equals

$$
R(r, q, v) \equiv g_{12}\left(v \mid n_{2}\right)\left((v-q)^{2}-\frac{(q-r)^{2}}{2}+(r-a)(v-r)\right)
$$


Our aim is to show that expected value of $R(r, q, v)$ is nonnegative. Let $A(r)$ be a constant depending on the value of $r$ such that for $A(r)$ it holds that

$$
\int_{r}^{v} A(r) d q=\int_{r}^{v} g_{23}\left(r-h, q-h \mid n_{1}\right) d q .
$$

The function $R(r, q, v)$ is decreasing in $q$ for $r \leq q \leq v$. The same holds for $g_{23}(r-h, q-$ $\left.h \mid n_{1}\right)$ for the relevant values of $q \leq b+h$, while $A(r)$ is constant in $q$, but has on average the same value as $g_{23}\left(r-h, q-h \mid n_{1}\right)$, over the domain $[r, v]$, see (3.5). Therefore, we can apply Proposition 3.3.7:

$$
\begin{array}{rl}
\int_{r}^{v} & R(r, q, v) g_{23}\left(r-h, q-h \mid n_{1}\right) d q \\
& \geq \int_{r}^{v} R(r, q, v) A(r) d q \\
& \geq g_{12}\left(v \mid n_{2}\right) A(r) \int_{r}^{v}\left((v-q)^{2}-\frac{(q-r)^{2}}{2}+(r-a)(v-r)\right) d q \\
& =g_{12}\left(v \mid n_{2}\right) A(r)\left[\frac{-(v-q)^{3}}{3}-\frac{(q-r)^{3}}{6}+(r-a)(v-r) q\right]_{r}^{v} \\
& =g_{12}\left(v \mid n_{2}\right) A(r)\left(\frac{(v-r)^{3}}{3}-\frac{(v-r)^{3}}{6}+(r-a)(v-r)^{2}\right),
\end{array}
$$

which is nonnegative for $v \geq r$.

Case 2 For such a value of $v, u$ ranges over the regions (S2) and (S4), see Figure 3.1. We have to add up $L S_{2}(r, q, v)$ and $L S_{4}(r, q, v)$. This equals $g_{12}\left(v \mid n_{2}\right)\left(-\frac{1}{2}(v-r)^{2}+(r-a)(v-r)\right)$, which we denote by $R(v, r)$. Observe that, for a given $v$,

- $R(v, r)$ is a concave parabolic function of $r$,

- with roots $\frac{2 a+v}{3}$ and $v$, and

- has its maximum at $\frac{a+2 v}{3}$.

So, $R(v, r)$ is negative for $r \in\left[a+h, \frac{2 a+v}{3}\right)$ and positive for $r \in\left(\frac{2 a+v}{3}, v\right)$, see Figure 3.2. Again, our aim is to show that the expected value of $R(v, r)$ is nonnegative. We will show that for any fixed value of $v$ in Case 2, the expected value of $R(v, r)$ over $r$ is nonnegative. Note that as $r$ ranges from $a+h$ to $v$, we have that if $a+h \geq \frac{2 a+v}{3}$ the function itself is nonnegative over the whole range, and so is the expected value.

Now assume that $a+h \leq \frac{2 a+v}{3}$. Let $A(q)$ be a constant depending on $q$ such that it holds that

$$
\int_{a+h}^{v} A(q) d r=\int_{a+h}^{v} g_{23}\left(r-h, q-h \mid n_{1}\right) d r .
$$




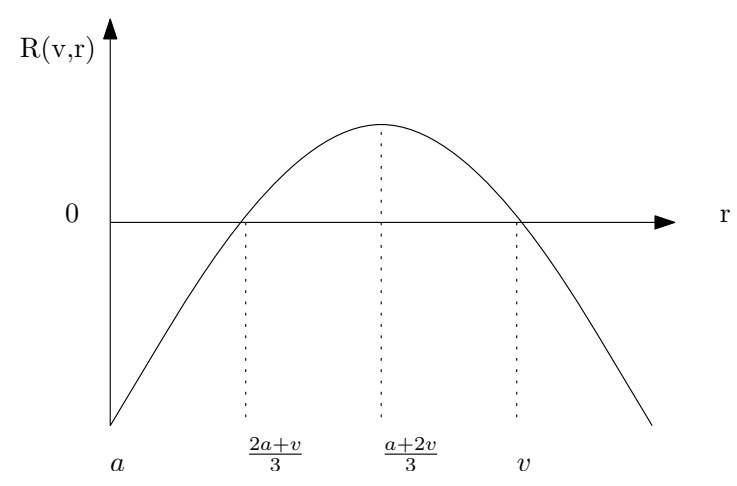

Figure 3.2: The function $R(v, r)$

Observe that for given $q, g_{23}(r-h, q-h)$ is linearly increasing in $r$, while $A(q)$ is constant in $r$. Moreover, on the interval $[a+h, v]$ the two functions intersect at $\frac{a+h+v}{2} \geq \frac{2 a+v}{3}$. Now compare $g_{23}\left(r-h, q-h \mid n_{1}\right)$ and $A(q)$ for fixed $q$ :

- For $r \in\left[a, \frac{2 a+v}{3}\right]$ we have that $A(q) \geq g_{23}(r-h, q-h)$ while $R(v, r) \leq 0$.

- For $r \in\left[\frac{2 a+v}{3}, v\right]$ we have that $R(v, r) \geq 0$ and $\int_{\frac{2 a+v}{3}}^{v} g_{23}(r-h, q-h) d r \geq A(q)$, i.e. on the interval $\left[\frac{2 a+v}{3}, v\right]$ the average value of $g_{23}\left(r-h, q-h \mid n_{1}\right)$ is at least as large as (the average value of) the constant $A(q)$. Observe that as $R(v, r)$ is a symmetric function on $\left[\frac{2 a+v}{3}, v\right]$, an integral with a linear weight function has the same value as an integral weighted by the average of this function.

So we may conclude that using $A(q)$ instead of $g_{23}\left(r-h, q-h \mid n_{1}\right)$ gives a lower bound for the expected value of $R(v, r)$. Using that $R(v, r)$ is also negative for $r \leq(a+h)$, and using a substitution $\lambda=\frac{r-a}{v-a}$ and $(1-\lambda)=\frac{v-r}{v-a}$ we get the following lower bound:

$$
\begin{aligned}
g_{12}\left(v \mid n_{2}\right) \int_{a+h}^{v}\left(-\frac{1}{2}(v-r)^{2}+(r-a)(v-r)\right) g_{23}\left(r-h, q-h \mid n_{1}\right) d r \\
\geq g_{12}\left(v \mid n_{2}\right) \int_{a+h}^{v}\left(-\frac{1}{2}(v-r)^{2}+(r-a)(v-r)\right) A(q) d r \\
\quad=g_{12}\left(v \mid n_{2}\right) A(q) \int_{a+h}^{v}\left(-\frac{1}{2}(v-r)^{2}+(r-a)(v-r)\right) d r \\
\geq g_{12}\left(v \mid n_{2}\right) A(q) \int_{a}^{v}\left(-\frac{1}{2}(v-r)^{2}+(r-a)(v-r)\right) d r \\
=g_{12}\left(v \mid n_{2}\right) A(q)(v-a)^{2} \int_{0}^{1}\left(-\frac{1}{2}(1-\lambda)^{2}+\lambda(1-\lambda)\right) d \lambda \\
=g_{12}\left(v \mid n_{2}\right) A(q)(v-a)^{2} \int_{0}^{1}\left(-\frac{3}{2} \lambda^{2}+2 \lambda-\frac{1}{2}\right) d \lambda \\
=g_{12}\left(v \mid n_{2}\right) A(q)(v-a)^{2}\left[-\frac{1}{2} \lambda^{3}+\lambda^{2}-\frac{1}{2} \lambda\right]_{0}^{1}
\end{aligned}
$$




$$
=0 \text {. }
$$

Case 3 Here we only have the savings from region (S5), see Figure 3.1, which are zero. 


\section{Chapter 4}

\section{Combinatorial Auction versus Stock Auction}

\subsection{Introduction}

In this chapter we compare the combinatorial auction and the stock auction. The stock auction allows suppliers to achieve economies of scale, but gives no flexibility in production timing. The combinatorial auction gives this flexibility and therefore decreases production costs compared to the stock auction. So, as in the case of the separate auctions in chapter 3 , it seems to be intuitive that the buyer prefers to use the combinatorial auction as this allows the suppliers to produce at lower costs. However, from Example 2.3.3 we know that, to the contrary, there are cases in which the buyer will be worse off using a combinatorial auction. As said in the previous chapter, this somewhat counterintuitive result is reminiscent of the well-known result of Hart [22], who considers the consequences in a market structure of permitting trades that were previously prohibited, where "our intuition tells us that the introduction of additional markets ought to make people better off," but provides an example in which this is not the case.

Similar to the analysis in the previous chapter, there are different effects that interfere. First we have the effect of increasing supplier production efficiency, as the combinatorial auction will allow each supplier to use capacity in different periods where the stock auction restricts the suppliers to production in the first period. One part of this effect is the use of cheaper production capacity, another part is the change in inventory levels and in the players that are holding inventory. The second effect is the change in the relative importance (or power) of the suppliers. The combinatorial auction enables suppliers to use the capacities not only of the first period but also of the other periods. 
As the (relative) costs of the suppliers may differ over the periods, this also means that suppliers that are cheap in the first period, may be expensive in the other periods giving them a weaker position if the combinatorial auction is used instead of the stock auction.

As suppliers may use the capacities from other periods in which they may be able to produce at possibly lower or higher costs, their order of importance might change. Unlike the effect of production efficiency, this might as well be advantageous as disadvantageous for the buyer.

The two effects can easily be linked to the VCG formula of the procurement costs of the stock auction. Recall formula (2.6):

$$
\pi^{I}=C_{\mathcal{S}}^{I}+\sum_{s \in \mathcal{S}}\left(C_{\mathcal{S} \backslash\{s\}}^{I}-C_{\mathcal{S}}^{I}\right)+\sum_{t=2}^{T}(t-1) H D_{t} .
$$

The efficiency effect corresponds to the change in the first and last term, $C_{\mathcal{S}}^{I}$ and $\sum_{t=2}^{T}(t-1) H D_{t}$. The effect in change in power corresponds to the change in the marginal products of the suppliers, i.e. $\sum_{s \in \mathcal{S}}\left(C_{\mathcal{S} \backslash\{s\}}^{I}-C_{\mathcal{S}}^{I}\right)$.

In section 4.2 we show that a key factor in making an example like Example 2.3.3 is supplier production capacity. We prove that, when suppliers are not restricted by capacities, the buyer will always do at least as well in the combinatorial auction as compared to the stock auction, i.e., it will not increase its total cost and might lower it. (Again, there is the analogy with Hart [22], where Hart points out that if enough new markets are opened to make the market structure complete, then his counter-intuitive result cannot occur.)

In section 4.3 we delimit for two-period setting some capacitated cases for which the combinatorial auction is the cheapest solution for the buyer. Thereafter, we show two different lower bounds on the possible loss of using the combinatorial auction instead of the stock auction.

\subsection{Uncapacitated case}

In this section we assume that the capacities of the suppliers are not binding, i.e. $R_{s t} \geq$ $\sum_{\tau=t}^{T} D_{\tau}$, for all $t \in\{1, \ldots, T\}$ and $s \in \mathcal{S}$. Moreover, $f_{s t}=f_{t}$, for all $s \in \mathcal{S}$ and all $t \in\{1, \ldots, T\}$. Under this scenario, we can show that the combinatorial auction results in a cost to the buyer that is less than or equal to that which would result under the stock auction.

Theorem 4.2.1 If the capacities are not binding, we have $\pi^{C} \leq \pi^{I}$. 


\section{Proof:}

Consider the optimal allocation of the combinatorial auction and let $T_{s} \subseteq\{1, \ldots, T\}$ be the index set of demands won by $s \in \mathcal{S}$ and $C_{\mathcal{S}}^{s}$ is the costs of supplier $s$ when delivering these demands, i.e., $C_{\mathcal{S}}=\sum_{s \in \mathcal{S}} C_{\mathcal{S}}^{s}$. Recall that $p_{t}^{(i)}$ denotes the $i$-th lowest unit production cost in period $t$.

As the capacities are not binding, we know that the stock auction has only one winner. Moreover, because the setup costs are the same for all suppliers, the winner of the stock auction will be the one with the cheapest unit production costs in period 1 . This supplier will obviously be also the winner for demand $D_{1}$ (and maybe others) in the combinatorial auction. Finally the payments of the stock auction will be determined by the supplier with the second cheapest unit production costs in period 1 . Therefore,

$$
\begin{aligned}
\pi^{I}-\pi^{C} & =C_{\mathcal{S} \backslash\left\{s_{1}\right\}}^{I}+\sum_{t=2}^{T}(t-1) H D_{t}-\left[C_{\mathcal{S}}+\sum_{s \in \mathcal{S}}\left(C_{\mathcal{S} \backslash\{s\}}-C_{\mathcal{S}}\right)\right] \\
& =f_{1}+p_{1}^{(2)} \sum_{t=1}^{T} D_{t}+\sum_{t=2}^{T}(t-1) H D_{t}-\left[C_{\mathcal{S}}+\sum_{s \in \mathcal{S}}\left(C_{\mathcal{S} \backslash\{s\}}-C_{\mathcal{S}}\right)\right] \\
& =f_{1}+\sum_{s \in \mathcal{S}} \sum_{t \in T_{s}}\left(p_{1}^{(2)}+(t-1) H\right) D_{t}-\left[\sum_{s \in \mathcal{S}} C_{\mathcal{S}}^{s}+\sum_{s \in \mathcal{S}}\left(C_{\mathcal{S} \backslash\{s\}}-C_{\mathcal{S}}\right)\right] \\
& \left.=f_{1}+\sum_{s \in \mathcal{S}}\left[\sum_{t \in T_{s}}\left(p_{1}^{(2)}+(t-1) H\right) D_{t}+\left(C_{\mathcal{S}}-C_{\mathcal{S}}^{s}\right)-C_{\mathcal{S} \backslash\{s\}}\right)\right] .
\end{aligned}
$$

To show the desired inequality it is enough to prove that

$$
\begin{aligned}
f_{1}+\sum_{t \in T_{s_{1}}}\left(p_{1}^{(2)}+(t-1) H\right) D_{t}+\left(C_{\mathcal{S}}-C_{\mathcal{S}}^{s_{1}}\right) & \geq C_{\mathcal{S} \backslash\left\{s_{1}\right\}}, \text { and } \\
\sum_{t \in T_{s}}\left(p_{1}^{(2)}+\sum_{\tau=1}^{t-1} h_{\tau}\right) D_{t}+\left(C_{\mathcal{S}}-C_{\mathcal{S}}^{s}\right) & \geq C_{\mathcal{S} \backslash\{s\}}, \quad s \in \mathcal{S} \backslash\left\{s_{1}\right\},
\end{aligned}
$$

where $s_{1}$ is the winning supplier for $D_{1}$ in the combinatorial auction. Note that if $s$ is not a winner that $T_{s}=\varnothing, C_{\mathcal{S}}^{s}=0$ and $C_{\mathcal{S} \backslash\{s\}}=C_{\mathcal{S}}$, which reduces (4.2) to $C_{\mathcal{S}} \geq C_{\mathcal{S}}$, which holds trivially. Therefore, we may assume that $s$ is a winning supplier in the combinatorial auction. For any winning supplier $s$ we can easily find an upper bound for $C_{\mathcal{S} \backslash\{s\}}$ as follows: For the suppliers in $\mathcal{S} \backslash\{s\}$ we just use the production plan from $C_{\mathcal{S}}$, which part of the production plan has cost $C_{\mathcal{S}}-C_{\mathcal{S}}^{s}$. Furthermore, we need a feasible reallocation of the demand that was assigned to $s$ : Inequality (4.1) holds by observing that $s_{1}$ is the cheapest supplier in period 1 , therefore in the absence of $s_{1}$ the demands allocated to $s_{1}$ can be produced by the second cheapest supplier in period 1. To show inequality (4.2), we may observe that $s_{1} \in \mathcal{S} \backslash\{s\}$ and therefore 
$s_{1}$ can produce the demands assigned to $s$ incurring additional costs that sum up to $\sum_{t \in T_{s}}\left(p_{1}^{(1)}+(t-1) H\right) D_{t} \leq \sum_{t \in T_{s}}\left(p_{1}^{(2)}+(t-1) H\right) D_{t}$. Note that here we do not have to add an additional setup cost as we know that supplier $s_{1}$ already produces some other demands. We can conclude that both inequalities hold, and therefore the desired result follows.

In Example 2.3.3 we have shown that Theorem 4.2.1 does not hold, in general, in the capacitated case. However, for a two-period case, we can bound the possible loss from using the combinatorial auction instead of the stock auction. In section 4.3.2 we show that the possible loss can be bounded by one setup cost. In section 4.3.3 we show that the loss is also limited by the difference in unit production costs of the best two suppliers, i.e. the more competition between suppliers, the less risk we bear from using the combinatorial auction.

\subsection{Capacitated case}

So far we have seen that the buyer always prefers the combinatorial auction over the stock auction in the uncapacitated case. However, Example 2.3.3 showed that the stock auction might be cheaper in the capacitated setting. In this section we will have a closer look at the capacitated case by considering a two-period scenario with the same setup costs and the same inventory holding costs across all the suppliers.

First, we delimit a set of problem instances for which the combinatorial auction is never more expensive than the stock auction. Second, we show a lower bound on the savings, which show that the stock auction can only be better by one setup cost. Finally, we define a lower bound on the savings that reflects the competition in the stock auction.

\subsubsection{A two-period problem}

In the rest of this section, we assume that

- $T=2$,

- $h_{s}=h$, for all $s \in \mathcal{S}$,

- $f_{s t}=f$, for all $s \in \mathcal{S}$ and all $t=1, \ldots, T$. 
Note that the problem instance described in Example 2.3.3 fulfills these conditions. The setting is similar to the setting in section 3.3.1 but here we handle a general demand vector $D$ and stationary setup costs. We need a further specification in our notation for unit production costs. Denote by $\left.p_{t}^{(i)}\right|_{\alpha}$ the $i$-th cheapest unit production costs among all those suppliers who have enough capacity to produce $\alpha$ units in period $t$. Recall that from equation (2.1) it follows that the VCG payment in the combinatorial auction boils down to $C_{\mathcal{S} \backslash\left\{s_{1}\right\}}+C_{\mathcal{S} \backslash\left\{s_{2}\right\}}-C_{\mathcal{S}}$ if there are two winners $s_{1}$ and $s_{2}$, and to $C_{\mathcal{S} \backslash\left\{s_{1}\right\}}$ if there is only one winner $s_{1}$. For any number of winners, we assume that $s_{1}$ is the winner of (at least) $D_{1}$. Similar expressions hold for the stock auction, where the costs are indicated with an index $I$ and the winners are denoted by $w_{1}$ and $w_{2}$. Since in the stock auction the buyer holds inventory, the total procurement costs are

$$
\pi^{I}= \begin{cases}C_{\mathcal{S} \backslash\left\{w_{1}\right\}}^{I}+C_{\mathcal{S} \backslash\left\{w_{2}\right\}}^{I}-C_{\mathcal{S}}^{I}+H D_{2} & \text { for two winners } \\ C_{\mathcal{S} \backslash\left\{w_{1}\right\}}^{I}+H D_{2} & \text { for one winner. }\end{cases}
$$

In the following, we present a couple of remarks that will be used when showing the relationship between $\pi^{C}$ and $\pi^{I}$. First, the combinatorial auction has a choice between producing all demand in period 1 and producing demand just-in-time. If all demand is produced in the first period the production plan is the same as in the stock auction as a consequence of the cost minimizing property of the VCG mechanism. (Note, however, that the inventories are hold at different levels at possibly different costs.) The other way around we have:

Remark 4.3.1 Whenever the optimal allocation of the combinatorial auction differs from the stock auction, we know that the combinatorial auction produces $D_{2}$ in period 2.

When the stock auction has two winners we have that $D_{1}$ is produced at a unit cost of $\left.p_{1}^{(2)}\right|_{D_{1}}$ when $D_{1}<D_{2}$ and $\left.p_{1}^{(1)}\right|_{D_{1}}=\left.p_{1}^{(1)}\right|_{D_{2}}$, or at a unit cost of $\left.p_{1}^{(1)}\right|_{D_{1}}$, otherwise. As a consequence the following remark holds:

Remark 4.3.2 If the stock auction has two winners, the cheapest supplier among all those who can produce $D_{1}$ in period 1 is one of the winners in the stock auction.

Finally, there is a straightforward bound for the marginal contribution of the two winning suppliers.

Remark 4.3.3 When the combinatorial auction has two winners where $s_{2}$ produces $D_{2}$ in period 2, we have:

$$
C_{\mathcal{S} \backslash\left\{s_{t}\right\}}-C_{\mathcal{S}} \leq\left(\left.p_{t}^{(2)}\right|_{D_{t}}-\left.p_{t}^{(1)}\right|_{D_{t}}\right) D_{t} \text { for } t=1,2
$$


Now we derive conditions under which the combinatorial auction is at least as good as the stock auction, depending on the number of winners in both auctions.

Proposition 4.3.4 We have that $\pi^{C} \leq \pi^{I}$ in the following cases:

(i) the combinatorial auction has one winner, or

(ii) the combinatorial auction has two winners, while the stock auction has only one winner and $w_{1}=s_{1}$, or

(iii) both auctions have two winners and their optimal allocations are the same.

\section{Proof:}

Part (i): First suppose that the stock auction has also exactly one winner, then the savings can be written as

$$
\begin{aligned}
\pi^{I}-\pi^{C} & =C_{\mathcal{S} \backslash\left\{w_{1}\right\}}^{I}+H D_{2}-C_{\mathcal{S} \backslash\left\{s_{1}\right\}} \\
& \geq C_{\mathcal{S} \backslash\left\{w_{1}\right\}}^{I}+H D_{2}-\left(C_{\mathcal{S} \backslash\left\{s_{1}\right\}}^{I}+H D_{2}\right) \\
& =C_{\mathcal{S} \backslash\left\{w_{1}\right\}}^{I}-C_{\mathcal{S} \backslash\left\{s_{1}\right\}}^{I},
\end{aligned}
$$

where we use the fact that all allocations in the stock auction are also feasible for the combinatorial auction. Now it is easy to see that $C_{\mathcal{S} \backslash\left\{w_{1}\right\}}^{I}-C_{\mathcal{S} \backslash\left\{s_{1}\right\}}^{I}$ is a nonnegative number. If $s_{1}=w_{1}$, then this difference is equal to zero. Otherwise, $C_{\mathcal{S} \backslash\left\{s_{1}\right\}}^{I}=C_{\mathcal{S}}^{I}$, since the winner of the stock auction $w_{1}$ belongs to the set $\mathcal{S} \backslash\left\{s_{1}\right\}$, implying that $C_{\mathcal{S} \backslash\left\{w_{1}\right\}}^{I}-C_{\mathcal{S} \backslash\left\{s_{1}\right\}}^{I} \geq 0$.

Now suppose that the stock auction has two winners. Because the allocations do not coincide, we know from Remark 4.3.1 that the combinatorial auction produces demand $D_{2}$ in period 2, and therefore, $D_{1}$ is made by the cheapest supplier that can produce $D_{1}$. Now, using Remark 4.3.2, we conclude that either $s_{1}=w_{1}$ or $s_{1}=w_{2}$. Without loss of generality assume that $s_{1}=w_{1}$. We have that under this assumption the savings can be written as

$$
\begin{aligned}
\pi^{I}-\pi^{C} & =C_{\mathcal{S} \backslash\left\{w_{1}\right\}}^{I}+C_{\mathcal{S} \backslash\left\{w_{2}\right\}}^{I}-C_{\mathcal{S}}^{I}+H D_{2}-C_{\mathcal{S} \backslash\left\{s_{1}\right\}} \\
& =C_{\mathcal{S} \backslash\left\{s_{1}\right\}}^{I}+C_{\mathcal{S} \backslash\left\{w_{2}\right\}}^{I}-C_{\mathcal{S}}^{I}+H D_{2}-C_{\mathcal{S} \backslash\left\{s_{1}\right\}} \\
& =\left(C_{\mathcal{S} \backslash\left\{s_{1}\right\}}^{I}+H D_{2}-C_{\mathcal{S} \backslash\left\{s_{1}\right\}}\right)+\left(C_{\mathcal{S} \backslash\left\{w_{2}\right\}}^{I}-C_{\mathcal{S}}^{I}\right),
\end{aligned}
$$

which is nonnegative since both terms in the summation are nonnegative.

Part (ii): From Remark 4.3.1 we know that $s_{2}$ produces $D_{2}$ in period 2. As a consequence $s_{1}$ is the cheapest supplier that can supply $D_{1}$, and as $w_{1}=s_{1}$ we have $\left.p_{1}^{(1)}\right|_{D_{1}}=\left.p_{1}^{(1)}\right|_{D_{1}+D_{2}}$. 
The expression of the savings is equal to

$$
\begin{aligned}
\pi^{I}-\pi^{C} & =C_{\mathcal{S} \backslash\left\{s_{1}\right\}}^{I}+H D_{2}-C_{\mathcal{S} \backslash\left\{s_{1}\right\}}-C_{\mathcal{S} \backslash\left\{s_{2}\right\}}+C_{\mathcal{S}} \\
& =\left(C_{\mathcal{S} \backslash\left\{s_{1}\right\}}^{I}+H D_{2}-C_{\mathcal{S} \backslash\left\{s_{2}\right\}}\right)+\left(C_{\mathcal{S}}-C_{\mathcal{S} \backslash\left\{s_{1}\right\}}\right) .
\end{aligned}
$$

By Remark 4.3.3 we know that $C_{\mathcal{S} \backslash\left\{s_{1}\right\}}-C_{\mathcal{S}} \leq\left(p_{1}^{(2)}\left|{ }_{D_{1}}-p_{1}^{(1)}\right|_{D_{1}}\right) D_{1}$. Therefore, it is sufficient to show that

$$
C_{\mathcal{S} \backslash\left\{s_{1}\right\}}^{I}+H D_{2}-C_{\mathcal{S} \backslash\left\{s_{2}\right\}} \geq\left(\left.p_{1}^{(2)}\right|_{D_{1}}-\left.p_{1}^{(1)}\right|_{D_{1}}\right) D_{1} .
$$

Note that $C_{\mathcal{S} \backslash\left\{s_{2}\right\}} \leq C_{\mathcal{S} \backslash\left\{s_{2}\right\}}^{I}+H D_{2}=C_{\mathcal{S}}^{I}+H D_{2}$, where the equality holds as $w_{1}$ belongs to $\mathcal{S} \backslash\left\{s_{2}\right\}$. Moreover, $C_{\mathcal{S}}^{I}=f+\left.p_{1}^{(1)}\right|_{D_{1}+D_{2}}\left(D_{1}+D_{2}\right)$, as there is only one winner. So (4.3) is implied by

$$
C_{\mathcal{S} \backslash\left\{s_{1}\right\}}^{I}-\left(f+\left.p_{1}^{(1)}\right|_{D_{1}+D_{2}}\left(D_{1}+D_{2}\right)\right) \geq\left(\left.p_{1}^{(2)}\right|_{D_{1}}-\left.p_{1}^{(1)}\right|_{D_{1}}\right) D_{1} .
$$

We distinguish two cases depending on the value of $\left.p_{1}^{(1)}\right|_{D_{2}}$.

First suppose that $\left.p_{1}^{(1)}\right|_{D_{2}}=\left.p_{1}^{(1)}\right|_{D_{1}}=\left.p_{1}^{(1)}\right|_{D_{1}+D_{2}}$. Then we may observe that:

$$
C_{\mathcal{S} \backslash\left\{s_{1}\right\}}^{I} \geq f+\left.p_{1}^{(2)}\right|_{D_{1}} D_{1}+\left.p_{1}^{(2)}\right|_{D_{2}} D_{2}
$$

which implies

$$
\begin{aligned}
C_{\mathcal{S} \backslash\left\{s_{1}\right\}}^{I} & -\left(f+\left.p_{1}^{(1)}\right|_{D_{1}+D_{2}}\left(D_{1}+D_{2}\right)\right) \\
& \geq\left(f+\left.p_{1}^{(2)}\right|_{D_{1}} D_{1}+\left.p_{1}^{(2)}\right|_{D_{2}} D_{2}\right)-\left(f+\left.p_{1}^{(1)}\right|_{D_{1}+D_{2}}\left(D_{1}+D_{2}\right)\right) \\
& \geq\left(\left.p_{1}^{(2)}\right|_{D_{1}}-\left.p_{1}^{(1)}\right|_{D_{1}+D_{2}}\right) D_{1}+\left(\left.p_{1}^{(2)}\right|_{D_{2}}-\left.p_{1}^{(1)}\right|_{D_{1}+D_{2}}\right) D_{2} \\
& =\left(\left.p_{1}^{(2)}\right|_{D_{1}}-\left.p_{1}^{(1)}\right|_{D_{1}}\right) D_{1}+\left(\left.p_{1}^{(2)}\right|_{D_{2}}-\left.p_{1}^{(1)}\right|_{D_{2}}\right) D_{2} \\
& \geq\left(\left.p_{1}^{(2)}\right|_{D_{1}}-\left.p_{1}^{(1)}\right|_{D_{1}}\right) D_{1},
\end{aligned}
$$

and the desired inequality follows.

Second, assume $\left.p_{1}^{(1)}\right|_{D_{2}}<\left.p_{1}^{(1)}\right|_{D_{1}}=\left.p_{1}^{(1)}\right|_{D_{1}+D_{2}}$. We have

$$
C_{\mathcal{S} \backslash\left\{s_{1}\right\}}^{I}=\min \left\{f+\left.p_{1}^{(2)}\right|_{D_{1}+D_{2}}\left(D_{1}+D_{2}\right), 2 f+\left.p_{1}^{(2)}\right|_{D_{1}} D_{1}+\left.p_{1}^{(1)}\right|_{D_{2}} D_{2}\right\} .
$$

We get a similar proof as above if the minimum equals the first term, as

$$
C_{\mathcal{S} \backslash\left\{s_{1}\right\}}^{I}=f+\left.p_{1}^{(2)}\right|_{D_{1}+D_{2}}\left(D_{1}+D_{2}\right) \geq f+\left.p_{1}^{(2)}\right|_{D_{1}} D_{1}+\left.p_{1}^{(2)}\right|_{D_{2}} D_{2} .
$$

Therefore, it remains to study what happens if $C_{\mathcal{S} \backslash\left\{s_{1}\right\}}^{I}$ equals the second term of the minimum. Here we have

$$
\begin{aligned}
C_{\mathcal{S} \backslash\left\{s_{1}\right\}}^{I} & -\left(f+\left.p_{1}^{(1)}\right|_{D_{1}+D_{2}}\left(D_{1}+D_{2}\right)\right) \\
= & \left(2 f+\left.p_{1}^{(2)}\right|_{D_{1}} D_{1}+\left.p_{1}^{(1)}\right|_{D_{2}} D_{2}\right)-\left(f+\left.p_{1}^{(1)}\right|_{D_{1}+D_{2}}\left(D_{1}+D_{2}\right)\right) \\
\quad= & \left(\left.p_{1}^{(2)}\right|_{D_{1}}-\left.p_{1}^{(1)}\right|_{D_{1}+D_{2}}\right) D_{1}+f-\left(\left.p_{1}^{(1)}\right|_{D_{1}+D_{2}}-\left.p_{1}^{(1)}\right|_{D_{2}}\right) D_{2} \\
& =\left(\left.p_{1}^{(2)}\right|_{D_{1}}-\left.p_{1}^{(1)}\right|_{D_{1}}\right) D_{1}+\left[f-\left(\left.p_{1}^{(1)}\right|_{D_{1}+D_{2}}-\left.p_{1}^{(1)}\right|_{D_{2}}\right) D_{2}\right] \\
& \geq\left(\left.p_{1}^{(2)}\right|_{D_{1}}-\left.p_{1}^{(1)}\right|_{D_{1}}\right) D_{1},
\end{aligned}
$$


where the last inequality follows from the fact that the allocation of the stock auction uses economies of scale, i.e.,

$$
C_{\mathcal{S}}^{I}=f+\left.p_{1}^{(1)}\right|_{D_{1}+D_{2}}\left(D_{1}+D_{2}\right) \leq 2 f+\left.p_{1}^{(1)}\right|_{D_{1}+D_{2}} D_{1}+\left.p_{1}^{(1)}\right|_{D_{2}} D_{2} .
$$

Part (iii): Follows from Proposition 2.3.4.

\subsubsection{Lower bound on losses: setup costs}

Example 2.3.3 illustrates that the stock auction can be better than the combinatorial auction. In the following, we will show that the possible loss from using the combinatorial auction instead of the stock auction is bounded by the setup costs.

Theorem 4.3.5 It holds that $\pi^{I}-\pi^{C} \geq-f$.

\section{Proof:}

From Proposition 4.3.4, we know that we can limit ourselves to the following cases

(i) the combinatorial auction has two winners, while the stock auction has only one winner and $w_{1} \neq s_{1}$,

(ii) both auctions have two winners but their optimal allocations differ.

In both cases, the allocation of the two auctions do not coincide. Therefore, we know by Remark 4.3.1 that $s_{2}$ produces demand $D_{2}$ in period 2 and this implies that $s_{1}$ is the cheapest supplier among all those that can produce $D_{1}$ in period 1 .

Part (i): The expression of the savings in this case is equal to

$$
\begin{aligned}
\pi^{I}-\pi^{C} & =C_{\mathcal{S} \backslash\left\{w_{1}\right\}}^{I}+H D_{2}-C_{\mathcal{S} \backslash\left\{s_{1}\right\}}-C_{\mathcal{S} \backslash\left\{s_{2}\right\}}+C_{\mathcal{S}} \\
& =\left(C_{\mathcal{S} \backslash\left\{w_{1}\right\}}+H D_{2}-C_{\mathcal{S} \backslash\left\{s_{2}\right\}}\right)+\left(C_{\mathcal{S}}-C_{\mathcal{S} \backslash\left\{s_{1}\right\}}\right) \\
& \geq\left(C_{\mathcal{S} \backslash\left\{w_{1}\right\}}-C_{\mathcal{S} \backslash\left\{s_{2}\right\}}^{I}\right)+\left(\left.p_{1}^{(1)}\right|_{D_{1}}-\left.p_{1}^{(2)}\right|_{D_{1}}\right) D_{1}
\end{aligned}
$$

where the inequality holds because $C_{\mathcal{S} \backslash\left\{s_{2}\right\}}^{I}+H D_{2} \geq C_{\mathcal{S} \backslash\left\{s_{2}\right\}}$, and because of Remark 4.3.3. Thus, since either $s_{2}=w_{1}$ or the winner of the stock auction $w_{1}$ belongs to $\mathcal{S} \backslash\left\{s_{2}\right\}$, we can conclude that $\pi^{I}-\pi^{C} \geq\left(\left.p_{1}^{(1)}\right|_{D_{1}}-\left.p_{1}^{(2)}\right|_{D_{1}}\right) D_{1}$. Therefore, it remains to show that $-\left(\left.p_{1}^{(2)}\right|_{D_{1}}-\left.p_{1}^{(1)}\right|_{D_{1}}\right) D_{1} \geq-f$. This easily follows by comparing the costs of the 
optimal stock auction allocation with the one where $D_{1}$ is produced by $s_{1}$ instead of $w_{1}$,

$$
\begin{aligned}
f+\left.p_{1}^{(1)}\right|_{D_{1}+D_{2}}\left(D_{1}+D_{2}\right)+h D_{2} & \leq 2 f+\left.p_{1}^{(1)}\right|_{D_{1}} D_{1}+\left.p_{1}^{(1)}\right|_{D_{1}+D_{2}} D_{2}+h D_{2} \\
\left.p_{1}^{(1)}\right|_{D_{1}+D_{2}} D_{1} & \leq f+\left.p_{1}^{(1)}\right|_{D_{1}} D_{1} \\
-f & \leq-\left(\left.p_{1}^{(1)}\right|_{D_{1}+D_{2}}-\left.p_{1}^{(1)}\right|_{D_{1}}\right) D_{1},
\end{aligned}
$$

and the desired inequality follows by noticing that $\left.p_{1}^{(1)}\right|_{D_{1}+D_{2}} \geq\left. p_{1}^{(2)}\right|_{D_{1}}$.

Part (ii): Recall that $s_{1}$ is the cheapest supplier among all those that can produce $D_{1}$ in period 1. Moreover, $w_{1}$ is the winner of $D_{1}$ and $w_{2}$ is the winner of $D_{2}$ in the stock auction. From Remark 4.3.2, we know that either $s_{1}=w_{1}$ or $s_{1}=w_{2}$, where the latter is true if $D_{1}<D_{2}$ and $\left.p_{1}^{(1)}\right|_{D_{1}}=\left.p_{1}^{(1)}\right|_{D_{2}}$.

First assume that $s_{1}=w_{1}$. The expression of the savings in this case is equal to

$$
\begin{aligned}
\pi^{I}-\pi^{C} & =C_{\mathcal{S} \backslash\left\{s_{1}\right\}}^{I}+C_{\mathcal{S} \backslash\left\{w_{2}\right\}}^{I}-C_{\mathcal{S}}^{I}+H D_{2}-C_{\mathcal{S} \backslash\left\{s_{1}\right\}}-C_{\mathcal{S} \backslash\left\{s_{2}\right\}}+C_{\mathcal{S}} \\
& =\left(C_{\mathcal{S} \backslash\left\{w_{2}\right\}}^{I}+H D_{2}-C_{\mathcal{S} \backslash\left\{s_{2}\right\}}\right)+\left(C_{\mathcal{S} \backslash\left\{s_{1}\right\}}^{I}-C_{\mathcal{S}}^{I}-C_{\mathcal{S} \backslash\left\{s_{1}\right\}}+C_{\mathcal{S}}\right) \\
& \geq C_{\mathcal{S} \backslash\left\{s_{1}\right\}}^{I}-C_{\mathcal{S}}^{I}-C_{\mathcal{S} \backslash\left\{s_{1}\right\}}+C_{\mathcal{S}} \\
& \geq C_{\mathcal{S} \backslash\left\{s_{1}\right\}}^{I}-C_{\mathcal{S}}^{I}-\left(\left.p_{1}^{(2)}\right|_{D_{1}}-\left.p_{1}^{(1)}\right|_{D_{1}}\right) D_{1},
\end{aligned}
$$

where the first inequality follows from the observation that $C_{\mathcal{S} \backslash\left\{w_{2}\right\}}^{I}+H D_{2} \geq C_{\mathcal{S} \backslash\left\{s_{2}\right\}}$ since either $s_{2}=w_{2}$, or both $w_{1}$ and $w_{2}$ belong to $\mathcal{S} \backslash\left\{s_{2}\right\}$. The second inequality is a consequence of Remark 4.3.3.

It remains to show that $C_{\mathcal{S} \backslash\left\{s_{1}\right\}}^{I}-C_{\mathcal{S}}^{I} \geq\left(\left.p_{1}^{(2)}\right|_{D_{1}}-\left.p_{1}^{(1)}\right|_{D_{1}}\right) D_{1}-f$. To show this, we have that

$$
C_{\mathcal{S}}^{I}=2 f+\left.p_{1}^{(1)}\right|_{D_{1}} D_{1}+\left.p_{1}^{(\ell)}\right|_{D_{2}} D_{2},
$$

with $\ell=1$ or 2 , where the latter holds if both $D_{2} \leq D_{1}$ and $\left.p_{1}^{(1)}\right|_{D_{1}}=\left.p_{1}^{(1)}\right|_{D_{2}}$. Furthermore, we have the following lower bound

$$
C_{\mathcal{S} \backslash\left\{s_{1}\right\}}^{I} \geq \min \left\{2 f+\left.p_{1}^{(2)}\right|_{D_{1}} D_{1}+\left.p_{1}^{(\ell)}\right|_{D_{2}} D_{2}, f+\left.p_{1}^{(1)}\right|_{D_{1}+D_{2}}\left(D_{1}+D_{2}\right)\right\},
$$

where the lower bound holds because we used for the first term the individual cheapest producers for $D_{1}$ and $D_{2}$, neglecting possible capacity restrictions. This means that

$$
\begin{aligned}
C_{\mathcal{S} \backslash\left\{s_{1}\right\}}^{I}-C_{\mathcal{S}}^{I} \geq & \min \left\{\left(\left.p_{1}^{(2)}\right|_{D_{1}}-\left.p_{1}^{(1)}\right|_{D_{1}}\right) D_{1},\right. \\
& \left.-f+\left(\left.p_{1}^{(1)}\right|_{D_{1}+D_{2}}-\left.p_{1}^{(1)}\right|_{D_{1}}\right) D_{1}+\left(\left.p_{1}^{(1)}\right|_{D_{1}+D_{2}}-\left.p_{1}^{(\ell)}\right|_{D_{2}}\right) D_{2}\right\} \\
\geq & \min \left\{\left(\left.p_{1}^{(2)}\right|_{D_{1}}-\left.p_{1}^{(1)}\right|_{D_{1}}\right) D_{1},\right. \\
& \left.-f+\left(\left.p_{1}^{(2)}\right|_{D_{1}}-\left.p_{1}^{(1)}\right|_{D_{1}}\right) D_{1}+\left(\left.p_{1}^{(1)}\right|_{D_{1}+D_{2}}-\left.p_{1}^{(\ell)}\right|_{D_{2}}\right) D_{2}\right\} \\
\geq & \min \left\{\left(\left.p_{1}^{(2)}\right|_{D_{1}}-\left.p_{1}^{(1)}\right|_{D_{1}}\right) D_{1},-f+\left(\left.p_{1}^{(2)}\right|_{D_{1}}-\left.p_{1}^{(1)}\right|_{D_{1}}\right) D_{1}\right\} \\
= & -f+\left(\left.p_{1}^{(2)}\right|_{D_{1}}-\left.p_{1}^{(1)}\right|_{D_{1}}\right) D_{1},
\end{aligned}
$$


where we used that $\left.p_{1}^{(1)}\right|_{D_{1}+D_{2}}>\left.p_{1}^{(1)}\right|_{D_{1}}$ as the stock auction has two winners and $\left.p_{1}^{(1)}\right|_{D_{1}}=\left.p_{1}^{(1)}\right|_{D_{2}}$ if $\ell=2$.

Second, assume that $s_{1}=w_{2}$. Recall that in this case we know that $D_{1}<D_{2}$ and $\left.p_{1}^{(1)}\right|_{D_{1}}=\left.p_{1}^{(1)}\right|_{D_{2}}$. Similarly as above,

$$
\pi^{I}-\pi^{C} \geq C_{\mathcal{S} \backslash\left\{s_{1}\right\}}^{I}-C_{\mathcal{S}}^{I}+\left(\left.p_{1}^{(1)}\right|_{D_{1}}-\left.p_{1}^{(2)}\right|_{D_{1}}\right) D_{1}
$$

Again it remains to show that $C_{\mathcal{S} \backslash\left\{s_{1}\right\}}^{I}-C_{\mathcal{S}}^{I} \geq\left(\left.p_{1}^{(2)}\right|_{D_{1}}-\left.p_{1}^{(1)}\right|_{D_{1}}\right) D_{1}-f$. We have that

$$
C_{\mathcal{S}}^{I}=2 f+\left.p_{1}^{(2)}\right|_{D_{1}} D_{1}+p_{1}^{(1)}\left|{ }_{D_{2}} D_{2}=2 f+p_{1}^{(2)}\right|_{D_{1}} D_{1}+\left.p_{1}^{(1)}\right|_{D_{1}} D_{2}
$$

and

$$
C_{\mathcal{S} \backslash\left\{s_{1}\right\}}^{I} \geq \min \left\{2 f+\left.p_{1}^{(2)}\right|_{D_{1}} D_{1}+\left.p_{1}^{(2)}\right|_{D_{2}} D_{2}, f+\left.p_{1}^{(1)}\right|_{D_{1}+D_{2}}\left(D_{1}+D_{2}\right)\right\},
$$

where the lower bound holds because we used for the first term the individual cheapest producers for $D_{1}$ and $D_{2}$, neglecting possible capacity restrictions. This means that

$$
\begin{aligned}
C_{\mathcal{S} \backslash\left\{s_{1}\right\}}^{I}-C_{\mathcal{S}}^{I} \geq & \min \left\{\left(\left.p_{1}^{(2)}\right|_{D_{1}}-\left.p_{1}^{(1)}\right|_{D_{1}}\right) D_{2},\right. \\
& \left.-f+\left(\left.p_{1}^{(1)}\right|_{D_{1}+D_{2}}-\left.p_{1}^{(2)}\right|_{D_{1}}\right) D_{1}+\left(\left.p_{1}^{(1)}\right|_{D_{1}+D_{2}}-\left.p_{1}^{(1)}\right|_{D_{1}}\right) D_{2}\right\} \\
\geq & \min \left\{\left(\left.p_{1}^{(2)}\right|_{D_{1}}-\left.p_{1}^{(1)}\right|_{D_{1}}\right) D_{1},-f+\left(\left.p_{1}^{(1)}\right|_{D_{1}+D_{2}}-\left.p_{1}^{(1)}\right|_{D_{2}}\right) D_{2}\right\} \\
\geq & \min \left\{\left(\left.p_{1}^{(2)}\right|_{D_{1}}-\left.p_{1}^{(1)}\right|_{D_{1}}\right) D_{2},-f+\left(\left.p_{1}^{(2)}\right|_{D_{1}}-\left.p_{1}^{(1)}\right|_{D_{1}}\right) D_{2}\right\} \\
= & -f+\left(\left.p_{1}^{(2)}\right|_{D_{1}}-\left.p_{1}^{(1)}\right|_{D_{1}}\right) D_{2} \\
\geq & -f+\left(\left.p_{1}^{(2)}\right|_{D_{1}}-\left.p_{1}^{(1)}\right|_{D_{1}}\right) D_{1},
\end{aligned}
$$

where we used in the first inequality that $\left.p_{1}^{(2)}\right|_{D_{1}} \leq\left. p_{1}^{(2)}\right|_{D_{2}}$ as a consequence of $D_{1}<D_{2}$.

\subsubsection{Lower bound on losses: competition}

In section 4.3.1 we showed that in several cases the combinatorial auction dominates the stock auction. For the other cases we were able to derive an upper bound on the possible losses when using the combinatorial auction instead of the stock auction. This bound is the (supplier independent) setup cost, and was derived in section 4.3.2. In this section we derive an alternative bound on the losses that is dependent on the unit production costs of the suppliers. In fact we show that the loss is limited by the difference 
in production costs of the two cheapest suppliers. As a consequence, the more suppliers the better the bound.

From Proposition 4.3.4 we know that the stock auction can only be cheaper than the combinatorial auction in the following cases:

- the combinatorial auction has two winners, while the stock auction has only one winner and $w_{1} \neq s_{1}$,

- both auctions have two winners but their optimal allocations differ.

Even for these two cases the possible loss from using the combinatorial auction instead of the stock auction is very limited. It can be bounded by the difference between the marginal product of one of the winners in the stock auction and the cost difference between the two best suppliers in the second period. Recall that in any case $s_{1}$ and $w_{1}$ denote the winners of (at least) $D_{1}$ in the combinatorial auction and the stock auction respectively.

Theorem 4.3.6 It holds that

(i) if the combinatorial auction has two winners, while the stock auction has only one winner and $w_{1} \neq s_{1}$, then

$$
\pi^{I}-\pi^{C} \geq\left(C_{\mathcal{S} \backslash\left\{w_{1}\right\}}^{I}-C_{\mathcal{S}}^{I}\right)-\left(\left.p_{2}^{(2)}\right|_{D_{2}}-\left.p_{2}^{(1)}\right|_{D_{2}}\right) D_{2}, \text { and }
$$

(ii) if both auctions have two winners and their optimal allocations are not the same, then

$$
\pi^{I}-\pi^{C} \geq \begin{cases}\left(C_{\mathcal{S} \backslash\left\{w_{2}\right\}}^{I}-C_{\mathcal{S}}^{I}\right)-\left(\left.p_{2}^{(2)}\right|_{D_{2}}-\left.p_{2}^{(1)}\right|_{D_{2}}\right) D_{2}, & \text { if } s_{1}=w_{1}, \\ \left(C_{\mathcal{S} \backslash\left\{w_{1}\right\}}^{I}-C_{\mathcal{S}}^{I}\right)-\left(\left.p_{2}^{(2)}\right|_{D_{2}}-\left.p_{2}^{(1)}\right|_{D_{2}}\right) D_{2}, & \text { otherwise. }\end{cases}
$$

Proof:

Part (i): We have that

$$
\begin{aligned}
\pi^{I}-\pi^{C} & =C_{\mathcal{S} \backslash\left\{w_{1}\right\}}^{I}+H D_{2}-C_{\mathcal{S} \backslash\left\{s_{1}\right\}}-C_{\mathcal{S} \backslash\left\{s_{2}\right\}}+C_{\mathcal{S}} \\
& =\left(C_{\mathcal{S} \backslash\left\{w_{1}\right\}}^{I}+H D_{2}-C_{\mathcal{S} \backslash\left\{s_{1}\right\}}\right)+\left(C_{\mathcal{S}}-C_{\mathcal{S} \backslash\left\{s_{2}\right\}}\right) \\
& \geq\left(C_{\mathcal{S} \backslash\left\{w_{1}\right\}}^{I}-C_{\mathcal{S} \backslash\left\{s_{1}\right\}}^{I}\right)+\left(C_{\mathcal{S}}-C_{\mathcal{S} \backslash\left\{s_{2}\right\}}\right) \\
& \geq\left(C_{\mathcal{S} \backslash\left\{w_{1}\right\}}^{I}-C_{\mathcal{S} \backslash\left\{s_{1}\right\}}^{I}\right)-\left(\left.p_{2}^{(2)}\right|_{D_{2}}-\left.p_{2}^{(1)}\right|_{D_{2}}\right) D_{2} \\
& \geq\left(C_{\mathcal{S} \backslash\left\{w_{1}\right\}}^{I}-C_{\mathcal{S}}^{I}\right)-\left(\left.p_{2}^{(2)}\right|_{D_{2}}-\left.p_{2}^{(1)}\right|_{D_{2}}\right) D_{2},
\end{aligned}
$$

where the first inequality follows from equation (2.3), implying that $C_{\mathcal{S} \backslash\left\{s_{1}\right\}}^{I}+H D_{2} \geq$ $C_{\mathcal{S} \backslash\left\{s_{1}\right\}}$. The second inequality is a consequence of Remark 4.3.3. The last inequality 
follows from the observation that the only winner of the stock auction $w_{1}$ belongs to the set $\mathcal{S} \backslash\left\{s_{1}\right\}$ because $w_{1} \neq s_{1}$.

Part (ii): Recall that $s_{1}$ is the cheapest supplier among all those that can produce $D_{1}$ in period 1. From Remark 4.3.2, we conclude that either $s_{1}=w_{1}$ or $s_{1}=w_{2}$, where the latter holds if both $D_{1}<D_{2}$ and $\left.p_{1}^{(1)}\right|_{D_{1}}=\left.p_{1}^{(1)}\right|_{D_{2}}$.

First, consider the case where $s_{1}=w_{1}$. Similarly to part (i), we have that

$$
\begin{aligned}
\pi^{I}-\pi^{C} & =C_{\mathcal{S} \backslash\left\{s_{1}\right\}}^{I}+C_{\mathcal{S} \backslash\left\{w_{2}\right\}}^{I}-C_{\mathcal{S}}^{I}+H D_{2}-C_{\mathcal{S} \backslash\left\{s_{1}\right\}}-C_{\mathcal{S} \backslash\left\{s_{2}\right\}}+C_{\mathcal{S}} \\
& =\left(C_{\mathcal{S} \backslash\left\{s_{1}\right\}}+H D_{2}-C_{\mathcal{S} \backslash\left\{s_{1}\right\}}\right)+\left(C_{\mathcal{S} \backslash\left\{w_{2}\right\}}^{I}-C_{\mathcal{S}}^{I}\right)+\left(C_{\mathcal{S}}-C_{\mathcal{S} \backslash\left\{s_{2}\right\}}\right) \\
& \geq\left(C_{\mathcal{S} \backslash\left\{w_{2}\right\}}^{I}-C_{\mathcal{S}}^{I}\right)+\left(C_{\mathcal{S}}-C_{\mathcal{S} \backslash\left\{s_{2}\right\}}\right) \\
& \geq\left(C_{\mathcal{S} \backslash\left\{w_{2}\right\}}^{I}-C_{\mathcal{S}}^{I}\right)-\left(\left.p_{2}^{(2)}\right|_{D_{2}}-\left.p_{2}^{(1)}\right|_{D_{2}}\right) D_{2},
\end{aligned}
$$

where the first inequality holds as $C_{\mathcal{S} \backslash\left\{s_{1}\right\}}^{I}+H D_{2} \geq C_{\mathcal{S} \backslash\left\{s_{1}\right\}}$, and the second follows again from Remark 4.3.3.

Second, consider the case where $s_{1}=w_{2}$. Similarly as before,

$$
\begin{aligned}
\pi^{I}-\pi^{C} & =C_{\mathcal{S} \backslash\left\{w_{1}\right\}}^{I}+C_{\mathcal{S} \backslash\left\{s_{1}\right\}}^{I}-C_{\mathcal{S}}^{I}+H D_{2}-C_{\mathcal{S} \backslash\left\{s_{1}\right\}}-C_{\mathcal{S} \backslash\left\{s_{2}\right\}}+C_{\mathcal{S}} \\
& =\left(C_{\mathcal{S} \backslash\left\{w_{1}\right\}}-C_{\mathcal{S}}^{I}\right)+\left(C_{\mathcal{S} \backslash\left\{s_{1}\right\}}^{I}+H D_{2}-C_{\mathcal{S} \backslash\left\{s_{1}\right\}}\right)+\left(C_{\mathcal{S}}-C_{\mathcal{S} \backslash\left\{s_{2}\right\}}\right) \\
& \geq\left(C_{\mathcal{S} \backslash\left\{w_{1}\right\}}^{I}-C_{\mathcal{S}}^{I}\right)+\left(C_{\mathcal{S}}-C_{\mathcal{S} \backslash\left\{s_{2}\right\}}\right) \\
& \geq\left(C_{\mathcal{S} \backslash\left\{w_{1}\right\}}^{I}-C_{\mathcal{S}}^{I}\right)-\left(\left.p_{2}^{(2)}\right|_{D_{2}}-\left.p_{2}^{(1)}\right|_{D_{2}}\right) D_{2} .
\end{aligned}
$$

We conclude that the result holds. 


\section{Chapter 5}

\section{Concavity and Substitutes in a Combinatorial Auction}

\subsection{Introduction}

In the setting of an exchange economy, auctions are very popular and fast mechanisms concerning the reallocation of goods (or items in auction design terminology). In this thesis we use the VCG auction mechanism because of its very nice theoretical properties like having dominant strategies, and yielding an efficient outcome, see Ausubel and Milgrom [1] and section 1.3.2.2. However, in the application of this mechanism there might be some problems. Ausubel and Milgrom [1] describe many problems that may arise for the simplest setting of the Vickrey auction, for example, the complexity of its implementation. This complexity is even more of a problem for combinatorial VCG auctions as the number of possible bids is exponential in the number of goods. So, combinatorial auctions might be very complex in already three ways:

- bidders have to determine an exponential number of bids,

- bidders and auctioneer have to communicate an exponential number of bids, and

- the auctioneer faces a winner determination problem with an exponential number of variables.

A way to avoid these complexities is to use an iterative auction format, like the English auction, where bidders can bid in different rounds and may select only the most appropriate bids. In some classes of problems there exist iterative auctions that are equivalent to the VCG mechanism, which means that they result in exactly the same 
transactions. For example, the English auction is equivalent to the Vickrey auction. De Vries et al. [53] discuss the conditions under which iterative auctions can be equivalent to the VCG mechanism applied to a combinatorial auction. It appears that equivalence relates to the existence of price equilibria.

Leonard [29] has shown for the classical assignment problem that the VCG mechanism is supported by any minimal price equilibrium with linear prices. In more general settings, the link between price equilibria and the VCG mechanism is missing and additional conditions are needed to recover it. Bikhchandani and Ostroy [4] and De Vries et al. [53] have studied the case where buyers bid for bundles of indivisible items. Bikhchandani and Ostroy [4] show that in the class of linear prices we may not find, in general, a price equilibrium. Therefore, they propose a richer class of prices, namely non-anonymous bundle prices. They show a result similar to Leonard [29] that hold if and only if the buyers-are-substitutes property holds. De Vries et al. [53] propose a combinatorial ascending price auction as the trading mechanism in the exchange economy. Under the stronger condition saying that the associated transferable utility game (TUgame) is concave, i.e. the characteristic funcion of the TU-game is submodular, they show that the auction terminates in VCG payments. Apart from the argument that ascending auctions need less communication, there is also some empirical evidence that ascending auctions are more useful than the VCG mechanism itself. In experimental tests of Kagel and Levin [25] it appears that in ascending auctions more players actually play the equilibrium strategy compared to the Vickrey auction. Players learn from the repetitive character of the auction and see less imaginary advantages from lying.

In this chapter we first address the assignment problem with capacities, where buyers can bid for collections of items but the valuations are linear. We show that the corresponding TU-game is concave. Moreover, we show that this problem is a special case of the models discussed by Bikhchandani and Ostroy [4] and De Vries et al. [53] and therefore their results can be applied to this setting. Specifically, this means that the VCG mechanism corresponds to a price equilibrium, and the VCG mechanism can be replaced by the primal-dual auction. However, the concavity, and even the buyersare-substitutes property, does not hold anymore when we include setup costs in the valuations of the buyers or when the items have different sizes, which we show by two counterexamples. We conclude that even for very straightforward extensions of the assignment problem we cannot ensure that the VCG mechanism corresponds to a price equilibrium. Finally, we show that it may be necessary to have non-anonymous prices in the price equilibrium that corresponds to the VCG mechanism. The results of this chapter can also be found in Lok, Romero Morales and Vermeulen [30]. 
The remainder of the chapter is organized as follows. In section 5.2 we give some basic definitions of cooperative games that are used in this chapter. In section 5.3 we introduce the assignment problem with capacities and show that the corresponding TUgame is concave. In section 5.4 we present the results of Bikhchandani and Ostroy [4] and De Vries et al. [53] and show that they apply to the assignment problem with capacities. In section 5.5 we present two extensions of the assignment problem with capacities and show that the VCG mechanism is not supported, in general, by price equilibria.

\subsection{Cooperative games}

In the following we present some notions of cooperative game theory in addition to the introduction in section 1.3 .

A transferable utility game, or TU-game, is a pair $(N, v)$ with set of players $N$ and characteristic function $v: 2^{N} \rightarrow \mathbb{R}$ with $v(\varnothing)=0$. A vector $u=\left(u_{i}\right)_{i \in N} \in \mathbb{R}^{N}$ is a core element of the game $(N, v)$ if $\sum_{i \in S} u_{i} \geq v(S)$ for all coalitions $S \subset N$, and $\sum_{i \in N} u_{i}=v(N)$.

Following Shapley [46] and Bikhchandani and Ostroy [4], we define the playersare-substitutes property as follows.

Definition 5.2.1 A coalition $B$ of players in $N$ are substitutes in the game $(N, v)$ if for all coalitions $S \subseteq B$ it holds that

$$
v(N)-v(N \backslash S) \geq \sum_{i \in S}(v(N)-v(N \backslash\{i\})) .
$$

In this chapter and in the article of Bikhchandani and Ostroy [4], the players considered are called buyers. Therefore we usually refer to this property as the buyersare-substitutes property. The buyers-are-substitutes property is related to concavity, a property that a TU-games may have:

Definition 5.2.2 The game $(N, v)$ is concave if for any two subsets $S$ and $T$ of $N$

$$
v(S)+v(T) \geq v(S \cup T)+v(S \cap T) .
$$

Notice that the concept concavity is equivalent to the submodularity concept for functions on sets, see Topkis [50]. The term concave is used for TU-games that have a submodular characteristic function. 
An equivalent characterization of concavity is given in the following, see for example Nemhauser and Wolsey [35].

Proposition 5.2.3 (Proposition 2.1 of Nemhauser and Wolsey [35, page 662]) The game $(N, v)$ with characteristic function $v: 2^{N} \rightarrow \mathbb{R}$ is concave if and only if for any set $S \subseteq N$ and $a, b \notin S$ with $a \neq b$ the following holds:

$$
v(S \cup\{a\})-v(S) \geq v(S \cup\{a, b\})-v(S \cup\{b\}) .
$$

In this chapter we will use the following proposition.

Proposition 5.2.4 If $(N, v)$ is concave, then the players in $N$ are substitutes in $(N, v)$.

\section{Proof:}

We prove that (5.1) holds for any $S \subseteq N$. This is clearly true for $S=N$, so consider an arbitrary $S \subset N$. Let $t=|N \backslash S|$ and $N \backslash S=\left\{a_{1}, \ldots, a_{t}\right\}$. Let $\tau$ be an arbitrary index in $\{1, \ldots, t\}$. By concavity of $(N, v)$ we know that

$$
v\left(N \backslash\left\{a_{1}, \ldots, a_{\tau-1}\right\}\right)-v\left(N \backslash\left\{a_{1}, \ldots, a_{\tau}\right\}\right) \geq v(N)-v\left(N \backslash\left\{a_{\tau}\right\}\right) .
$$

We can add up these inequalities for all $\tau$ in $\{1, \ldots, t\}$ :

$$
\sum_{\tau=1}^{t}\left[v\left(N \backslash\left\{a_{1}, \ldots, a_{\tau-1}\right\}\right)-v\left(N \backslash\left\{a_{1}, \ldots, a_{\tau}\right\}\right)\right] \geq \sum_{\tau=1}^{t}\left[v(N)-v\left(N \backslash\left\{a_{\tau}\right\}\right)\right] .
$$

This is equivalent to:

$$
v(N)-v(S) \geq \sum_{a \in N \backslash S}[v(N)-v(N \backslash\{a\})]
$$

and the desired result follows.

It is easy to show that for $|N| \leq 3$ concavity is equivalent to the players-are-substitutes property.

\subsection{The assignment problem with capacities is concave}

In this section we analyze the assignment problem with capacities (APC) defined as follows. Let $B$ be a set of buyers and $I$ a set of items to be allocated to the buyers. Each buyer $b \in B$ has a valuation $v_{b i} \geq 0$ for item $i \in I$, and a capacity $R_{b} \geq 0$. All items 
are assumed to have size one, so the capacity $R_{b}$ of buyer $b$ in fact indicates that buyer $b$ wants at most $R_{b}$ items.

The goal of the assignment problem with capacities is to assign the items in such a way that no capacity restriction is violated and the total valuation is maximized. The assignment problem $A P C(B, I)$ with capacities $\left(R_{b}\right)_{b \in B}$ can be formulated as follows:

$$
\max _{x} \sum_{b \in B} \sum_{i \in I} v_{b i} x_{b i}
$$

subject to:

$$
\begin{aligned}
\sum_{i \in I} x_{b i} & \leq R_{b}, \quad \forall b \in B \\
\sum_{b \in B} x_{b i} & \leq 1, \quad \forall i \in I \\
x_{b i} & \in\{0,1\}, \quad \forall b \in B, \forall i \in I,
\end{aligned}
$$

where $x_{b i}$ is equal to 1 if item $i$ is assigned to buyer $b$.

The optimal solution value of $A P C(B, I)$ is denoted by $V_{A P C}(B, I)$. Now notice that, for a set of buyers $A \subseteq B$, the problem $A P C(A, I)$ can be seen as a subproblem of $A P C(B, I)$ by adding the constraints $x_{b i}=0$ for all $i \in I$ and $b \notin A$, to $A P C(B, I)$. Thus it makes sense to consider the TU-game $\left(B, v_{A P C}\right)$ defined by $v_{A P C}(A) \equiv V_{A P C}(A, I)$ for all $A \subseteq B$. The remainder of this section is used to prove concavity of this particular TU-game. The proof is in two parts. First we will prove concavity of this game in case all capacities $R_{b}$ are equal to one, the classical assignment problem. Then we will use this partial result to prove concavity of the TU-game for the general problem.

Theorem 5.3.1 Assume that $R_{b}=1$ for all $b \in B$. Then the corresponding TU-game $\left(B, v_{A P C}\right)$ is concave.

\section{Proof:}

It suffices to prove that

$$
V_{A P C}(A \cup\{a\}, I)-V_{A P C}(A, I) \geq V_{A P C}(A \cup\{a, b\}, I)-V_{A P C}(A \cup\{b\}, I)
$$

holds for any subset $A$ of $B$ and any $a, b \notin A$ with $a \neq b$. We show this by induction to the number of elements of $A$.

Suppose that $A=\varnothing$. Obviously $V_{A P C}(\varnothing, I)=0$. Thus, for any set $I$,

$$
V_{A P C}(\{a\}, I)-V_{A P C}(\varnothing, I) \geq V_{A P C}(\{a, b\}, I)-V_{A P C}(\{b\}, I),
$$


since $V_{A P C}(\{a\}, I)+V_{A P C}(\{b\}, I) \geq V_{A P C}(\{a, b\}, I)$ easily follows from the optimality of the left hand side.

Suppose that the conditions are fulfilled for all possible coalitions $A$ in the set $B$ with $|A| \leq k$. Take a set $A$ with $|A|=k+1$. Let $a$ and $b$ be two different buyers in $B$ that are not in $A$. Take an item $i \in I$. First we show that

$$
V_{A P C}(A, I)-V_{A P C}(A, I \backslash\{i\}) \leq V_{A P C}(A \cup\{b\}, I)-V_{A P C}(A \cup\{b\}, I \backslash\{i\}) .
$$

Consider the instance $\operatorname{APC}(A, I)$ of the assignment problem. If item $i$ is not assigned to any buyer in an optimal allocation, the inequality holds trivially as the left hand side will be equal to zero. So, suppose that in an optimal allocation item $i$ is assigned to buyer $c \in A$. Then

$$
\begin{aligned}
V_{A P C}(A, I) & -V_{A P C}(A, I \backslash\{i\}) \\
& =v_{c i}+V_{A P C}(A \backslash\{c\}, I \backslash\{i\})-V_{A P C}(A, I \backslash\{i\}) \\
& \leq v_{c i}+V_{A P C}((A \cup\{b\}) \backslash\{c\}, I \backslash\{i\})-V_{A P C}(A \cup\{b\}, I \backslash\{i\}) \\
& \leq V_{A P C}(A \cup\{b\}, I)-V_{A P C}(A \cup\{b\}, I \backslash\{i\}),
\end{aligned}
$$

where the first inequality is due to the induction hypothesis.

Now consider the problem instance $A P C(A \cup\{a, b\}, I)$, and suppose that in an optimal assignment buyer $a$ gets item $j \in I$. Then, using our previous observation to get the first inequality, we have

$$
\begin{aligned}
V_{A P C}(A \cup & \{a, b\}, I)-V_{A P C}(A \cup\{b\}, I) \\
& =v_{a j}+V_{A P C}(A \cup\{b\}, I \backslash\{j\})-V_{A P C}(A \cup\{b\}, I) \\
& \leq v_{a j}+V_{A P C}(A, I \backslash\{j\})-V_{A P C}(A, I) \\
& \leq V_{A P C}(A \cup\{a\}, I)-V_{A P C}(A, I) .
\end{aligned}
$$

So in this case we found that

$$
V_{A P C}(A \cup\{a\}, I)-V_{A P C}(A, I) \geq V_{A P C}(A \cup\{a, b\}, I)-V_{A P C}(A \cup\{b\}, I) .
$$

If in an optimal allocation no item is assigned to buyer $a$, this inequality still holds as the right hand side will be equal to zero.

The theorem above is an extension of the result of Shapley [46] who proved that buyers are substitutes in the classical assignment problem, i.e., where $R_{b}=1$ for all $b \in B$. The result of Shapley follows from Theorem 5.3.1 and Proposition 5.2.4. We will now prove the concavity of the TU-game $\left(B, v_{A P C}\right)$. 
Theorem 5.3.2 The TU-game $\left(B, v_{A P C}\right)$ is concave.

\section{Proof:}

Without loss of generality, we can assume that $R_{b}$ is integer for all $b \in B$. First split each buyer $b \in B$ into $R_{b}$ buyers $b_{1}, \ldots, b_{R_{b}}$ with capacities $R_{b_{r}}=1$ and valuations $w_{b_{r}, i} \equiv v_{b i}$. Let $B^{\circ}$ denote the new set of buyers. From Theorem 5.3.1 we know that $\left(B^{\circ}, w\right)$ is concave. Let $S$ and $T$ be two coalitions in $B$ and let $S^{\circ}$ and $T^{\circ}$ be the corresponding coalitions in $B^{\circ}$. Then

$$
\begin{aligned}
v_{A P C}(S)+v_{A P C}(T) & =w\left(S^{\circ}\right)+w\left(T^{\circ}\right) \\
& \geq w\left(S^{\circ} \cup T^{\circ}\right)+w\left(S^{\circ} \cap T^{\circ}\right) \\
& =w\left((S \cup T)^{\circ}\right)+w\left((S \cap T)^{\circ}\right) \\
& =v_{A P C}(S \cup T)+v_{A P C}(S \cap T),
\end{aligned}
$$

since for any coalition $A \subset B$ the assignment problem $A P C\left(A^{\circ}, I\right)$ with unit capacities we constructed has the same objective value as $A P C(A, I)$.

Topkis [50] proved that the optimal value of the objective function of the transportation problem is a submodular function of the players' capacities, which means that the corresponding TU-game is concave. The transportation problem is an equivalent formulation to (APC) in terms of cost minimization. Our proof is easier and shorter though, and is also valid if total capacity is smaller than total demand. Furthermore, the buyersare-substitutes property, which is implied by concavity (see Proposition 5.2.4), is also implied by the gross substitutes property of the buyers' utilities. This follows from a result of Lehmann et al. [28, Theorem 5]. The utilities of the buyers are said to have the gross substitutes property if the increase in price of one item will not decrease the demand for any other item, see for example Gul and Stacchetti [19]. The buyers in problem (APC) have indeed valuations that satisfy the gross substitutes property. To see why, consider buyer $b$ who will solve the following problem for maximizing utility when $p_{i}$ is the price of item $i \in I$ :

$$
\max _{x} \sum_{i \in I}\left(v_{b i}-p_{i}\right) x_{b i}
$$

subject to:

$$
\begin{aligned}
\sum_{i \in I} x_{b i} & \leq R_{b} \\
x_{b i} & \in\{0,1\},
\end{aligned}
$$

which is a straightforward knapsack problem. The optimal solution is that buyer $b$ selects the $R_{b}$ items with highest values $v_{b i}-p_{i}$. Consequently, increasing the price of 
item $i \in I$ will only lower its own ranking according to $v_{b i}-p_{i}$, but not of any other item.

\subsection{Two applications}

The main object of study in both Bikhchandani and Ostroy [4] and De Vries et al. [53] is an exchange economy with a set $B$ of buyers and a set $I$ of (indivisible) items to be sold by a single seller indicated by $s$. (In fact this is a special case of the setting of Bikhchandani and Ostroy [4]. They initially also allowed multiple sellers.) The valuation of buyer $b$ for the set $S \subseteq I$ of items is $v_{b S} \geq 0$. Valuations are assumed to be non-decreasing, i.e., $v_{b S} \leq v_{b T}$ for all buyers $b \in B$ and all sets $S, T \subseteq I$ with $S \subseteq T$, and $v_{b \varnothing}=0$ for all buyers $b \in B$.

\subsubsection{The result of Bikhchandani and Ostroy}

The main result of Bikhchandani and Ostroy [4] in this setting provides a link between minimal prices in a price equilibrium, the outcome of the VCG mechanism in this setting, and a TU-game that can be associated with this exchange economy. These three different approaches to trade in the exchange economy will be discussed first, and then we present the result of Bikhchandani and Ostroy [4].

One approach to allocate items is to use a price equilibrium. A price equilibrium in this setting is a price vector $p=\left(p_{b S}\right)_{b \in B, S \subseteq I}$ together with a partition $T=\left(T_{b}\right)_{b \in B}$ of $I$ such that

$$
v_{b T_{b}}-p_{b T_{b}} \geq v_{b S}-p_{b S}
$$

holds for all $b \in B$ and $S \subseteq I$, and

$$
\sum_{b \in B} p_{b T_{b}} \geq \sum_{b \in B} p_{b S_{b}}
$$

holds for all partitions $\left(S_{b}\right)_{b \in B}$ of $I . T_{b}$ is interpreted as the set of items allocated to buyer $b$ in $B$. This economy is called $\mathcal{E}_{3}$ in Bikhchandani and Ostroy [4]. A price equilibrium $(p, T)$ is said to be minimal if for any other price equilibrium $(q, S)$ it holds that

$$
v_{b T_{b}}-p_{b T_{b}} \geq v_{b S_{b}}-q_{b S_{b}}
$$

for all $b \in B$. A minimal equilibrium price vector is a price vector that is part of a minimal price equilibrium. 
An alternative approach to allocate the items is to organize a combinatorial auction and use the VCG mechanism to sell the items in $I$. Given that the buyers report their valuations truthfully under this mechanism, the mechanism returns a partition $\left(T_{b}\right)_{b \in B}$ of $I$ such that

$$
\sum_{b \in B} v_{b T_{b}} \geq \sum_{b \in B} v_{b S_{b}}
$$

for any partition $\left(S_{b}\right)_{b \in B}$ of $I$, together with a payment

$$
p\left(b^{*}\right) \equiv \sum_{b \neq b^{*}} v_{b U_{b}}-\sum_{b \neq b^{*}} v_{b T_{b}}
$$

of buyer $b^{*}$ to the seller, where $\left(U_{b}\right)_{b \neq b^{*}}$ is a partition of $I$ such that

$$
\sum_{b \neq b^{*}} v_{b U_{b}} \geq \sum_{b \neq b^{*}} v_{b S_{b}}
$$

for any partition $\left(S_{b}\right)_{b \neq b^{*}}$ of $I$. This results in a net valuation of

$$
u\left(b^{*}\right) \equiv v_{b^{*} T_{b^{*}}}-p\left(b^{*}\right)=\sum_{b \in B} v_{b T_{b}}-\sum_{b \neq b^{*}} v_{b U_{b}}
$$

for buyer $b^{*}$ and a revenue of $u(s) \equiv \sum_{b \in B} p(b)$ for the seller.

A third approach for the players in the economy is to cooperate. This leads to a TU-game $\left(N, v_{B \& O}\right)$, where $N=B \cup\{s\}$, defined as follows. For a coalition $A \subseteq B$ of buyers, $v_{B \& O}(A)=0$. The value $v_{B \& O}(A \cup\{s\})$ is computed as follows. Let $\left(T_{b}\right)_{b \in A}$ denote a partition of $I$. The set of all such partitions is denoted by $\mathcal{P}(A)$. Then

$$
v_{B \& O}(A \cup\{s\}) \equiv \max \left\{\sum_{b \in A} v_{b T_{b}} \mid\left(T_{b}\right)_{b \in A} \in \mathcal{P}(A)\right\} .
$$

Theorem 6.1 of Bikhchandani and Ostroy [4] enables us to link these three different approaches to trade in the exchange economy with each other. Let $T=\left(T_{b}\right)_{b \in B}$ and $(p(b))_{b \in B}$ be the allocation and the payment vector of the VCG mechanism. A price vector $p=\left(p_{b S}\right)_{b \in B, S \subseteq I}$ is said to support the VCG mechanism if $(p, T)$ is a price equilibrium, and moreover $p(b)=p_{b T_{b}}$ holds for all buyers $b \in B$. Theorem 6.1 of Bikhchandani and Ostroy [4] now states the following.

Theorem 5.4.1 The following statements are equivalent:

(i) Buyers are substitutes in the TU-game $\left(N, v_{B \& O}\right)$.

(ii) The vector $\left(u(s), u(b)_{b \in B}\right)$ of net utilities is a core allocation of the TU-game $\left(N, v_{B \& O}\right)$.

(iii) Any minimal equilibrium price vector supports the VCG mechanism. 


\subsubsection{Application to the assignment problem with capacities}

Consider the assignment problem with capacities with buyer set $B$, item set $I$, valuations $v_{b i}$ and capacities $R_{b}$ as it is defined in Section 5.3. This problem can also be seen as an exchange economy with valuations given by

$$
v_{b S} \equiv V_{A P C}(\{b\}, S)
$$

for each buyer $b \in B$ and each set of items $S \subseteq I$. Clearly, the valuations defined in this way are non-decreasing. Thus, in the light of Theorem 5.4.1 of Bikhchandani and Ostroy [4], the question arises whether buyers are substitutes in the game $\left(N, v_{B \& O}\right)$ associated with this exchange economy. Using our results from the previous section we will now show that this is indeed the case.

Theorem 5.4.2 Buyers are substitutes in the TU-game $\left(N, v_{B \& O}\right)$.

\section{Proof:}

Notice that it suffices to prove that all players in $B$ are substitutes in the TU-game $(B, w)$ defined by

$$
w(A) \equiv \max \left\{\sum_{b \in A} v_{b T_{b}} \mid\left(T_{b}\right)_{b \in A} \in \mathcal{P}(A)\right\}
$$

for all coalitions $A \subseteq B$. Therefore

$$
w(A)=\max \left\{\sum_{b \in A} v_{b T_{b}} \mid\left(T_{b}\right)_{b \in A} \in \mathcal{P}(A)\right\}=V_{A P C}(A, I)=v_{A P C}(A),
$$

and $(B, w)$ is concave by Theorem 5.3.2. Hence, by Proposition 5.2.4, the players in $B$ are substitutes in the game $(B, w)$.

\subsubsection{The result of De Vries, Schummer and Vohra}

De Vries et al. [53] consider the same single seller exchange economy, defined in the beginning of this section, as Bikhchandani and Ostroy [4] with the extra assumption that all $v_{b S}$ are integer.

They consider a combinatorial ascending price auction, called the Primal Dual auction or PD auction for short, as the trading mechanism in the exchange economy. To define the PD auction we first need to introduce some notation. 
Given a vector of prices $p$, the most preferred sets of items for buyer $b$ are

$$
D_{b} \equiv\left\{T \subseteq I \mid v_{b T}-p_{b T} \geq v_{b S}-p_{b S} \quad \text { for all } S \subseteq I\right\} .
$$

Let $F \subseteq B$ be a set of buyers. A vector $\left(T_{b}\right)_{b \in F}$ is called feasible for $F$ if $T_{b} \in D_{b}$ for every buyer $b \in F$ and the elements of $\left(T_{b}\right)_{b \in F}$ are mutually disjoint. Let $Z(F)$ be the set of all feasible vectors for $F$. Let $Z^{*}(F)$ denote the set of all feasible vectors for $F$ such that

$$
\sum_{b \in F} p_{b T_{b}} \geq \sum_{b \in G} p_{b S_{b}}
$$

holds for every $G \subseteq B$ and every $\left(S_{b}\right)_{b \in G} \in Z(G)$. Let $Z^{*}=\bigcup_{F \subseteq B} Z^{*}(F)$. If for $F \subseteq B$ there is no $\left(T_{b}\right)_{b \in F}$ in $Z^{*}$ we say that $F$ is undersupplied. We say that $F$ is minimally undersupplied if $F$ is undersupplied, and no proper subset of $F$ is undersupplied.

Now let $B^{*}$ be the set of buyers $b \in B$ for which there exists an $S \in D_{b}$ with $v_{b S}-p_{b S}>0$. We say there is overdemand if $B^{*}$ is undersupplied.

The PD auction is an iterative procedure defined as follows.

\section{PD auction}

Step 0. Choose prices $p_{b S}=0$ for all $b \in B$ and $S \subseteq I$.

Step 1. With respect to the current prices, ask each buyer $b$ to report its most preferred sets of items, i.e., $D_{b}$.

Step 2. If overdemand holds, choose a minimally undersupplied set $A \subseteq B^{*}$ and for each $b \in A$ and each $S \in D_{b}$ increase the current price $p_{b S}$ by one unit and return to Step 1. All other prices stay the same for the moment. (This construction ensures in particular that $p_{b \emptyset}=0$ throughout the auction.)

Step 3. If there is no overdemand, choose an element $\left(T_{b}\right)_{b \in B}$ in $Z^{*}$, allocate the items in $T_{b}$ to buyer $b \in B$, and charge $b$ the current price $p_{b T_{b}}$.

Notice that we have some degree of freedom in the auction because of the freedom of choice of the minimally undersupplied sets and the final allocation in $Z^{*}$. Any choice made here yields a version of the PD auction. Nevertheless, as De Vries et al. [53] show, any PD auction constructed in this way features truthful reporting as an ex post Nash equilibrium, and in equilibrium it terminates in an efficient assignment.

For a coalition $A \subseteq B$ of buyers, define

$$
v_{P D}(A) \equiv \max \left\{\sum_{b \in A} v_{b T_{b}} \mid\left(T_{b}\right)_{b \in A} \in \mathcal{P}(A)\right\} \text {. }
$$


Theorem 4 of De Vries et al. [53] states the following.

Theorem 5.4.3 Suppose that $\left(B, v_{P D}\right)$ is concave. Then any PD auction terminates in VCG payments.

\subsubsection{Application to the assignment problem with capacities}

Again we can apply the result under consideration to the assignment problem with capacities. Using our result concerning the concavity of $\left(B, v_{A P C}\right)$ we can show the following statement in the context of the assignment problem with capacities.

Theorem 5.4.4 A PD auction applied to the assignment problem with capacities generates a price vector $p$ and an allocation $T$ that constitute a price equilibrium $(p, T)$. Moreover, $p$ supports the VCG mechanism.

\section{Proof:}

Let $p=\left(p_{b S}\right)_{b \in B, S \subseteq I}$ be the price vector generated by a certain $\mathrm{PD}$ auction, and let $T=\left(T_{b}\right)_{b \in B}$ be the corresponding final allocation. Since the PD auction terminates in an efficient allocation in equilibrium, we may assume that $T$ together with the VCG payments $(p(b))_{b \in B}$ is the outcome of the VCG mechanism.

It is clear that $v_{A P C}=v_{P D}$. Hence, by Theorem 5.3.2 and Theorem 5.4.3 we have that $p(b)=p_{b T_{b}}$ holds for all buyers $b \in B$. Thus, it remains to show that $(p, T)$ is a price equilibrium.

Since $T=\left(T_{b}\right)_{b \in B}$ is an element of $Z^{*}$, it is in particular feasible. Thus we know that $T_{b} \in D_{b}$ for each buyer $b \in B$, and hence

$$
v_{b T_{b}}-p_{b T_{b}} \geq v_{b S}-p_{b S}
$$

for all $S \subseteq I$. Finally we show that

$$
\sum_{b \in B} p_{b T_{b}} \geq \sum_{b \in B} p_{b S_{b}}
$$

holds for all partitions $\left(S_{b}\right)_{b \in B}$ of $I$. Take a partition $\left(S_{b}\right)_{b \in B}$ of $I$. Let $B^{+}$be the set of buyers $b$ for which $p_{b S_{b}}>0$. Let $b$ be a buyer in $B^{+}$. Since $p_{b S_{b}}>0$, we know that somewhere during the auction $S_{b} \in D_{b}$. However, due to the fact that valuations are integral and that all prices of sets in the demand of an undersupplied buyer increase in steps of size one, it is evident that $S_{b} \in D_{b}$ will remain true for the remaining time 
the auction runs. Thus, $\left(S_{b}\right)_{b \in B^{+}}$is a feasible vector for $B^{+}$. So, since $T=\left(T_{b}\right)_{b \in B}$ is an element of $Z^{*}$ and $p_{b S_{b}}=0$ for all $b \notin B^{+}$, we know that

$$
\sum_{b \in B} p_{b T_{b}} \geq \sum_{b \in B^{+}} p_{b S_{b}}=\sum_{b \in B} p_{b S_{b}}
$$

which completes the proof.

\subsection{Impossibilities}

In the previous section we proved that the TU-game associated with the assignment problem with capacities is concave. Therefore the buyers are substitutes in this TUgame, according to Proposition 5.2.4. Unfortunately concavity, and even the buyersare-substitutes property gets lost for other small and rather natural extensions of the assignment problem.

We also give a simple example of (APC) with a unique optimal assignment in which the VCG outcome is not supported by a price equilibrium where the prices are anonymous. Therefore, in general, the richer class of prices used by Bikhchandani and Ostroy, namely non-anonymous bundle prices, cannot be simplified for the assignment problem with capacities.

\subsubsection{Generalized assignment problem}

In this section we consider the case where each item $i \in I$ consumes an amount $\rho_{i}$ of the capacity of the buyers. This yields to the Generalized Assignment Problem (GAP) with buyer independent resource consumption. The problem reads as follows:

$$
\max \sum_{b \in B} \sum_{i \in I} v_{b i} x_{b i}
$$

subject to:

$$
\begin{aligned}
\sum_{i \in I} \rho_{i} x_{b i} & \leq R_{b}, \quad \forall b \in B, \\
\sum_{b \in B} x_{b i} & \leq 1, \quad \forall i \in I, \\
x_{b i} & \in\{0,1\}, \quad \forall b \in B, \forall i \in I .
\end{aligned}
$$


The value $v_{G A P}(A)$ for a coalition $A$ of buyers is the optimal value of the objective function in the above (GAP) with the additional constraints that $x_{b i}=0$ whenever $b \notin A$.

In the resulting TU-game $\left(B, v_{G A P}\right)$ the buyers will in general not be substitutes as the next example shows. Hence, also concavity will not hold in general.

Take buyer set $B=\{a, b, c\}$ with capacities $R_{a}=2, R_{b}=2$ and $R_{c}=1$ respectively. Take set of items $I=\{i, j, k\}$ with resource consumption $\rho_{i}=2, \rho_{j}=1$ and $\rho_{k}=1$ respectively. The buyers' valuations for the items are given in the Table 5.1.

\begin{tabular}{c|ccc} 
& $i$ & $j$ & $k$ \\
\hline$a$ & 6 & 5 & 5 \\
$b$ & 0 & 0 & 3 \\
$c$ & 0 & 3 & 0 \\
\hline
\end{tabular}

Table 5.1: Valuations for GAP

Given these valuations it is easy to see that we obtain $v_{G A P}(a, b, c)=12, v_{G A P}(a, c)=$ $v_{G A P}(a, b)=10$ and $v_{G A P}(a)=10$, which violates the substitutes condition

$$
v_{G A P}(a, b, c)-v_{G A P}(a) \geq 2 v_{G A P}(a, b, c)-v_{G A P}(a, c)-v_{G A P}(a, b) .
$$

Since for this problem instance of (GAP) the buyers-are-substitutes property does not hold, we know that the VCG mechanism is not supported by a price equilibrium. Let us illustrate this. We have that $v_{G A P}(b, c)=6$. Thus, the net utilities of buyers $a$, $b$ and $c$ are equal to $12-6=6,12-10=2$ and $12-10=2$, respectively. According to the optimal allocation, the buyers' payments are equal to 0,1 and 1 , respectively. This yields an auction revenue of 2 . As the only optimal solution assigns item $i$ to buyer $a$ and items $j$ and $k$ to buyers $c$ and $b$ respectively, we have that in equilibrium $6-p_{a\{i\}} \geq 10-p_{a\{j, k\}}$. From the VCG payments we know that $p_{a\{i\}}=0$, so $p_{a\{j, k\}} \geq 4$, which gives the auctioneer a revenue of at least $4>2$.

In a similar way, the vector of prices generated by the PD auction does not support the VCG mechanism. We will show that any PD auction will terminate with $p_{a\{j, k\}}=4$. Thus, as before, $p$ cannot be a price equilibrium. Any PD auction starts with all prices equal to zero. This gives the following initial demand sets (assuming disposability):

$$
\begin{aligned}
& D_{a}=\{\{j, k\},\{i, j, k\}\}, \\
& D_{b}=\{\{k\},\{j, k\},\{i, k\},\{i, j, k\}\}, \\
& D_{c}=\{\{j\},\{i, j\},\{j, k\},\{i, j, k\}\} .
\end{aligned}
$$


Here $B^{*}=\{a, b, c\}$ is undersupplied as buyer $a$ wants at least both $j$ and $k$, buyer $b$ wants at least item $k$ and buyer $c$ wants at least item $j$. The minimally undersupplied sets are $\{a, b\}$ and $\{a, c\}$. Suppose that we choose $\{a, b\}$. The algorithm would then raise the price of all sets in $D_{a}$ and $D_{b}$. After this first price-raise the demand sets remain the same, so we can do the same raise again, and even a third time. After the third iteration $D_{a}$ is still the same, but now $D_{b}=2^{I}$. Then the only minimally undersupplied set is $\{a, c\}$, so we raise the prices in $D_{a}$ a fourth time and also the prices in $D_{c}$. After this, $\{i\} \in D_{a}$. Because there is no overdemand anymore the algorithm will stop with $p_{a\{j, k\}}=4$. We arrive to the same conclusion if we choose $\{a, c\}$ as the undersupplied set in the first iteration.

\subsubsection{Setup costs}

The (APC) model assumes a linear valuation function for the buyers. In this section we include a setup cost $f_{b}$ in the valuation function. This results in the following mixedinteger linear program.

$$
\max \sum_{b \in B} \sum_{i \in I} v_{b i} x_{b i}-\sum_{b \in B} f_{b} y_{b}
$$

subject to:

$$
\begin{aligned}
\sum_{i \in I} x_{b i} & \leq R_{b} y_{b}, \quad \forall b \in B \\
\sum_{b \in B} x_{b i} & \leq 1, \quad \forall i \in I \\
x_{b i} & \in\{0,1\}, \quad \forall b \in B, \forall i \in I, \\
y_{b} & \in\{0,1\}, \quad \forall b \in B .
\end{aligned}
$$

Where $x_{b i}$ is defined as before and $y_{b}$ is equal to one if buyer $b$ processes at least one item. Again, for a coalition $A \subseteq B$ of buyers the valuation $v_{A P C S}(A)$ is defined as the optimal value of the objective function of the above program with the extra conditions that $x_{b i}=0$ and $y_{b}=0$ whenever $b \notin A$.

Consider the problem instance with $B=\{a, b, c\}, R_{a}=R_{b}=R_{c}=2$, and setup costs of $f_{a}=10, f_{b}=1$ and $f_{c}=1$ and set of items $I=\{i, j, k, m\}$. Valuations are given in Table 5.2. It can easily be checked that $v_{A P C S}(a, b, c)=12$ ( $a$ gets no items, $b$ gets items $i$ and $k$ and $c$ gets $j$ and $m$ ), $v_{A P C S}(a, c)=10$ ( $a$ gets $i$ and $k, c$ gets $j$ and $m$ ), $v_{A P C S}(a, b)=3$ ( $a$ gets $k$ and $m, b$ gets $i$ and $j$ ) and $v_{A P C S}(a)=2$ (a gets $k$ and $m$ ). Thus, the substitutes condition

$$
v_{A P C S}(a, b, c)-v_{A P C S}(a) \geq 2 v_{A P C S}(a, b, c)-v_{A P C S}(a, c)-v_{A P C S}(a, b)
$$




\begin{tabular}{c|cccc} 
& $i$ & $j$ & $k$ & $m$ \\
\hline$a$ & 4 & 4 & 6 & 6 \\
$b$ & 1 & 1 & 2 & 1 \\
$c$ & 1 & 1 & 1 & 10 \\
\hline
\end{tabular}

Table 5.2: Valuations for APCS

is violated.

Similarly to the previous section, we will show that the VCG mechanism is not supported by a price equilibrium and that the PD auction does not support the VCG mechanism. We have that $v_{A P C S}(b, c)=12$, so the net utilities of buyer $a, b$ and $c$ are $12-12=0,12-10=2$ and $12-3=9$, respectively. According to the optimal allocation the buyers' payments are 0,0 and 1 respectively, yielding an auction revenue of 1 . Since in the only optimal solution buyer a gets no item, we have that $p_{a\{k, m\}} \geq 2$. Therefore, the auction revenue is at least $2>1$.

In any PD auction we can also see that we will not terminate before $p_{a\{k, m\}} \geq 2$. The auction starts with all prices equal to zero. This gives the following initial demand sets (assuming disposability):

$$
\begin{aligned}
D_{a} & =\{S \subseteq B: k \in S, m \in S\} \\
D_{b} & =\{S \subseteq B: k \in S,|S|>1\} \\
D_{c} & =\{S \subseteq B: m \in S,|S|>1\} .
\end{aligned}
$$

Here $B^{*}=\{a, b, c\}$ is undersupplied as buyer $a$ wants at least items $k$ and $m$, buyer $b$ wants at least item $k$ and buyer $c$ wants at least item $m$. The minimally undersupplied sets are $\{a, b\}$ and $\{a, c\}$. Suppose that we choose $\{a, b\}$. The algorithm would then raise the price of all sets in $D_{a}$ and $D_{b}$. After this first price-raise $D_{a}$ remains the same, but $D_{b}$ becomes $\{S \subseteq B:|S|>1\}$. Now $\{a, b\}$ is not undersupplied anymore, but $\{a, c\}$ is. Therefore, after raising the prices in $D_{a}$ and $D_{c}$ we have that $p_{a\{k, m\}} \geq 2$. The same conclusion can be derived if we choose $\{a, c\}$ as the undersupplied set in the first iteration.

\subsubsection{The VCG mechanism is not supported by anonymous prices}

Leonard [29] showed for the classical assignment problem that the VCG mechanism is supported by any minimal price equilibrium vector with linear prices. Theorem 5.4.2 together with Theorem 5.4.1 shows that any minimal equilibrium price vector supports 
the VCG mechanism for the assignment problem with capacities (APC). But here the prices are not necessarily linear, and not even anonymous. We now show for (APC) that we really need non-anonymous prices to support the VCG mechanism.

Formally, a price vector $p=\left(p_{b S}\right)_{b \in B, S \subseteq I}$ is called anonymous if $p_{a S}=p_{b S}$ for all buyers $a$ and $b$ in $B$ and sets $S \subseteq I$ of items. It is called linear if for each $i \in I$ there is a price $\phi_{i}$ such that

$$
p_{b S}=\sum_{i \in S} \phi_{i}
$$

for all $b \in B$ and all $S \subseteq I$. Obviously a linear price vector is also anonymous.

Consider the instance of (APC) with $B=\{a, b\}, I=\{i, j, k\}, R_{a}=1$ and $R_{b}=2$. The buyers' valuations are given in Table 5.3.

\begin{tabular}{c|ccc} 
& $i$ & $j$ & $k$ \\
\hline$a$ & 5 & 4 & 2 \\
$b$ & 5 & 4 & 1 \\
\hline
\end{tabular}

Table 5.3: Valuations for APC

The unique optimal solution for this problem is that buyer $a$ gets item $k$, and buyer $b$ gets items $i$ and $j$. To calculate the VCG payments we also need the optimal solutions for the problems excluding one buyer at a time. Obviously, when on his own, $a$ chooses item $i$, while $b$ chooses items $i$ and $j$ when $a$ is not present. The VCG payments are therefore $p(a)=9-9=0$ and $p(b)=5-2=3$.

With these VCG payments we get the following conflicting conditions for linear prices $\phi_{i}, \phi_{j}$ and $\phi_{k}$ that form a price equilibrium with the allocation that assigns $k$ to $a$ and $i$ and $j$ to $b$. From the coincidence of the equilibrium prices with the VCG payments we get that

$$
\phi_{k}=0 \text { and } \phi_{i}+\phi_{j}=3 .
$$

From the equilibrium conditions we get that

$$
5-\phi_{i} \leq 2-\phi_{k} \text { and } 4-\phi_{j} \leq 2-\phi_{k}
$$

Using $\phi_{k}=0$, we have that $\phi_{i} \geq 3$ and $\phi_{j} \geq 2$. These conditions are clearly conflicting with $\phi_{i}+\phi_{j}=3$. From this we conclude that prices that support the VCG mechanism are necessarily nonlinear.

Now we also show that the prices cannot be anonymous. Let $\phi_{i j}, \phi_{i k}$ and $\phi_{j k}$ be the prices of the packages $\{i, j\},\{i, k\}$ and $\{j, k\}$ respectively. By disposability buyer $a$ has 
valuations 5,5 and 4 for these packages. From the equilibrium conditions we therefore have that

$$
5-\phi_{i j} \leq 2-\phi_{k} \text { and } 5-\phi_{i k} \leq 2-\phi_{k} \text { and } 4-\phi_{j k} \leq 2-\phi_{k} \text {. }
$$

So together with the conditions above we have that $\phi_{i} \geq 3, \phi_{j} \geq 2, \phi_{k}=0, \phi_{i j} \geq 3$, $\phi_{i k} \geq 3$ and $\phi_{j k} \geq 2$. From the perspective of the auctioneer these prices mean that the revenue from the auction is at least 5 by assigning for example $i$ to buyer $a$ and $j$ and $k$ to buyer $b$. This contradicts with the VCG outcome in which the auction has a revenue of 3. 


\section{Chapter 6}

\section{Parametric Shortest Path Tree Problem}

\subsection{Introduction}

In this chapter we present a parametric shortest path tree problem. The structure of this problem relates to the bargaining model of Sucky [49]. He considers a supply chain setting with one buyer and one supplier, where the supplier takes the first step towards a coordinated production planning, see section 1.4.2. This is a screening problem where the first player designs the rules of the game such that the second player faces incentive compatibility and individual rationality, see section 1.3.2. The parametric shortest path tree problem is a very basic, but still challenging, version of this problem.

In section 6.2 the parametric shortest path tree problem is presented as a generalization of the shortest path problem. Furthermore, it is shown that although the parametric problem is nonlinear, it can be reformulated as a linear programming problem by using duality theory, see for example Papadimitriou and Steiglitz [39]. Therefore, as this reformulation is straightforward and as linear programs are polynomially solvable, the problem itself is polynomially solvable. We are interested in applying an algorithm that uses the combinatorial structure of the problem allowing for faster optimization.

In section 6.3 we show an optimality condition for the shortest path tree problem in terms of parameter values and the set of arcs that are part of a shortest path. This condition has the nice property that it is in fact a local condition. More specifically, a solution is optimal if for all nodes the local condition holds. The optimality condition suggests a local improvement algorithm which is presented in section 6.4. Unfortunately, this local improvement algorithm is not finite, which we show in an example.

Section 6.5 presents an alternative formulation of the parametric shortest path tree 
problem. We obtain this alternative formulation by elimination of the parametric variables. The new formulation only has variables corresponding to the shortest path lengths, but still we are able to construct an example for which the local improvement algorithm is infinite. Therefore, the existence of a combinatorial algorithm that is faster than algorithms for linear programs remains an open question.

This chapter concludes with two examples that are basically parametric shortest path tree problems but with a more difficult parametrization than the problem discussed in this chapter. The first example concerns the pricing problem of a monopolist. The second example is a revenue maximization problem of an auction-designer.

\subsection{Problem formulation}

In this section we introduce the problem formulation of the parametric shortest path tree problem. We start with the standard shortest path (tree) problem. Thereafter we introduce the parametric generalization of the problem. Finally, we show that the problem can be formulated as a linear program.

\subsubsection{Shortest path problem}

Suppose we have a directed graph $G=(V, A)$ in which there is a (directed) path from source node $s$ to any other node in $V$. The length of $\operatorname{arc}(i, j) \in A$ is given by $\ell_{i j}$. The shortest path problem is to find the minimum length path from $s$ to $t$, which is only well-defined if no negative length cycles exist. For fixed source node $s$ the shortest paths to all other nodes can be found with the well known Dijkstra's algorithm in case all arc lengths are nonnegative, or the Bellman-Ford algorithm for general arc lengths, see Nemhauser and Wolsey [35]. As these shortest paths can be chosen in such a way that we have a directed tree, the problem of finding all shortest paths from $s$ is known as the shortest path tree problem, see Pallottino and Scutellà [37].

The shortest path (tree) problem can be formulated as a flow problem where one unit of flow is sent from the source to each of the other nodes. Let $f_{i j}$ be the flow on arc $(i, j)$ and $f=\left(f_{i j}\right)_{(i, j) \in A}$, then we have:

$$
\min _{f} \sum_{(i, j) \in A} \ell_{i j} f_{i j}
$$


subject to:

$$
\begin{aligned}
& \sum_{j:(j, i) \in A} f_{j i}-\sum_{j:(i, j) \in A} f_{i j}= \begin{cases}-(|V|-1), & \text { for } i=s \\
1, & \text { for } i \in V \backslash\{s\}\end{cases} \\
& f_{i j} \geq 0, \quad \forall(i, j) \in A .
\end{aligned}
$$

In this thesis we use the dual problem of (SPT):

$$
\max _{\pi} \sum_{t \in V \backslash\{s\}} \pi_{t}-(|V|-1) \pi_{s}=\sum_{t \in V \backslash\{s\}}\left(\pi_{t}-\pi_{s}\right)
$$

subject to:

(Dual-SPT)

$$
\pi_{j}-\pi_{i} \leq \ell_{i j}, \quad \forall(i, j) \in A
$$

where $\pi=\left(\pi_{i}\right)_{i \in V}$. Suppose we have a feasible solution $\pi$ for this problem. Then note that when we increase or decrease all of its elements with the same number, these changes cancel out in the objective as well as in the constraints. Therefore it is possible to add the constraint $\pi_{s}=0$, preserving at least one of the optimal solutions. Furthermore, note that for optimal solutions $f^{*}$ and $\pi^{*}$ we have by complementary slackness that arcs with a positive flow, i.e. $f_{i j}^{*}>0$, imply that $\pi_{j}^{*}-\pi_{i}^{*}=\ell_{i j}$. Together with $\pi_{s}^{*}=0$ this implies that $\pi_{i}^{*}$ equals the shortest path length from $s$ to $i$.

\subsubsection{Parametric shortest path tree problem}

Now we assume that the length of the arcs is not known in advance but given by a linear function of variables. For each arc $(i, j)$ we have constant $c^{i j} \in \mathbb{R} \backslash\{0\}$, and for each node $j$ we have a variable $x^{j} \in[0,1]$. The length of arc $(i, j)$ is described by the product of $c^{i j}$ and $x^{j}$, i.e. $\ell_{i j}=c^{i j} x^{j}$.

The goal of the parametric shortest path tree problem (PSPT) is to choose the variables $x^{j} \in[0,1]$ in such a way that the sum of all (directed) shortest path lengths originating in the source node $s$ is maximized. Note that the problem is trivial if all constants are positive, as then it is optimal to choose all parameter-variables $\left(x^{j}\right)$ equal to the upper bound. Similar, when all constants are negative it is optimal to choose all parameters equal to zero. Let $x=\left(x^{i}\right)_{i \in V}$ and define $P_{i}(x)$ as the length of the shortest path from $s$ to node $i$ given $x$. The optimization problem is then:

$$
\max _{x} \sum_{i \in V \backslash\{s\}} P_{i}(x)
$$


subject to:

(PSPT)

$$
\begin{aligned}
& x^{i} \leq 1, \quad \forall i \in V \\
& x^{i} \geq 0, \quad \forall i \in V .
\end{aligned}
$$

We may observe that the definition of $P_{i}(x)$ implies itself an optimization problem, namely, problem (SPT) with $\ell_{i j}=c^{i j} x^{j}$ for all $\operatorname{arcs}(i, j) \in A$.

As both (Dual-SPT) and (PSPT) are maximizing the sum of the shortest path lengths, we will directly write the intersection of the two feasible regions and use one objective function, resulting in a linear programming formulation for the parametric shortest path tree problem:

$$
\max _{x, \pi} \sum_{i \in V \backslash\{s\}} \pi_{i}
$$

subject to:

$$
\begin{aligned}
\pi_{j}-\pi_{i} & \leq c^{i j} x^{j}, \quad \forall(i, j) \in A, \\
\pi_{s} & =0 \\
x^{i} & \leq 1, \quad \forall i \in V, \\
x^{i} & \geq 0, \quad \forall i \in V .
\end{aligned}
$$

Note that (LP) is a linear program, and is therefore polynomially solvable, see Papadimitriou [38]. Let $\left(x^{*}, \pi^{*}\right)$ be an optimal solution of this problem. Then $\pi_{i}^{*}$ is equal to the shortest path from $s$ to $i$ when $x=x^{*}$, i.e. $\pi_{i}^{*}=P_{i}\left(x^{*}\right)$.

\subsection{Optimality condition}

In this section we give a necessary and sufficient condition for an optimal solution of (LP). The condition has the nice property that it says that all nodes should fulfill a local optimality condition.

We start with the assumption that $x$ is such that no negative-length cycles exist, and consequently a shortest path is well-defined. For $x^{j}=0, \forall j \in V$, this holds trivially. Furthermore, we only consider other values of $x$ that induce longer shortest path lengths than the trivial solution. Let $\pi_{i}(x)$ denote the shortest path length from $s$ to $i$ given the parameter values $x$. Furthermore let $z_{i j}(x)$ be such that $\pi_{j}(x)-\pi_{i}(x)+z_{i j}(x)=c^{i j} x^{j}$, i.e. $z_{i j}(x)$ is the slack value in constraint (6.1). For given $x$, an arc $(i, j)$ is tight if $z_{i j}(x)=0$, which means that the shortest path length to $j$ is as long as the shortest path length to $i$ plus the arc length of $(i, j)$. As a result we have that tight arc $(i, j)$ is on a shortest 
path to $j$, where this path is not necessarily a simple path, i.e. one without loops. For a feasible solution $(x, \pi(x))$ being an optimal solution for (LP) the following condition should hold for all nodes. (Recall that $c^{i j}$ is nonzero for all $\operatorname{arcs}(i, j) \in A$.)

Condition 6.3.1 For node $j \in V$ exactly one of the following possibilities holds:

- $x^{j}=0$ and $\exists i$ such that both $c^{i j}<0$ and $(i, j)$ is tight.

- $x^{j}=1$ and $\exists i$ such that both $c^{i j}>0$ and $(i, j)$ is tight.

- $0<x^{j}<1$ and $\left\{\begin{array}{l}\exists i \text { such that both } c^{i j}<0 \text { and }(i, j) \text { is tight, and } \\ \exists i \text { such that both } c^{i j}>0 \text { and }(i, j) \text { is tight. }\end{array}\right.$

It is easy to see that if Condition 6.3.1 does not hold for some node $j$, the solution can be improved by changing $x^{j}$ marginally in an appropriate direction. In the following we prove that satisfying Condition 6.3.1 for all nodes $j \in V$ is also sufficient for optimality. We first need the following definitions and observation. Define $A(x)$ as the set of tight arcs corresponding to $x$, and $f(x)$ the total length of the shortest paths when the arc lengths are fixed by $x$. Note that we assume that there exists a path from $s$ to any other node, so there exists a shortest path tree $T \subseteq A(x)$.

Observation 6.3.2 Directed cycles of tight arcs have zero length.

\section{Proof:}

Consider cycle $C=\left(i_{1}, i_{2}\right),\left(i_{2}, i_{3}\right), \ldots,\left(i_{k}, i_{1}\right)$ of tight arcs. For each tight arc $(i, j)$ we know that $\pi_{j}(x)-\pi_{i}(x)=c^{i j} x^{j}$. If we add these equalities of all cycle arcs, we get $0=\sum_{(i, j) \in C} c^{i j} x^{j}$.

Now we prove that satisfying Condition 6.3.1 for all nodes $j \in V$ is also sufficient for optimality.

Theorem 6.3.3 A feasible solution $(x, \pi(x))$ is an optimal solution for (LP) if it satisfies Condition 6.3.1 for any node $j \in V$.

\section{Proof:}

The proof is by contradiction. Suppose we have a feasible solution $(\tilde{x}, \pi(\tilde{x}))$ for which the condition holds but which is not optimal. Let $(\dot{x}, \pi(\dot{x}))$ be an optimal solution, so $f(\dot{x})>f(\tilde{x})$. Let $T$ be a shortest path tree for solution $\tilde{x}$, i.e. $T \subseteq A(\tilde{x})$.

We now consider what happens to the arc and path lengths in $T$ when we step from $\tilde{x}$ to $\dot{x}$. As $f(\dot{x})>f(\tilde{x})$ there is at least one path in $T$ from $s$ to another node $t$ that 
gets longer. In the following we will trace back a path of tight arcs to $t$ that contains a cycle. We will see that this path and the cycle only contain arcs that get shorter or arcs that do not change in length when we step from $\tilde{x}$ to $\dot{x}$. Moreover, at least one of them strictly gets shorter. According to Observation 6.3.2 the cycle becomes a negative length cycle, contradicting the optimality assumption. Starting in $j_{0} \equiv t$ we trace back a path as follows, where $[i, j] \subseteq T$ denotes the path between $i$ and $j$ (possibly with $i=j$ ):

- Start in $j_{0}$, and trace back in $T$ until we find an arc that gets longer, which we denote by $\left(o_{1}, i_{1}\right)$. Note that as the path $\left[i_{1}, j_{0}\right]$ does not get longer, necessarily the path to $i_{1}$ gets longer.

- We know that the change of $\tilde{x}^{i_{1}}$ in $\dot{x}^{i_{1}}$ :

- increases the length of $\left(o_{1}, i_{1}\right) \in A(\tilde{x})$, as assumed by the choice of $i_{1}$, and

- decreases the length of some tight arc $\left(j_{1}, i_{1}\right) \in A(\tilde{x})$, as Condition 6.3.1 holds for $\tilde{x}$.

- This means that the path to $j_{1}$ necessarily gets longer, as otherwise the path via $j_{1}$ to $i_{1}$ shortens the path to $i_{1}$ and $j_{0}$.

- We can apply the same argument on $j_{1}$ as we did for $j_{0}$.

- We repeat this reasoning until the path $\ldots,\left(j_{2}, i_{2}\right),\left[i_{2}, j_{1}\right],\left(j_{1}, i_{1}\right),\left[i_{1}, j_{0}\right]$ forms a cycle, which will happen as there are finitely many nodes.

So we found a cycle containing (at least two) arcs $\left(j_{k}, i_{k}\right)$ whose lengths are reduced and paths $\left[i_{k+1}, j_{k}\right]$ whose lengths either decrease or remain the same. So, the length of the cycle is strictly smaller for $\dot{x}$ than for $\tilde{x}$. According to Observation 6.3.2 this means that we have a negative-length cycle, contradicting optimality of $\dot{x}$.

To illustrate the reasoning of Theorem 6.3.3 we have the following example.

Example 6.3.4 In Figure 6.1 we present an example. The figure shows a part of the shortest path tree (solid arcs) plus some arcs that we find by tracing back the path (dotted arcs). Note that all these arcs are in $A(\tilde{x})$. All arcs in the path are in a bold style. Furthermore, we indicated whether the arc gets longer, shorter or does not change in length by,+- and 0 . Starting with the observation that the path to $j_{0}=t$ gets longer, we start in $t$ and go back in the tree until we have an arc that gets longer $\left(o_{1}, i_{1}\right)$. As the condition holds, we then find a tight arc coming from another branch of the tree that gets shorter, $\left(j_{1}, i_{1}\right)$. As 
this arc was tight and gets shorter, we know that the path to $j_{1}$ gets longer. From $j_{1}$ we trace back in the tree until we find the first arc that gets longer, $\left(o_{2}, i_{2}\right)$, where $o_{2}=s$. Again we can find a tight arc coming from another branch of the tree that gets shorter, $\left(j_{2}, i_{2}\right)$. From $\left(j_{2}, i_{2}\right)$ on we can apply the same reasoning as we did for $\left(j_{1}, i_{1}\right)$, which gives an $\operatorname{arc}\left(j_{3}, i_{3}\right)$, and so on.

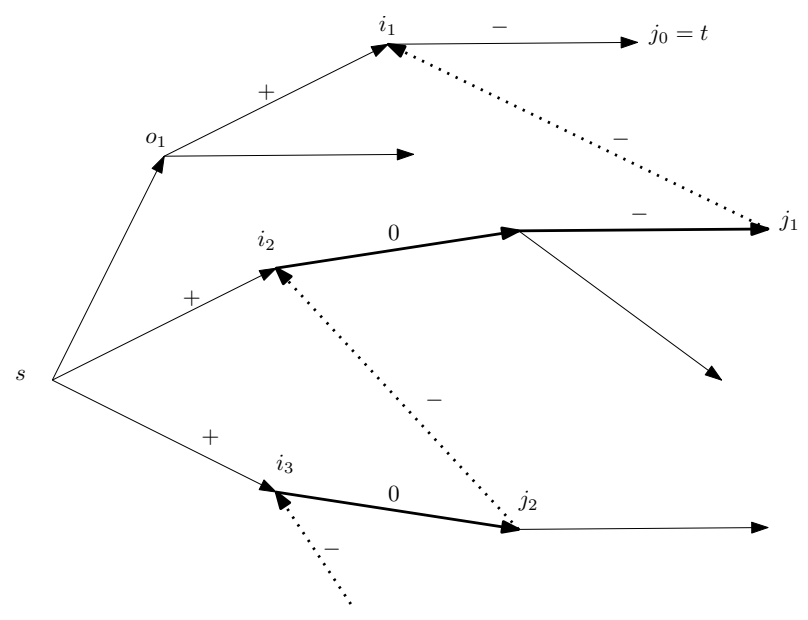

Figure 6.1: Path in $A(\tilde{x})$

\subsection{Local improvement algorithm}

\subsubsection{Algorithm and example}

In this section we present an algorithm for the parametric shortest path tree problem. Condition 6.3.1 suggests a straightforward local improvement algorithm. The main idea is to increase the shortest path lengths by changing one parameter at a time. We describe this algorithm and show that, unfortunately, it may need an infinite number of steps to finish.

Recall that $c^{i j}$ is nonzero for all arcs $(i, j) \in A$, and let $I_{j}(x)=\left\{\left(i_{1}, j\right), \ldots,\left(i_{r}, j\right)\right\}$ be the set of tight incoming arcs of node $j \in V$. For each node $j \in V$ we distinguish three cases:

Case $1 x^{j}<1$ and $c^{i j}>0 \quad \forall(i, j) \in I_{j}(x)$,

Case $2 x^{j}>0$ and $c^{i j}<0 \quad \forall(i, j) \in I_{j}(x)$,

Case 3 Condition 6.3.1 holds. 
One can see that Case 1 and Case 2 give an immediate possibility to increase the shortest path length to node $j$. For Case 1 any small enough increase in $x^{j}$ will do, and for Case 2 any small enough decrease in $x^{j}$ will do. Now the question is how and how much to increase or decrease $x^{j}$ in these two cases.

Let $V_{j}(x)$ denote the set of nodes for which every shortest path passes $j$, with $j \notin$ $V_{j}(x)$. So, in the shortest arc network, all nodes in $V_{j}(x)$ are linked to $s$ exclusively via $j$. Let node $j$ be of Case 1 or Case 2, and consider what happens if we gradually change $x^{j}$ within its lower and upper bound. As long as no new arc gets tight and we do not reach the bounds, we may continue changing $x^{j}$, which will increase $\pi_{j}$ and $\pi_{i}$ for all $i \in V_{j}(x)$ with the same number. Note that $\pi_{i}, i \in V_{j}(x)$, increases as all its shortest paths pass node $j$.

Formally, let $\Delta_{j}$ denote the change in $x^{j}$, positive for Case 1 and negative for Case 2. The shortest path length to $j$ and the nodes in $V_{j}(x)$ increases by $c^{i * j} \Delta_{j}$, with $i^{*}$ such that $\left|c^{i * j}\right| \leq\left|c^{i j}\right|, \quad \forall(i, j) \in I_{j}(x)$. There are four factors that bound the value of $\Delta_{j}$ :

- First, a further change in $x^{j}$ would cause infeasibility, i.e. $x^{j}$ reaches its bounds: $x^{j}+\Delta_{j} \in\{0,1\}$.

- Second, an arc $(i, j)$ with $i \in V_{j}(x)$ becomes tight which means that a further change in $x^{j}$ will create a negative cycle containing arc $(i, j)$.

- Third, an arc $(i, j)$ with $i \notin V_{j}(x)$ becomes tight which means that a further change in $x^{j}$ will shorten the path to $j$.

- Fourth, an alternative shortest path arises to a node in $V_{j}(x)$, which means that this set needs to be updated.

The first three factors also end the current possibility to improve on node $j$.

According to the first factor, $x^{j}$ becomes infeasible if it is increased by more than $\alpha_{1} \equiv 1-x^{j}$ or if it is decreased by more than $\alpha_{2} \equiv x^{j}$.

For the second factor, consider $\pi_{j}(x)-\pi_{i}(x) \leq c^{i j} x^{j}$, with $i \in V_{j}(x)$, where the left hand side remains constant as both the paths to $i$ and $j$ change with the same amount. The constraint gets tight when the right hand side is reduced with $z_{i j}(x)$, the original slack. Therefore, arc $(i, j)$ will only get tight if $c^{i j}$ and the change in $x^{j}$ have opposite signs. For Case 1 this gives a maximum increase in $x^{j}$ of

$$
\beta_{1} \equiv \min \left\{u \mid u=-z_{i j}(x) / c^{i j}, \quad i \in V_{j}(x), \quad(i, j) \in A, \quad c^{i j}<0\right\},
$$


and for Case 2 we have a maximum decrease in $x^{j}$ of

$$
\beta_{2} \equiv \min \left\{u \mid u=z_{i j}(x) / c^{i j}, \quad i \in V_{j}(x), \quad(i, j) \in A, \quad c^{i j}>0\right\} .
$$

For the third factor, consider $\pi_{j}(x)-\pi_{i}(x) \leq c^{i j} x^{j}$, with $i \notin V_{j}(x)$. When we change $x$, this also changes $\pi_{j}(x)$ and $c^{i j} x^{j}$, while $\pi_{i}(x)$ does not change. The arc can get tight if $c^{i j} x^{j}$ decreases, or increases less than $\pi_{j}(x)$. For Case 1 this gives a maximum increase in $x^{j}$ of

$$
\gamma_{1} \equiv \min \left\{u \mid u=\frac{z_{i j}(x)}{c^{i^{*} j}-c^{i j}}, \quad i \notin V_{j}(x), \quad(i, j) \in A, \quad c^{i j}<c^{i^{*} j}\right\}
$$

and for Case 2 we have a maximum decrease in $x^{j}$ of

$$
\gamma_{2} \equiv \min \left\{u \mid u=-\frac{z_{i j}(x)}{c^{i^{*} j}-c^{i j}}, \quad i \notin V_{j}(x), \quad(i, j) \in A, \quad c^{i j}>c^{i^{*} j}\right\} .
$$

Finally, the fourth factor is that we stop changing $x$ when $V_{j}(x)$ needs to be updated. This happens if there arises a shortest path to one of its nodes that does not pass node $j$, i.e. if an arc $(k, i)$ with $k \notin V_{j}(x)$ and $i \in V_{j}(x)$ becomes tight. Consider $\pi_{i}(x)-\pi_{k}(x) \leq$ $c^{k i} x^{i}$ where $\pi_{i}(x)$ is the only part that changes. So the arcs gets tight when the increase in $\pi_{i}$ is equal to the original slack $z_{k i}(x)$. This gives the following maximum change in $x^{j}:$

$$
\delta \equiv \min \left\{u\left|u=z_{k i}(x) /\right| c^{i^{*} j} \mid, \quad k \notin V_{j}(x), \quad i \in V_{j}(x), \quad(k, i) \in A\right\} .
$$

Concluding, in Case 1 we increase $x^{j}$ by $\min \left\{\alpha_{1}, \beta_{1}, \gamma_{1}, \delta\right\}$, in Case 2 we decrease $x^{j}$ by $\min \left\{\alpha_{2}, \beta_{2}, \gamma_{2}, \delta\right\}$, and in both cases we increase $\pi_{i}$ by $\left|c^{i^{*} j} \Delta_{j}\right|$ for all nodes $i$ in $V_{j}(x)$ and node $j$, where $\Delta_{j}$ is the change in $x^{j}$. We now have the following algorithm.

\section{Local improvement algorithm}

Step 0. Initialization: start with $x^{j}=0$, and therefore $\pi_{j}=0$ for all $j \in V$. So all arcs are tight.

Step 1. Determine $I_{i}(x)$ for all $i \in V$. Choose a node $j \in V$ for which Case 1 or Case 2 holds. If no such node exists, go to step 5 .

Step 2. Determine respectively $V_{j}(x), i^{*}, \alpha_{1}$ or $\alpha_{2}, \beta_{1}$ or $\beta_{2}, \gamma_{1}$ or $\gamma_{2}$, and $\delta$.

If $j$ of Case 1 , let $\Delta^{j}=\min \left\{\alpha_{1}, \beta_{1}, \gamma_{1}, \delta\right\}$.

If $j$ of Case 2 , let $\Delta^{j}=-\min \left\{\alpha_{2}, \beta_{2}, \gamma_{2}, \delta\right\}$.

Step 3. Set $x^{j}:=x^{j}+\Delta_{j}$.

Set $\pi_{j}:=\left|c^{i^{*} j} \Delta_{j}\right|$.

Set $\pi_{i}:=\pi_{i}+\left|c^{i^{*} j} \Delta_{j}\right|$ for all $i \in V_{j}(x)$. 
Step 4. If $j$ of Case 1 and $\Delta_{j}<\min \left\{\alpha_{1}, \beta_{1}, \gamma_{1}\right\}$, then go to Step 2 .

If $j$ of Case 2 and $\Delta_{j}<\min \left\{\alpha_{2}, \beta_{2}, \gamma_{2}\right\}$, then go to Step 2 . Go to Step 1.

\section{Step 5. Stop.}

Obviously, if the local improvement algorithm terminates, we have a solution fulfilling Condition 6.3.1 for all nodes $j \in V$, and hence we have an optimal solution according to Theorem 6.3.3. However, the algorithm may need an infinite number of steps, as will be shown in the following example.

Example 6.4.1 Consider a graph with source node $s$ and three other nodes, 1, 2 and 3. The arcs and the values of $c^{i j}$ are depicted in Figure 6.2. First observe that the solution

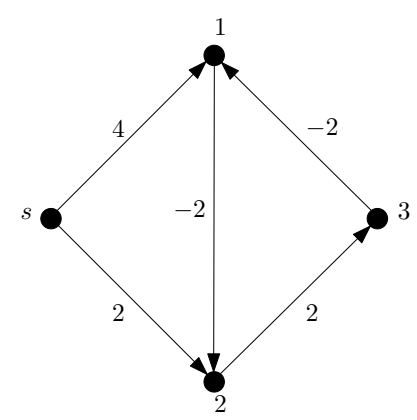

Figure 6.2: Graph $G$ and values of $c^{i j}$

$x=\left(\frac{1}{2}, \frac{1}{2}, 1\right), \pi=(2,1,3)$ fulfills Condition 6.3.1 and is therefore optimal according to Theorem 6.3.3. We apply the local improvement algorithm on this example.

Step 0. $x^{1}=x^{2}=x^{3}=0$, and therefore $\pi_{1}=\pi_{2}=\pi_{3}=0$. So all arcs are tight.

Step 1. $I_{1}(x)=\{(s, 1),(3,1)\}, I_{2}(x)=\{(s, 2),(1,2)\}, I_{3}(x)=\{(2,3)\}$. The only node that does not fulfill Condition 6.3.1 is node 3.

Step 2. $V_{3}(x)=\varnothing, i^{*}=2, \alpha_{1}=1, \beta_{1}=\infty, \gamma=\infty$, and $\delta=\infty$. As 3 is of Case 1, let $\Delta_{3}=\min \left\{\alpha_{1}, \beta_{1}, \gamma_{1}, \delta\right\}=1$.

Step 3. $x^{3}=0+1$. $\pi_{3}=0+|2 \times 1|=2$.

Step 4. As $x^{3}=1$ we go to step 1 .

Step 1. $I_{1}(x)=\{(s, 1)\}, I_{2}(x)=\{(s, 2),(1,2)\}, I_{3}(x)=\{(2,3)\}$. The only node that does not fulfill Condition 6.3.1 is node 1. 
Step 2. $V_{1}(x)=\varnothing, i^{*}=s, \alpha_{1}=1, \beta_{1}=\infty, \gamma_{1}=\frac{2}{4-(-2)}=\frac{1}{3}$, and $\delta=\infty$. As 1 is of Case 1, let $\Delta_{1}=\min \left\{\alpha_{1}, \beta_{1}, \gamma_{1}, \delta\right\}=\frac{1}{3}$.

Step 3. $x^{1}=0+\frac{1}{3}$. $\pi_{1}=0+\left|4 \times \frac{1}{3}\right|=\frac{4}{3}$.

Step 4. As $\Delta_{1}=\gamma_{1}$ we go to step 1 .

Step 1. $I_{1}(x)=\{(s, 1),(3,1)\}, I_{2}(x)=\{(s, 2)\}, I_{3}(x)=\{(2,3)\}$. The only node that does not fulfill Condition 6.3.1 is node 2.

Step 2. $V_{2}(x)=\{3\}, i^{*}=1, \alpha_{1}=1, \beta_{1}=\infty, \gamma=\frac{\frac{4}{3}}{2-(-2)}=\frac{1}{3}$, and $\delta=\infty$. As 2 is of Case 1, let $\Delta_{2}=\min \left\{\alpha_{1}, \beta_{1}, \gamma_{1}, \delta\right\}=\frac{1}{3}$.

Step 3. $x^{2}=0+\frac{1}{3}$.

$$
\begin{aligned}
& \pi_{2}=0+\left|2 \times \frac{1}{3}\right|=\frac{2}{3} . \\
& \pi_{3}=2+\left|2 \times \frac{1}{3}\right|=2 \frac{2}{3} .
\end{aligned}
$$

Step 4. As $\Delta_{2}=\gamma_{1}$ we go to step 1 .

The algorithm continues by alternately applying the steps 1 to 4 on node 1 and 2 . After the last step arc $(1,2)$ became tight, while $(3,1)$ lost its tightness. This means that $x^{1}$ can be increased until $(3,1)$ is tight again, but then $(1,2)$ loses tightness, giving the opportunity to increase $x^{2}$, etcetera. One can see that $x^{1}<\frac{1}{2}$ if $x^{2}<\frac{1}{2}$, and $x^{2}<\frac{1}{2}$ if $x^{1}<\frac{1}{2}$. One increases $x^{1}$ until the path $(s, 1)$ has the same length as the path $(s, 2),(2,3),(3,1)$, i.e. until $4 x^{1}=2 x^{2}+2-2 x^{1}$, so $x^{1}=\frac{1}{3} x^{2}+\frac{1}{3}$. One increases $x^{2}$ until the path $(s, 2)$ has the same length as the path $(s, 1),(1,2)$, i.e. until $2 x^{2}=4 x^{1}-2 x^{2}$, so $x^{2}=x^{1}$. As we start in a solution where both $x^{1}$ and $x^{2}$ are smaller than a half, we will never reach the optimal solution in a finite number of steps.

\subsubsection{Accelerating the algorithm}

The algorithm may get caught in an infinitely repeated loop, as is shown in example 6.4.1. In this section we show that we can handle these loops efficiently by repeating the first loop in one step.

Let $\left(x_{A}, \pi^{A}\right)$ and $\left(x_{B}, \pi^{B}\right)$ be two feasible solutions for (LP), and assume that $\pi^{A}=$

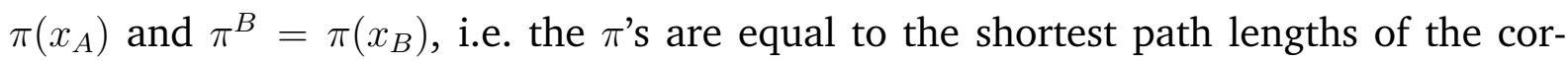
responding solution for $x$. Assume that $\sum_{i \in V} \pi_{i}^{A}<\sum_{i \in V} \pi_{i}^{B}$. Furthermore, define $\delta x \equiv x_{B}-x_{A}$ and $\delta \pi \equiv \pi^{B}-\pi^{A}$. Consider the following solution:

$$
\left(x_{C}, \pi^{C}\right) \equiv\left(x_{B}, \pi^{B}\right)+F \cdot(\delta x, \delta \pi)
$$


in which $F$ is the largest real number that makes $\left(x_{C}, \pi^{C}\right)$ a feasible solution of (LP). Note that $F$ is nonnegative as $F=0$ is a trivial solution. Then we have the following observation for solution $\left(x_{C}, \pi^{C}\right)$.

Theorem 6.4.2 Arcs that are tight in solution $\left(x_{B}, \pi^{B}\right)$, are also tight in solution $\left(x_{C}, \pi^{C}\right)$.

\section{Proof:}

The theorem is trivial for $F=0$, so assume $F>0$. The proof is by contradiction. Suppose $\operatorname{arc}(i, j)$ is tight for solution $\left(x_{B}, \pi^{B}\right)$, and not tight for solution $\left(x_{C}, \pi^{C}\right)$. Consider the corresponding constraint for $A, B$ and $C$ :

$$
\begin{aligned}
\pi_{j}^{A}-\pi_{i}^{A} \leq c^{i j} x_{A}^{j}, \\
\pi_{j}^{B}-\pi_{i}^{B}=c^{i j} x_{B}^{j}, \\
\pi_{j}^{C}-\pi_{i}^{C}<c^{i j} x_{C}^{j} .
\end{aligned}
$$

Using the definitions of $\left(x_{C}, \pi^{C}\right)$ and $(\delta x, \delta \pi)$ we can write

$$
\left(x_{B}, \pi^{B}\right)=\frac{F}{1+F}\left(x_{A}, \pi^{A}\right)+\frac{1}{1+F}\left(x_{C}, \pi^{C}\right) .
$$

Implying that (6.4) is a convex combination of (6.3) and (6.5). This contradicts the signs of the constraint for the three different solutions.

We can apply this result to extend the local improvement algorithm. Suppose that $\left(v_{1}, \ldots, v_{a}, \ldots, v_{b}\right)$ is the sequence of nodes for which the variable $x$ is changed during the algorithm and let $v_{a}=v_{b}$, i.e. the sequence is looping. Instead of continuing local improvements we can now first eliminate the possibility of infinite repetition of this loop by applying (6.2) with $\left(x_{A}, \pi^{A}\right)$ the solution before updating node $v_{a}$ and $\left(x_{B}, \pi^{B}\right)$ the solution before (considering) updating node $v_{b}$. After this step the local improvement algorithm can be continued again as we have that $\pi^{C}=\pi\left(x_{C}\right)$ if this is also true for $\pi^{A}=\pi\left(x_{A}\right)$ and $\pi^{B}=\pi\left(x_{B}\right)$ :

Proposition 6.4.3 If $\pi^{A}=\pi\left(x_{A}\right)$ and $\pi^{B}=\pi\left(x_{B}\right)$ then also $\pi^{C}=\pi\left(x_{C}\right)$, i.e. $\pi^{C}$ equals the shortest path lengths given $x_{C}$.

\section{Proof:}

We know from Theorem 6.4.2 that all tight arcs (and therefore any shortest path tree) from solution $\left(x_{B}, \pi^{B}\right)$ are also tight for $\left(x_{C}, \pi^{C}\right)$ which implies that $\pi^{C}=\pi\left(x_{C}\right)$. 
Unfortunately, even by using this kind of loop elimination, finiteness is still not guaranteed. This can be seen by extending the graph of Example 6.4.1, by adding its mirror image, see picture 6.3. The upper part of the graph is the original graph, where it has arc $(s, 2)$ in common with its mirror image. In this graph, the algorithm can get stuck

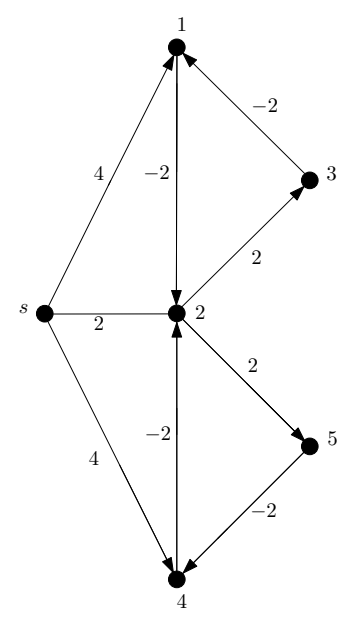

Figure 6.3: Graph $G$ and values of $c^{i j}$

in a loop of loops: The loop between nodes 1 and 2 can be handled until arc $(s, 4)$ gets tight, whereafter the loop between nodes 4 and 2 can be handled until arc $(s, 1)$ gets tight, and so on. Like Example 6.4.1, $x^{2}$ can only have its optimal value if $x^{1}$ and $x^{4}$ are equal to their optimal values, and the other way around. So, to be able to apply the algorithm acceleration effectively, it should be applied on the largest loop. However, this can only be done if you know in advance what the largest loop is.

\subsection{Alternative formulation}

In this section we present an alternative formulation for the parametric shortest path tree problem (LP). Using the structure of an optimal solution, we are able to eliminate the parametric variables.

\subsubsection{Elimination of parametric variables}

Consider an optimal solution of (LP). Then we know from Theorem 6.3.3 and Condition 6.3.1 that for node $k$ we have at least one or two tight arcs. Knowing the tight arcs of node $k$, we are able to express $x^{k}$ in terms of $\pi$. Recall that $c^{i j}$ is nonzero for all arcs $(i, j) \in A$. For the three possibilities we have respectively: 
- Let $(j, k)$ be the tight arc with $c^{j k}<0$. By definition $x^{k}=0$, which gives $\pi_{k}=\pi_{j}$.

- Let $(i, k)$ be the tight arc with $c^{i k}>0$. By definition $x^{k}=1$, which gives $\pi_{k}=$ $\pi_{i}+c^{i k}$.

- Let $(i, k)$ and $(j, k)$ be tight arcs with $c^{i k}>0$ and $c^{j k}<0$. Then we may solve the two corresponding constraints, giving $x^{k}=\frac{\pi_{j}-\pi_{i}}{c^{i k}-c^{j k}}$. Substituting in either of the constraints gives $\pi_{k}$ as a convex combination of $\pi_{i}$ and $\pi_{j}$ :

$$
\pi_{k}=\frac{c^{i k}}{c^{i k}-c^{j k}} \pi_{j}+\frac{-c^{j k}}{c^{i k}-c^{j k}} \pi_{i} .
$$

Proposition 6.5.1 The following inequalities hold for any feasible solution:

- For any $\operatorname{arc}(j, k)$ with $c^{j k}<0$ we have $\pi_{k}-\pi_{j} \leq c^{j k} x^{k} \leq 0$, which means $\pi_{k} \leq \pi_{j}$.

- For any arc $(i, k)$ with $c^{i k}>0$ we have $\pi_{k}-\pi_{i} \leq c^{i k} x^{k} \leq c^{i k}$, which means $\pi_{k} \leq$ $\pi_{i}+c^{i k}$.

- For any combination of arcs $(i, k)$ and $(j, k)$ with $c^{i k}>0$ and $c^{j k}<0$ we can eliminate $x^{k}$ from the system of inequalities $\pi_{k}-\pi_{j} \leq c^{j k} x^{k}$ and $\pi_{k}-\pi_{i} \leq c^{i k} x^{k}$ which gives

$$
\pi_{k} \leq \frac{c^{i k}}{c^{i k}-c^{j k}} \pi_{j}+\frac{-c^{j k}}{c^{i k}-c^{j k}} \pi_{i} .
$$

Let $A^{+}$be the set of all arcs $(i, j)$ with $c^{i j}>0$, and $A^{-} \equiv A \backslash A^{+}$. Consider the following linear program:

$$
\max _{\pi} \sum_{i \in V \backslash\{s\}} \pi_{i}
$$

subject to:

$$
\begin{aligned}
\pi_{k} & \leq \pi_{j}, \quad \forall(j, k) \in A^{-}, \\
\pi_{k} & \leq \pi_{i}+c^{i k}, \quad \forall(i, k) \in A^{+}, \\
\pi_{k} & \leq \frac{c^{i k}}{c^{i k}-c^{j k}} \pi_{j}+\frac{-c^{j k}}{c^{i k}-c^{j k}} \pi_{i}, \quad \forall((i, k),(j, k)) \in A^{+} \times A^{-}, \\
\pi_{s} & =0 .
\end{aligned}
$$

As all constraints in (AF) follow from (LP), we know that the optimal $\pi$ from (LP) is also feasible in (AF). The reverse is also true.

Proposition 6.5.2 An optimal solution of (AF) corresponds to a feasible solution of (LP). 


\section{Proof:}

Let $\pi$ be an optimal solution for (AF). We show that we can choose $x$ such that $(\pi, x)$ is a feasible solution in (LP). Consider node $k$ and all its upper bounds in (AF). By optimality in (AF), at least one of these upper bounds is tight. First, suppose there exists a pair of arcs $((i, k),(j, k)) \in A^{+} \times A^{-}$such that $\pi_{k}=\frac{c^{i k}}{c^{i k}-c^{j k}} \pi_{j}+\frac{-c^{j k}}{c^{i k}-c^{j k}} \pi_{i}$. Then $x^{k}=\frac{\pi_{j}-\pi_{i}}{c^{i k}-c^{j k}}$ makes that $\pi_{k}-\pi_{\ell} \leq c^{\ell k} x^{k}$ holds for all $(\ell, k) \in A$. This can be seen as follows. By feasibility in (AF) we have for $c^{\ell k}>0$ that

$$
\frac{c^{i k}}{c^{i k}-c^{j k}} \pi_{j}+\frac{-c^{j k}}{c^{i k}-c^{j k}} \pi_{i} \leq \frac{c^{\ell k}}{c^{\ell k}-c^{j k}} \pi_{j}+\frac{-c^{j k}}{c^{\ell k}-c^{j k}} \pi_{\ell},
$$

where subtracting $\pi_{j}$ at both sides gives

$$
c^{j k} \frac{\pi_{j}-\pi_{i}}{c^{i k}-c^{j k}} \leq c^{j k} \frac{\pi_{j}-\pi_{\ell}}{c^{\ell k}-c^{j k}},
$$

showing $\frac{\pi_{j}-\pi_{i}}{c^{i k}-c^{j k}} \geq \frac{\pi_{j}-\pi_{\ell}}{c^{\ell k}-c^{j k}}$. Using this we have by feasibility in (AF)

$$
\pi_{k} \leq \frac{c^{\ell k}}{c^{\ell k}-c^{j k}} \pi_{j}+\frac{-c^{j k}}{c^{\ell k}-c^{j k}} \pi_{\ell}=\pi_{\ell}+c^{\ell k}\left(\frac{\pi_{j}-\pi_{\ell}}{c^{\ell k}-c^{j k}}\right) \leq \pi_{\ell}+c^{\ell k} x^{k} .
$$

Similarly for $c^{\ell k}<0$ we have

$$
\frac{c^{i k}}{c^{i k}-c^{j k}} \pi_{j}+\frac{-c^{j k}}{c^{i k}-c^{j k}} \pi_{i} \leq \frac{c^{i k}}{c^{i k}-c^{\ell k}} \pi_{\ell}+\frac{-c^{\ell k}}{c^{i k}-c^{\ell k}} \pi_{i}
$$

where subtracting $\pi_{i}$ at both sides gives

$$
c^{i k} \frac{\pi_{j}-\pi_{i}}{c^{i k}-c^{j k}} \leq c^{i k} \frac{\pi_{\ell}-\pi_{i}}{c^{i k}-c^{\ell k}}
$$

showing $\frac{\pi_{j}-\pi_{i}}{c^{i k}-c^{j k}} \leq \frac{\pi_{\ell}-\pi_{i}}{c^{i k}-c^{\ell k}}$. Using this we have by feasibility in (AF)

$$
\pi_{k} \leq \frac{c^{i k}}{c^{i k}-c^{\ell k}} \pi_{\ell}+\frac{-c^{\ell k}}{c^{i k}-c^{\ell k}} \pi_{i}=\pi_{\ell}+c^{\ell k}\left(\frac{\pi_{\ell}-\pi_{i}}{c^{i k}-c^{\ell k}}\right) \leq \pi_{\ell}+c^{\ell k} x^{k} .
$$

Second, suppose that there is not such a pair of arcs, but there is an $\operatorname{arc}(j, k) \in A^{-}$ such that $\pi_{k}=\pi_{j}$. Then $x^{k}=0$ makes that $\pi_{k}-\pi_{\ell} \leq c^{\ell k} x^{k}$ holds for all $(\ell, k) \in A$. For $(\ell, k) \in A^{-}$it follows trivially from feasibility in (AF). For $(\ell, k) \in A^{+}$we have $\pi_{k} \leq \frac{c^{\ell k}}{c^{\ell k}-c^{j k}} \pi_{j}+\frac{-c^{j k}}{c^{\ell k}-c^{j k}} \pi_{\ell}$ where replacing $\pi_{j}$ by $\pi_{k}$ gives

$$
\begin{aligned}
& \pi_{k} \leq \frac{c^{\ell k}}{c^{\ell k}-c^{j k}} \pi_{k}+\frac{-c^{j k}}{c^{\ell k}-c^{j k}} \pi_{\ell} \\
\Leftrightarrow \quad & \frac{-c^{j k}}{c^{\ell k}-c^{j k}} \pi_{k} \leq \frac{-c^{j k}}{c^{\ell k}-c^{j k}} \pi_{\ell} \\
\Leftrightarrow \quad & \pi_{k} \leq \pi_{\ell} .
\end{aligned}
$$


Third, suppose the previous two cases do not hold, but there is an $\operatorname{arc}(i, k) \in A^{+}$such that $\pi_{k}=\pi_{i}+c^{i k}$. Then $x^{k}=1$ makes that $\pi_{k}-\pi_{\ell} \leq c^{\ell k} x^{k}$ holds for all $(\ell, k) \in A$. For $(\ell, k) \in A^{+}$it follows trivially from feasibility in (AF). For $(\ell, k) \in A^{-}$we have $\pi_{k} \leq \frac{c^{i k}}{c^{i k}-c^{\ell k}} \pi_{\ell}+\frac{-c^{\ell k}}{c^{i k}-c^{\ell k}} \pi_{i}$ where replacing $\pi_{i}$ by $\pi_{k}-c^{i k}$ gives

$$
\begin{array}{ll} 
& \pi_{k} \leq \frac{c^{i k}}{c^{i k}-c^{\ell k}} \pi_{\ell}+\frac{-c^{\ell k}}{c^{i k}-c^{\ell k}}\left(\pi_{k}-c^{i k}\right) \\
\Leftrightarrow \quad & \frac{c^{i k}}{c^{i k}-c^{\ell k}} \pi_{k} \leq \frac{c^{i k}}{c^{i k}-c^{\ell k}} \pi_{\ell}+\frac{c^{i k}}{c^{i k}-c^{\ell k}} c^{\ell k} \\
\Leftrightarrow \quad & \pi_{k} \leq \pi_{\ell}+c^{\ell k} .
\end{array}
$$

We conclude that all constraints on $\pi$ in problem (LP) are fulfilled. The only thing still to be proven is that our choices for $x$ are such that $0 \leq x^{k} \leq 1$ for all $k \in V$. We use that $\pi_{k}=\frac{c^{i k}}{c^{i k}-c^{j k}} \pi_{j}+\frac{-c^{j k}}{c^{i k}-c^{j k}} \pi_{i}$ for some $((i, k),(j, k)) \in A^{+} \times A^{-}$if $x^{k} \notin\{0,1\}$. From $\pi_{k} \leq \pi_{j}$ we get

$$
\frac{c^{i k}}{c^{i k}-c^{j k}} \pi_{j}+\frac{-c^{j k}}{c^{i k}-c^{j k}} \pi_{i}=\pi_{j}+c^{j k}\left(\frac{\pi_{j}-\pi_{i}}{c^{i k}-c^{j k}}\right) \leq \pi_{j},
$$

which implies that $\frac{\pi_{j}-\pi_{i}}{c^{i k}-c^{j k}} \geq 0$ as $c^{j k}<0$. Finally, from $\pi_{k} \leq \pi_{i}+c^{i k}$ we get

$$
\frac{c^{i k}}{c^{i k}-c^{j k}} \pi_{j}+\frac{-c^{j k}}{c^{i k}-c^{j k}} \pi_{i}=\pi_{i}+c^{i k}\left(\frac{\pi_{j}-\pi_{i}}{c^{i k}-c^{j k}}\right) \leq \pi_{i}+c^{i k}
$$

which implies that $\frac{\pi_{j}-\pi_{i}}{c^{i k}-c^{j k}} \leq 1$ as $c^{i k}>0$.

Unfortunately, the new problem formulation does not solve the problem that a local improvement algorithm may be infinite.

Example 6.5.3 Consider Example 6.4.1. Reformulation of this problem gives (after elimination of $\pi_{s}$ and dominated restrictions):

$$
\max _{\pi} \sum_{i \in V} \pi_{i}
$$

subject to:

$$
\begin{aligned}
\pi_{3} & \leq \pi_{2}+2, \\
\pi_{1} & \leq \frac{2}{3} \pi_{3}, \\
\pi_{2} & \leq \frac{1}{2} \pi_{1} .
\end{aligned}
$$

Here it is easy to see that the optimal solution $(\pi=(2,1,3))$ can not be achieved by raising one element of $\pi$ at a time, starting from zero. From the first two constraints we get $\pi_{1} \leq \frac{2}{3} \pi_{2}+\frac{4}{3}$, implying that $\pi_{1}<2$ if $\pi_{2}<1$. However, the last constraint says that $\pi_{2}<1$ if $\pi_{1}<2$. 


\subsubsection{Open problem}

As (AF) is a linear program, we know it can be solved efficiently. However, as we know from the discussion above, there is no straightforward combinatorial algorithm that solves the problem efficiently. The question is, whether there exists an efficient algorithm that uses the combinatorial structure of the problem.

\subsection{Origin of parametric shortest path tree problems}

\subsubsection{Economic example: monopolist pricing}

Consider a monopolist of some (raw) material who maximizes its expected revenue. The preferences (called type) of a potential customer of the monopolist are denoted by $\theta$, and are unknown by the monopolist. The customer faces a quasi-linear utility function $u(q, \theta)=v(q, \theta)-p$, where $v(q, \theta)$ is its monetary valuation for having $q$ units of the material, and $p$ is the money transfer from the customer to the monopolist. Although the monopolist does not know the type $\theta$ of the customer, it is known that $\theta \in\left\{\theta_{1}, \ldots, \theta_{n}\right\}$, with probability $f_{j}$ being of type $\theta_{j}$.

The problem for the monopolist is to offer the customer a set of contracts $\left(q_{j}, p_{j}\right)$, $j=1, \ldots, n$, one for each possible type such that:

- A customer of type $\theta_{j}$ chooses contract $\left(q_{j}, p_{j}\right), j=1, \ldots, n$ (Incentive compatibility), and

- the chosen contract offers the customer a nonnegative utility (Rationality).

(The monopolist may choose to give different contracts the same value. The reason for having different values is to raise the expected profit.)

The set of contracts fulfills the incentive compatibility requirement if it holds that:

$$
v\left(q_{j}, \theta_{j}\right)-p_{j} \geq v\left(q_{i}, \theta_{j}\right)-p_{i}, \quad \forall i, j=1, \ldots, n,
$$

i.e. a customer of type $\theta_{j}$ maximizes its utility by choosing contract $\left(q_{j}, p_{j}\right)$. The set of contracts fulfills the rationality requirement if it also holds that:

$$
v\left(q_{j}, \theta_{j}\right)-p_{j} \geq 0, \quad \forall j=1, \ldots, n .
$$

Assume that any customer has a zero valuation for receiving no units, i.e. $v(0, \cdot)=$ 0 . By defining a dummy type $\theta_{0}$ and dummy contract $\left(q_{0}, p_{0}\right)=(0,0)$ the rationality constraints become part of the incentive compatibility constraints. 
The problem of the monopolist can be described by the following linear program:

$$
\max _{q, p} \sum_{i=1}^{n} f_{i} p_{i}
$$

subject to:

$(\mathrm{MON})$

$$
\begin{aligned}
p_{j}-p_{i} & \leq v\left(q_{j}, \theta_{j}\right)-v\left(q_{i}, \theta_{j}\right), \quad \forall i, j=0, \ldots, n, \\
p_{0} & =0, \\
q_{j} & \leq C, \quad \forall j=1, \ldots, n, \\
q_{j} & \geq 0, \quad \forall j=1, \ldots, n,
\end{aligned}
$$

where $C$ is the quantity available of the material. Notice that (MON) is (the dual formulation of) a parametric shortest path tree problem with (parametric) arc-length function $v\left(q_{j}, \theta_{j}\right)-v\left(q_{i}, \theta_{j}\right)$.

The problem of the monopolist is an example of what is called a screening game, see section 1.3.2. Note that the design of a mechanism is also an example of a screening game. However, the screening game of the monopolist is not a mechanism design problem as the rules of the game are exogenously given. The example above is based on the survey on screening of Rochet and Stole [42].

\subsubsection{Auction example: revenue maximization}

Consider an auction with $m$ bidders. The bidders are stochastically the same in the sense that the preferences (called type) of the bidders are random draws from the same discrete distribution. Let $\Lambda$ be the finite set of possible types, and $\lambda_{j}$ the type of bidder $j$, whose value is only known by bidder $j$. Let $\lambda \equiv\left(\lambda_{1}, \ldots, \lambda_{m}\right) \in \Lambda^{m}$. The probability that the $m$-tuple $\lambda$ is realized is denoted by $P(\lambda)$. We write $\left(\lambda_{j}, \lambda^{-j}\right)=\lambda$ to highlight the element from bidder $j$, and denote $P\left(\lambda^{-j}\right)$ the probability that $\lambda^{-j}$ is realized, ignoring the type of bidder $j$.

Bidder $j$ faces a quasi-linear utility function $u\left(\alpha, \lambda_{j}\right)=v\left(\alpha, \lambda_{j}\right)-p_{j}$, where $v(\alpha, \lambda)$ is its monetary valuation for allocation $\alpha$, and $p_{j}$ is the money transfer from bidder $j$ to the auctioneer. The auction is a direct revelation mechanism in which the bidders reveal their type, knowing that the allocation and payments are determined by an allocation function and payment function of the revealed types:

$$
\begin{aligned}
& \alpha: \quad \Lambda^{m} \rightarrow A, \\
& p: \quad \Lambda^{m} \rightarrow \mathbb{R}^{m},
\end{aligned}
$$

where $A$ is the set of possible allocations. These functions have to fulfill two conditions: 
- Incentive compatibility, i.e. reporting truthfully is a Bayes-Nash equilibrium, which means that reporting truthful is in expectation the best strategy, and

- Individual rationality, i.e. in expectation it is profitable for all bidders to participate in the auction.

The incentive compatibility constraints can be written as:

$$
\begin{aligned}
& \sum_{\lambda^{-j} \in \Lambda^{m-1}}\left[v\left(\alpha\left(\lambda_{j}, \lambda^{-j}\right), \lambda_{j}\right)-p_{j}\left(\lambda_{j}, \lambda^{-j}\right)\right] P\left(\lambda^{-j}\right) \\
& \geq \sum_{\lambda^{-j} \in \Lambda^{m-1}}\left[v\left(\alpha\left(\mu, \lambda^{-j}\right), \lambda_{j}\right)-p_{j}\left(\mu, \lambda^{-j}\right)\right] P\left(\lambda^{-j}\right), \\
& \forall j \in\{1, \ldots, m\}, \forall \lambda \in \Lambda^{m}, \forall \mu \in \Lambda .
\end{aligned}
$$

Individual rationality is fulfilled if also the following constraints hold:

$$
\sum_{\lambda^{-j} \in \Lambda^{m-1}}\left[v\left(\alpha\left(\lambda_{j}, \lambda^{-j}\right), \lambda_{j}\right)-p_{j}\left(\lambda_{j}, \lambda^{-j}\right)\right] P\left(\lambda^{-j}\right) \geq 0, \quad \forall j \in\{1, \ldots, m\}, \forall \lambda \in \Lambda^{m} .
$$

The objective of the auctioneer is to maximize expected revenue:

$$
\max _{\alpha, p} \sum_{\lambda \in \Lambda^{m}}\left[\sum_{j=1}^{m} p_{j}(\lambda)\right] P(\lambda) .
$$

To simplify these expressions we define the following:

$$
\bar{v}\left(\mu \mid \lambda_{\diamond}, \alpha\right)=\sum_{\lambda^{-j} \in \Lambda^{m-1}} v\left(\alpha\left(\mu, \lambda^{-j}\right), \lambda_{j}\right) P\left(\lambda^{-j}\right)
$$

where we used the bidder-neutral $\lambda_{\diamond} \in \Lambda$ as the expected value is the same for all bidders; and

$$
\bar{p}(\mu)=\sum_{\lambda^{-j} \in \Lambda^{m-1}} p_{j}\left(\mu, \lambda^{-j}\right) P\left(\lambda^{-j}\right)
$$

which is bidder-neutral for the same reason. Using these definitions the auctioneer's problem can be summarized in the following program:

$$
\max _{\alpha, \bar{p}} m \sum_{\lambda_{\diamond} \in \Lambda} \bar{p}\left(\lambda_{\diamond}\right)
$$

subject to:

(RMA)

$$
\begin{aligned}
\bar{p}\left(\lambda_{\diamond}\right)-\bar{p}(\mu) & \leq \bar{v}\left(\lambda_{\diamond} \mid \lambda_{\diamond}, \alpha\right)-\bar{v}\left(\mu \mid \lambda_{\diamond}, \alpha\right), \quad \forall \lambda_{\diamond}, \mu \in \Lambda, \\
\bar{p}\left(\lambda_{\diamond}\right) & \leq \bar{v}\left(\lambda_{\diamond} \mid \lambda_{\diamond}, \alpha\right), \quad \forall \lambda_{\diamond} \in \Lambda .
\end{aligned}
$$


Note that it is possible to integrate the second set of constraints (rationality constraints) in the incentive compatibility constraints by defining a dummy-bidder in the same way as we did for (MON). Then, (RMA) is (the dual formulation of) a parametric shortest path tree problem with (parametric) arc-length function $\bar{v}\left(\lambda_{\diamond} \mid \lambda_{\diamond}, \alpha\right)-\bar{v}\left(\mu \mid \lambda_{\diamond}, \alpha\right)$.

The example in this section is from the survey on combinatorial auctions of De Vries and Vohra [54]. 


\title{
Chapter 7
}

\section{Summary and Conclusions}

\author{
"There is something about all this that I do not understand; \\ but if ever we need to know it, you may be sure that we shall." \\ The Hermit in 'The Horse and His Boy' - C.S. Lewis
}

In this thesis we focussed on incentive alignment between decision makers in supply chains. We considered the multi-period production planning problems of these decision makers and the way how short term operational transactions between them can be chosen such that supply chain costs are minimized. The complicating factor in these situations is that different decision makers have different goals in mind and can not be forced to behave in a social way.

The VCG mechanism is a well known mechanism to align incentives between different actors, however, in Example 1.4.1 we showed that in single supplier-single buyer relations the mechanism may lead to budget deficits that are equal to the cost benefits from incentive alignment. This means that a third party has to pay an amount of money to the buyer and supplier that equals the total cost savings faced by them. However, such a benefactor may not exist and incentive alignment is only implementable if the cost reduction itself is the incentive to cooperate. As the single supplier-single buyer setting faces a budget deficit, we use auction mechanisms where multiple suppliers compete for supplying goods.

In chapter 2 we introduced a combinatorial auction for a multi-period procurement setting, where the buyer in the supply chain procures its input via an auction. We proved that the combinatorial auction is appropriate for this setting, i.e. it fits the cost structure implied by the planning problems faced by the bidders. These planning prob- 
lems are modeled as lot-sizing problems. The combinatorial auction allows bidders to fully incorporate their cost structure, in particular balancing setup and holding costs. As a consequence, a combinatorial auction using the rules of the VCG mechanism will yield the cost minimizing production plan of the lot-sizing problem. By two examples we showed that even though the combinatorial auction allows bidders to express their costs appropriately in their bids, it might be better for the auctioneer to use less sophisticated auction mechanisms. Two alternative auction mechanisms have a strong relation to the extreme solutions of the lot-sizing problem. A first alternative is to have one auction that procures all demand in the first period, leaving the auctioneer with the highest possible inventories. The second alternative is to organize an auction in each period separately and preclude the possibility of inventories. We showed the counterintuitive result that either of the alternative auction formats, that do limit production flexibility, might be cheaper for the auctioneer than the combinatorial procurement auction. However, we also show that for the two-period setting, the combinatorial auction seems to be an attractive compromise between the alternatives as it is never the most expensive of the tree.

Chapter 3 compared the procurement costs of the combinatorial auction and the alternative of organizing an auction in each period separately. The combinatorial auction will increase production efficiency as compared to the series of separate auctions, as it will allow the suppliers to incorporate more information about their costs. In other words, suppliers can produce more cheaply and therefore bid more competitively against each other. Moreover, a combinatorial auction will allow the buyer to purchase units in advance of when required. This can be advantageous if supplier production capacity is scarce in some periods, or if production costs vary from period to period. We showed that if suppliers are not limited by capacities, the combinatorial auction dominates the separate auctions as it is never more expensive for the auctioneer than the separate auctions. However, from Example 2.3.2 we know that there are capacitated cases in which the buyer will be worse off using a combinatorial auction. So, in spite of the increase in production efficiency the buyer needs in fact to pay more. The main result of this chapter is that for a two-period scenario the combinatorial auction dominates the alternative in expectation.

Chapter 4 compared the procurement costs of the combinatorial auction and the other alternative, called stock auction. The stock auction allows suppliers to achieve economies of scale, but gives no flexibility in production timing. The combinatorial auction gives this flexibility and therefore decreases production costs compared to the stock auction. Similar to the result of the previous chapter, we show that if suppliers are not limited by capacities, the combinatorial auction dominates the stock auction as 
it is never more expensive for the auctioneer than the stock auction. However, from Example 2.3.3 we know that there are capacitated cases in which the buyer will be worse off using a combinatorial auction instead of the stock auction. So, in spite of the increase in production efficiency the buyer needs in fact to pay more. The main result of this chapter is that in a two-period scenario, the possible loss from using the combinatorial auction instead of the stock auction can be limited in two ways. First, this loss is at most one setup cost. Second, we showed that the loss depends on the level of competition between suppliers. More specifically, the loss is limited by the difference in unit production costs of the two cheapest suppliers in the second period.

In chapter 5 we applied the VCG mechanism to the capacitated assignment problem, where buyers can bid for collections of items but the valuations are linear within capacity restrictions. We showed that the TU-game corresponding to the capacitated assignment problem is concave, which implies the buyers-are-substitutes property. Furthermore, we showed that the capacitated assignment problem is a special case of the models discussed by Bikhchandani and Ostroy [4] and De Vries et al. [53]. As the main results of Bikhchandani and Ostroy [4] and De Vries et al. [53] are conditional to respectively the buyers-are-substitutes property and concavity, their results can be applied to the capacitated assignment problem. Specifically, this means that the VCG mechanism corresponds to a price equilibrium, and that the VCG mechanism can equivalently be replaced by the primal-dual auction. We extended the assignment problem with capacities in two directions. First, we studied the generalized assignment problem where items have different sizes. Second, we added setup costs to the buyers' valuations. For both extensions, the buyers-are-substitutes condition and therefore concavity does not hold in general as we showed by examples. This means that even for very straightforward extensions of the assignment problem we cannot ensure that the outcome of the VCG mechanism is supported by a price equilibrium, or can be achieved by an ascending auction. Finally, we also showed that it may be necessary to have non-anonymous prices in the price equilibrium that corresponds to the VCG mechanism.

Chapter 6 discussed a parametric shortest path tree problem. The problem is a basic version of a problem class for designing incentive compatible and individual rational mechanisms, for example in bargaining in the EOQ setting or in auctions. The problem is a generalization of the shortest path problem. The parametric problem is a shortest path problem where the lengths of the arcs are defined by the products of arc dependent constants and node dependent variables. We showed that although the parametric problem is nonlinear, it can be reformulated as a linear programming problem by using duality theory, and is therefore polynomially solvable. However, we searched for an algorithm that uses the combinatorial structure of the problem allowing for faster op- 
timization. First we proved an optimality condition for the shortest path tree problem in terms of parameter values and the set of arcs that are part of a shortest path. This condition has the nice property that it is in fact a local condition. The optimality condition suggests a local improvement algorithm, but unfortunately, the local improvement algorithm may not be finite, for which we gave an example. Second, we showed an alternative formulation for the parametric shortest path tree problem where, surprisingly, the parametric variables can be eliminated. However, we could still construct an example for which local improvement is infinite. The existence of a combinatorial algorithm that is running in polynomial time remains an open question.

This thesis focussed on incentive alignment between different decision makers in supply chains. More specifically, we considered short term operational coordination of the transactions between links in a supply chain. Among the many aspects of these transactions, we considered only two of them, namely, production planning in relation to cost minimization, and the incentives that the actors have to behave strategically. As stated in many quotes from scientific literature, this field is a very difficult and mainly uncultivated field at the frontiers of knowledge. We explored some of the problems from this field and showed both possibilities and challenges. Our main suggestion for coordination is the use of procurement auctions in the multi-period production setting that is described by the lot-sizing model. This mechanism avoids the budgetary problems that exist in bilateral exchange situations and allows for optimal matching of production timing and demand. Moreover, on the average, the increased efficiency of supply chain planning appears to be profitable for both suppliers and buyer. The main challenge is to put these kind of mechanisms into practice. 


\section{Bibliography}

[1] Lawrence M. Ausubel and Paul Milgrom. The lovely but lonely Vickrey auction. In Peter Cramton, Yoav Shoham, and Richard Steinberg, editors, Combinatorial Auctions, chapter 1, pages 17-39. MIT Press, Cambridge, MA, 2006.

[2] Avijit Banerjee. A joint economic-lot-size model for purchaser and vendor. Decision Sciences, 17(3):292-311, 1986.

[3] Martin Bichler, Andrew Davenport, Gail Hohner, and Jayant Kalagnanam. Industrial procurement auctions. In Peter Cramton, Yoav Shoham, and Richard Steinberg, editors, Combinatorial Auctions, chapter 23, pages 593-611. MIT Press, Cambridge, MA, 2006.

[4] Sushil Bikhchandani and Joseph M. Ostroy. The package assignment model. Journal of Economic Theory, 107(2):377-406, December 2002.

[5] Nadjib Brahimi, Stéphane Dauzere-Peres, Najib M. Najid, and Atle Nordli. Single item lot sizing problems. European Journal of Operational Research, 168:1-16, 2006.

[6] Gérard P. Cachon and Serguei Netessine. Game theory in supply chain analysis. In David Simchi-Levi, S. David Wu, and Zuo-Jun (Max) Shen, editors, Handbook of Quantitative Supply Chain Analysis, Modeling in the E-Business Era, chapter 2, pages 13-65. Kluwer Academic Publishers, Boston, 2004.

[7] Edward H. Clarke. Multipart pricing of public goods. Public Choice, 11:17-33, 1971.

[8] Peter Cramton, Yoav Shoham, and Richard Steinberg, editors. Combinatorial Auctions. MIT Press, Cambridge, MA, 2006.

[9] Gregor Dudek and Hartmut Stadtler. Negotiation-based collaborative planning between supply chains partners. European Journal of Operational Research, 163(3):668-687, 2005. 
[10] Wedad Elmaghraby. Auctions and pricing in e-marketplaces. In David Simchi-Levi, S. David Wu, and Zuo-Jun (Max) Shen, editors, Handbook of Quantitative Supply Chain Analysis, Modeling in the E-Business Era, chapter 6, pages 213-246. Kluwer Academic Publishers, Boston, 2004.

[11] Wedad Elmaghraby. The effect of asymmetric bidder size on an auction's performance: Are more bidders always better? Management Science, 51(12):17631776, December 2005.

[12] Donald Erlenkotter. Ford Whitman Harris and the economic order quantity model. Operations Research, 38(6):937-946, November-December 1990.

[13] EURO. The Association of European Operational Research Societies, website. http://www.euro-online.org, 2007.

[14] Michael Florian and Morton Klein. Determining production planning with concave costs and capacity constraints. Management Science, 18(1):12-20, September 1971.

[15] Drew Fudenberg and Jean Tirole. Game theory. MIT Press, Cambridge, MA, 1991.

[16] Game Theory Society. Website. http://www.gametheorysociety.org, 2007.

[17] Paul M. Griffin and Christina R. Scherrer. Next generation ERP systems: Scalable and decentralized paradigms. In David Simchi-Levi, S. David Wu, and ZuoJun (Max) Shen, editors, Handbook of Quantitative Supply Chain Analysis, Modeling in the E-Business Era, chapter 18, pages 747-782. Kluwer Academic Publishers, Boston, 2004.

[18] Theodore Groves. Incentives in teams. Econometrica, 41(4):617-631, July 1973.

[19] Faruk Gul and Ennio Stacchetti. Walrasian equilibrium with gross substitutes. Journal of Economic Theory, 87(1):95-124, July 1999.

[20] Ford W. Harris. How many parts to make at once. Factory, The Magazine of Management, 10(2):135-136, 152, February 1913. Reprinted in 1990 [21].

[21] Ford W. Harris. How many parts to make at once. Operations Research, 38(6):947950, November-December 1990. Reprint of Harris [20].

[22] Oliver D. Hart. On the optimality of equilibrium when the market structure is incomplete. Journal of Economic Theory, 11(3):418-443, December 1975. 
[23] Stan van Hoesel, H. Edwin Romeijn, Dolores Romero Morales, and Albert P. M. Wagelmans. Integrated lot sizing in serial supply chains with production capacities. Management Science, 51(11):1706-1719, November 2005.

[24] Gail Hohner, John Rich, Ed Ng, Grant Reid, Andrew J. Davenport, Jayant R. Kalagnanam, Ho Soo Lee, and Chae An. Combinatorial and quantity-discount procurement auctions benefit Mars, Incorporated and its suppliers. Interfaces, 33(1):23-35, January-February 2003.

[25] John H. Kagel and Dan Levin. Behavior in multi-unit demand auctions: experiments with uniform price and dynamic Vickrey auctions. Econometrica, 69(2):413-454, March 2001.

[26] S. Kameshwaran, L. Benyoucef, and X. Xie. Discount auctions for procuring heterogeneous items. In Proceedings of the 7th international conference on Electronic commerce, pages 244-249, New York, NY, 2005. ACM Press.

[27] B. Karimi, S. M. T. Fatemi Ghomi, and J. M. Wilson. The capacitated lot sizing problem: a review of models and algorithms. Omega, 31(5):365-378, October 2003.

[28] Benny Lehmann, Daniel Lehmann, and Noam Nisan. Combinatorial auctions with decreasing marginal utilities. Games and Economic Behavior, 55(2):270-296, May 2006.

[29] Herman B. Leonard. Elicitation of honest preferences for the assignment of individuals to positions. Journal of Political Economy, 91(3):461-479, 1983.

[30] Reinder B. Lok, Dolores Romero Morales, and Dries Vermeulen. Note on the applicability of the VCG mechanism to capacitated assignment problems and extensions. Statistica Neerlandica, 61(1):156-171, February 2007.

[31] Andreu Mas-Colell, Michael D. Whinston, and Jerry R. Green. Microeconomic Theory. Oxford University Press, New York, 1995.

[32] Christian Montet and Daniel Serra. Game Theory and Economics. Palgrave Macmillan, New York, 2003.

[33] Roger B. Myerson and Mark A. Satterthwaite. Efficient mechanisms for bilateral trading. Journal of Economic Theory, 29(2):265-281, April 1983.

[34] V. G. Narayanan and Ananth Raman. Aligning incentives in supply chains. Harvard Business Review, November 2004. DOI: 10.1225/R0411F. 
[35] George L. Nemhauser and Laurence A. Wolsey. Integer and Combinatorial Optimization. John Wiley \& Sons, New York, 1988.

[36] NGB. Nederlands Genootschap voor Besliskunde, website. http://www.ngbonline.nl/besliskunde.php, 2007.

[37] Stefano Pallottino and Maria Grazia Scutellà. Dual algorithms for the shortest path tree problem. Networks, 29(2):125-133, March 1997.

[38] Christos H. Papadimitriou. Computational complexity. Addison-Wesley, Reading, MA, 1994.

[39] Christos H. Papadimitriou and Kenneth Steiglitz. Combinatorial Optimization: Algorithms and Complexity. Dover Publications, Mineola, New York, second edition, 1998.

[40] Aleksandar Pekeč and Michael H. Rothkopf. Combinatorial auction design. Management Science, 49(11):1485-1503, November 2003.

[41] Edieal J. Pinker, Abraham Seidmann, and Yaniv Vakrat. Managing online auctions: Current business and research issues. Management Science, 49(11):1457-1484, November 2003.

[42] Jean-Charles Rochet and Lars A. Stole. The economics of multidimensional screening. In Mathias Dewatripont, Lars Peter Hansen, and Stephen J. Turnovsky, editors, Advances in Economics and Econometrics, chapter 5, pages 150-197. Cambridge University Press, 2003.

[43] Vijay K. Rohatgi and A.K.Md. Ehsanes Saleh. An Introduction to Probability and Statistics. Wiley series in probability and statistics. John Wiley \& Sons, New York, 2nd edition, 2001.

[44] Dolores Romero Morales, Reinder B. Lok, and Richard Steinberg. Combinatorial auctions for the procurement of a product over a planning horizon. In: Fifth ALIO/EURO Conference on Combinatorial Optimization, 26-28 October 2005, Paris, France.

[45] Dolores Romero Morales, Reinder B. Lok, and Richard Steinberg. Joint versus serial auctions in procurement. In: EURO XXI, European Conference on Operational Research, 2-5 July 2006, Reykjavik, Iceland.

[46] Lloyd S. Shapley. Complements and substitutes in the optimal assignment problem. Naval Research Logistics Quarterly, 9:45-48, 1962. 
[47] Régine Slagmulder, Daniel Corsten, and Dan Grottoli. Sainsbury's (A): Transforming the supply chain. Case 603-020-1, INSEAD/University of St Gallen, France/Zwitserland, 2003.

[48] Régine Slagmulder, Daniel Corsten, and Dan Grottoli. Sainsbury's (B): Supply chain performance measurement. Case 103-057-1, INSEAD/University of St Gallen, France/Zwitserland, 2003.

[49] Eric Sucky. A bargaining model with asymmetric information for a single suppliersingle buyer problem. European Journal of Operational Research, 171(2):516-535, 2006.

[50] Donald M. Topkis. Supermodularity and Complementarity. Frontiers of Economic Research. Princeton University Press, Princeton, New Jersey, 1998.

[51] Tunay I. Tunca and Qiong Wu. Multiple sourcing and procurement sourcing selection with bidding events. Working paper, Stanford University, Graduate School of Business, September 2006.

[52] William Vickrey. Counterspeculation, auctions, and competitive sealed tenders. The Journal of Finance, 16(1):8-37, March 1961.

[53] Sven de Vries, James Schummer, and Rakesh V. Vohra. On ascending Vickrey auctions for heterogeneous objects. Journal of Economic Theory, 132(1):95-118, January 2007.

[54] Sven de Vries and Rakesh V. Vohra. Combinatorial auctions: A survey. INFORMS Journal on Computing, 15(3):284-309, 2003.

[55] Harvey M. Wagner and Thomson M. Whitin. Dynamic version of the economic lot size model. Management Science, 5(1):89-96, October 1958. Reprinted in 2004 [56].

[56] Harvey M. Wagner and Thomson M. Whitin. Dynamic version of the economic lot size model. Management Science, 50(12S):1770-1774, December 2004. Reprint of Wagner and Whitin [55].

[57] Candace A. Yano and Stephen M. Gilbert. Coordinated pricing and production / procurement decisions: A review. In Amiya K. Chakravarty and Jehoshua Eliashberg, editors, Managing Business Interfaces: Marketing, Engineering and Manufacturing Perspectives, chapter 3, pages 65-103. Kluwer Academic Publishers, Dordrecht, 2004. 


\section{Index}

$\mathrm{AF}, 114$

APC, 87

APCS, 97

assignment problem, 84, 86, 92, 94

generalized -, 95

auction

combinatorial -, 27, 35, 36, 39, 53, 71, 83

definition, 22

definition, 20

Dutch -, 21

English -, 20, 22, 40, 84

procurement -, 27, 39, 57

series of single-period $-\mathrm{s}, 40,41,45$, 46, 53

stock -, 35, 41, 71

definition, 45

Vickrey -, 21, 25

Ausubel, L.M., 83

Banerjee, A., 32

bargaining model, 32, 101

Bichler, M., 39, 40

Bikhchandani, S., 36, 84, 85, 90-92, 123, 139

Brahimi, N., 16

buyers-are-substitutes property, 84, 85, 89 definition, 85

Cachon, G.P., 20, 23

Clarke mechanism, 26

Clarke, E.H., 25, 26 combinatorial auction, see auction, combinatorial

concave, 85

definition, 84

concavity, see concave

coordination, $11,17,28$

central -, 31

core, 85, 91

Cramton, P., 22

De Vries, S., see Vries, S. de

descending clock auction, see auction, Dutch dominant strategy, 20

weakly -, 20

Dual-SPT, 103

Dudek, G., 13, 17, 28-31

Dutch auction, see auction, Dutch

economic lot-size model, see lot-sizing problem

economic ordering quantity, see EOQ model

efficiency, 12, 18, 24, 40, 53

$$
\text { Pareto -, } 18
$$

Ehsanes Saleh, A.K.Md., 65

Elmaghraby, W., 40, 57

English auction, see auction, English

EOQ model, 13, 14, 32

equilibrium

Bayes-Nash -, 23, 119

definition, 20

minimal price -

definition, 90 


$$
\begin{aligned}
& \text { Nash -, } 93 \\
& \text { price -, } 84 \\
& \text { definition, } 90
\end{aligned}
$$

Erlenkotter, D., 15

EURO, 13

exchange economy, 83, 90

Florian, M., 15

Fudenberg, D., 20

game, 20

definition, 19

screening -, 34, 101, 118

definition, 23

TU-game

definition, 85

game theory, 11,13

applications, 20

cooperative,- 85

definition, 17

GAP, 95

Gilbert, S.M., 39

Griffin, P.M., 29

gross substitutes property, see substitutes, gross

Groves mechanism, 26

Groves, T., 25, 26

Gul, F., 89

Harris, F.W., 15

Hart, O.D., 53, 71, 72

Hoesel, C.P.M. van, 13

Hohner, G., 22, 39, 40

i.i.d., 59

incentive, 11, 12

incentive compatible, 22, 24, 25, 31, 34

joint capacity effect, 54-56

Kagel, J.H., 84
Kameshwaran, S., 41

Karimi, B., 16

Klein, M., 15

Lehman, B., 89

Leonard, H.B., 84, 98

Levin, D., 84

Lewis, C.S., 11, 121

local improvement algorithm, 107 definition, 109

Lok, R.B., 41, 84

lot-sizing problem, 13, 15, 29, 39, 42

extreme solutions, 45

multi-level -, 29

single-item -, 15, 16

LP, 104

LSP, 16

marginal contribution, 26, 44

marginal product, 26, 44

Mas-Colell, A., 19, 20, 23, 25-27

mechanism, 24

definition, 17, 20, 22

desirable characteristics, 24

VCG -, 25, 44

definition, 26

properties, 27

mechanism design, 13, 17, 20

definition, 22

Milgrom, P., 83

MLSP, 29

MON, 118

Montet, C., 20, 22, 25

Myerson, R.B., 25

Narayanan, V.G., 12

Nemhauser, G.L., 86, 102

Netessine, S., 20, 23

NGB, 136 
Operational Research, 12

optimization, 12, 16, 34, 101

efficiency, 18

Ostroy, J.M., 36, 84, 85, 90-92, 123, 139

overdemand, 93

Pallottino, S., 102

Papadimitriou, C.H., 101, 104

parametric shortest path tree problem, 101, 102

definition, 103

linear program, 104

optimality condition, 104, 105

payoff, 19, 24, 26

PD auction, 92

definition, 93

Pekeč, A., 22

Pinker, E.J., 40

pivotal mechanism, 26

players-are-substitutes property, see buyers-

price are-substitutes property

$$
\begin{array}{r}
\text { linear -, 84, } 98 \\
\text { definition, } 99
\end{array}
$$

price equilibrium, see equilibrium, price prices

$$
\begin{gathered}
\text { anonymous -, 95, } 99 \\
\text { definition, } 99
\end{gathered}
$$

primal dual auction, see PD auction

prisoner's dilemma, 19

PSPT, 104

Raman, A., 12

RMA, 119

Rochet, J.-C., 118

Rohatgi, V.K., 65

Romero Morales, D., 13, 41, 84

Rothkopf, M.H., 22
Satterthwaite, M.A., 25

Scherrer, C.R., 29

Schummer, J., 36, 84, 85, 90, 92-94, 123, 139

screening game, see game, screening screening problem, see game, screening Scutellà, M.G., 102 second-price sealed-bid auction, see auction, Vickrey

Serra, D., 20, 22, 25

SG, 34

Shapley, L.S., 85, 88

shortest path problem

definition, 102

Slagmulder, R., 57

Smith, A., 12

social welfare

definition, 18

function, 18

utilitarian - function, 18

SPT, 103

Stacchetti, E., 89

Stadtler, H., 13, 17, 28-31

Steiglitz, K., 101

Steinberg, R., 22, 41

stock auction, see auction, stock

Stole, L.A., 118

submodular, 84

substitutes, 84-86

gross - property, 89

definition, 89

Sucky, E., 14, 16, 28, 29, 32-34, 101

supply chain, 11

assumption on inventory costs, 44

cooperation, 12

definition, 11

management, 12, 40 
performance, 12

production planning in $-\mathrm{s}, 14,28$

TBO, 14

Tirole, J., 20

Topkis, D.M., 85, 89

transformation effect, 54-56

truthful report, 23

truthtelling, 21

TU-game, see game, TU-game

Tunca, T.I., 40

undersupplied, 93

minimally -, 93

upstream planning, 30, 31

utility

definition, 17

function, 17, 19, 117

quasi-linear,- 24

quasi-linear - function, 117, 118

valuation

linear -, 84, 97

monetary -, 17, 117

net -, 24, 26

notation, 24

total,- 24

Van Hoesel, C.P.M., see Hoesel, C.P.M. van

VCG mechanism, see mechanism, VCG

Vermeulen, D, 84

Vickrey auction, see auction, Vickrey

Vickrey payment, 26

Vickrey, W., 21, 25, 28

Vohra, R.V., 36, 84, 85, 90, 92-94, 123, 139

Vries, S. de, 36, 84, 85, 90, 92-94, 123, 139

Wagner, H.M., 15, 34
Whitin, T.M., 15, 34

Wolsey, L.A., 86, 102

Wu, Q., 40

Yano, C.A., 39 



\section{Samenvatting}

\section{Veilingmechanismen voor het optimaliseren van de pro- ductieketen}

\section{Achtergrond}

Dit proefschrift gaat over het plannen van productie en voorraad als de vraag naar de producten bekend is en vast ligt. Uitgangspunt is dat de productie plaatsvindt op verschillende momenten en dat de kosten bestaan uit vaste kosten voor het opstarten van de productie, variabele kosten voor iedere geproduceerde eenheid, en voorraadkosten voor producten die eerder geproduceerd worden dan ze nodig zijn. Stel dat een producent weet hoeveel eenheden van het product hij in iedere periode kan afzetten. In dat geval wil hij weten hoe hij dat op de goedkoopste manier kan produceren. Aan de ene kant moet de producent zo veel mogelijk in een keer produceren, om de vaste kosten zo laag mogelijk te houden. Immers, bij verschillende productieseries worden voor iedere serie opnieuw de vaste kosten gemaakt. Helaas heeft deze strategie het nadeel dat de producten die pas over enige tijd gebruikt worden, in voorraad moeten worden gehouden. Aan de andere kant zou de producent daarom zoveel mogelijk precies op tijd moeten produceren. In veel gevallen zal de goedkoopste optie tussen de twee uitersten van 'alles in een keer produceren' en 'alles precies op tijd produceren' in liggen. In essentie is het probleem dus om te bepalen hoe groot de productieseries op ieder moment moeten zijn om de totale kosten zo laag mogelijk te krijgen. We zullen dit probleem daarom aanduiden als het seriegrootteprobleem.

Het seriegrootteprobleem kent twee basisvarianten, een voor goederen die (min of meer) oneindig deelbaar zijn, zoals olie, en een voor productie die noodzakelijk per stuk worden gemaakt, zoals bijvoorbeeld mobiele telefoons. De eerste variant werd in 1913 voor het eerst in een wiskundig model geformuleerd door Harris [20]. Bijna een halve eeuw later presenteerden Wagner en Whitin [55] een model voor de tweede variant. Beide modellen zijn voorbeelden van problemen uit het vakgebied van de 
(mathematische) besliskunde ${ }^{1}$, en zijn daarin uitgebreid bestudeerd. Zie bijvoorbeeld de overzichten van Erlenkotter [12], Brahimi et al. [5] en Karimi et al. [27].

In dit proefschrift kijken we naar het seriegrootteprobleem vanuit het perspectief van de productieketen (supply chain) waarin de ene producent een halffabrikaat levert aan een volgende producent die het halffabrikaat verwerkt tot eindproduct. In deze situatie hebben zowel de leverancier als de afnemer hun eigen seriegrootteprobleem. In beginsel zullen beiden hun productie zo willen plannen dat hun eigen totaal van vaste, variabele, en voorraadkosten minimaal is. Maar, producenten kunnen het zich niet veroorloven om alleen naar hun eigen kosten te kijken. Voor de concurrentiepositie van het eindproduct zijn de totale kosten van de hele productieketen van belang. Narayanan en Raman [34] maken duidelijk dat er alleen verliezers zullen zijn als de partners in een productieketen niet samenwerken. De vraag is dan hoe de verschillende schakels van een productieketen kunnen samenwerken. Eén oplossing is de fusie van beide producenten zodat we niet meer te maken hebben met twee op elkaar af te stemmen planningsproblemen, maar met één geïntegreerd planningsprobleem. Het bijbehorende tweelaagse seriegrootteprobleem is eveneens een veel bestudeerd probleem in de besliskunde, zie bijvoorbeeld het werk van Van Hoesel et al. [23]. Een andere nog nauwelijks bestudeerde oplossingsrichting is die van het formuleren van (afdwingbare) spelregels waarbinnen de producenten tot een gezamenlijke planning komen maar toch zelfstandig blijven beslissen. In deze richting hebben we niet meer alleen te maken met een optimaliseringsprobleem, maar ook met de strategische interactie tussen de producenten. Deze strategische interactie wordt uitgebreid bestudeerd in het vakgebied van de speltheorie, en wordt een spel genoemd. Een praktisch voorbeeld van een spel is een veiling zoals de bloemenveiling in Eelde, waar het mechanisme van de veiling wordt gebruikt om bloemen te verkopen. Andersom gebruiken steeds meer bedrijven (omgekeerde) veilingen om goederen in te kopen, bijvoorbeeld Mars, Inc. (Masterfoods), zie de studie van Hohner et al. [24]. Volgens Elmaghraby [10] is de interactie van kostenminimalisatie van de leveranciers en het ontwerp van de inkoopveiling een nog onontgonnen gebied in de wetenschap. Dit is het gebied waar we in dit proefschrift enkele voorzichtige stappen zetten: Veilingmechanismen voor het optimaliseren van de productieketen.

\footnotetext{
${ }^{1}$ Het Nederlands Genootschap voor Besliskunde [36] beschrijft het vakgebied als volgt: "Besliskunde omvat de theorie en toepassing van wiskundige technieken voor het modelleren en verbeteren van processen in bedrijven en andere organisaties. (..) Besliskunde bevindt zich (..) op het snijvlak van Wiskunde, Bedrijfskunde en Informatica. Besliskunde wordt ook wel Operations Research, of Management Science genoemd."
} 


\section{Het proefschrift}

Samenvattend kunnen we zeggen dat in dit geschrift het volgende probleemgebied centraal staat.

- Planning van productie en voorraad, het zogenaamde seriegrootteprobleem,

- waarin we kijken naar de afstemming van de individuele beslissingsproblemen van opeenvolgende schakels in een productieketen, en

- waarvoor de spelregels moeten worden ontworpen zodat de individuele belangen van de opeenvolgende schakels samenvallen met het belang van de hele keten.

Een bekend mechanisme om de belangen van verschillende spelers met elkaar te verzoenen is het VCG-mechanisme. Onderdeel van dit mechanisme zijn betalingen aan of van de spelers. Deze betalingen zijn zodanig dat alle spelers er belang bij hebben om open kaart te spelen en daarmee gezamenlijk de ketenbrede goedkoopste productieplanning te kiezen. Om in de setting van de productieketen te kunnen worden gebruikt, moeten de betalingen wel zo zijn dat er een overschot is op de begroting. Dat wil zeggen dat de betalingen aan de ene groep spelers betaald moet kunnen worden met de betalingen van de andere spelers. We tonen met een voorbeeld aan dat de toepassing van dit mechanisme op de setting met één leverancier en één afnemer, helaas, een fors begrotingstekort heeft. Om dit probleem te omzeilen, breiden we het aantal leveranciers uit zodat we met een veilingversie van het VCG-mechanisme kunnen werken.

In hoofdstuk 2 introduceren we een combinatorische veiling voor de setting waarin één afnemer via een veiling een of meer leveranciers kiest uit een groep bieders. De veiling heet combinatorisch omdat de potentiële leveranciers biedingen mogen uitbrengen op een bepaalde combinaties van de gevraagde hoeveelheden. We tonen aan dat de leveranciers op deze manier de kostenstructuur uit het seriegrootteprobleem exact kunnen vertalen in passende biedingen. Bovendien, door gebruik te maken van de betalingsregels van het VCG-mechanisme, is het voor alle leveranciers het beste om hun werkelijke kosten te bieden. Op die manier kan de veilingmeester (in dit geval de afnemer) de combinatie van biedingen kiezen die de laagste totale kosten geeft. Hoewel de combinatorische veiling dus precies doet wat we willen, namelijk het minimaliseren van de kosten van de hele keten, is er toch een probleem. Met twee voorbeelden tonen we aan dat in bepaalde gevallen de afnemer beter af kan zijn met minder geavanceerde veilingen. We hebben dus een mooi mechanisme wat precies doet wat we willen, maar de vraag is of de afnemer daadwerkelijk zal kiezen voor dit mechanisme. Het eerste voorbeeld gebruikt de aanname dat de afnemer kiest om alle benodigde halffabrikaten al in de eerste periode in te kopen. In dat geval heeft de afnemer dus een maximale 
voorraad. Het tweede voorbeeld gebruikt dat de afnemer voor iedere periode apart een veiling organiseert en geen voorraden aanhoudt. Een bemoedigend resultaat is dat hoewel deze alternatieve veilingen in sommige omstandigheden het beste alternatief zijn voor de afnemer, ze ook heel slecht kunnen uitpakken. Daartegenover staat dat voor alle gevallen met maar twee perioden, de combinatorische veiling óf de goedkoopste optie is, óf de op een na goedkoopste, maar nooit de duurste van de drie.

In hoofdstuk 3 maken we een nadere vergelijking tussen de combinatorische veiling en het alternatief om in iedere periode apart een veiling te organiseren. Zoals gezegd doet de combinatorische veiling precies wat we willen, namelijk het minimaliseren van de totale kosten van de productieketen. Dit is het geval omdat de leveranciers hun echte kosten volledig kunnen uitdrukken in de soort biedingen die de afnemer accepteert, en dat zo ook daadwerkelijk hun echte kosten willen bieden omdat dat het beste voor hen is vanwege de eigenschappen van het VCG-mechanisme. Wanneer in iedere periode een aparte veiling wordt georganiseerd, dan zullen de leveranciers niet meer in staat zijn om in een keer de goedkoopste oplossing van hun seriegrootteprobleem te bepalen, simpelweg omdat ze niet van tevoren weten welke combinaties ze uiteindelijk mogen leveren. De afnemer is daardoor ook niet meer in staat om de oplossing te kiezen die de kosten van de keten minimaliseert. We laten zien dat wanneer de leveranciers niet gehinderd worden door capaciteitsbeperkingen, de afnemer altijd voor de combinatorische veiling moet kiezen. Helaas is dat niet meer waar als er wel capaciteitsbeperkingen zijn. Toch kunnen we als belangrijkste resultaat van dit hoofdstuk aantonen dat wanneer de productiekosten onafhankelijke en identiek (uniform) verdeelde stochasten zijn, en we ons beperken tot problemen met twee perioden, de combinatorische veiling voor de afnemer in verwachting beter is dan het alternatief van aparte veilingen.

In hoofdstuk 4 maken we een nadere vergelijking tussen de combinatorische veiling en het alternatief om alle halffabrikaten al in de eerste periode in te kopen. Uiteraard gelden hier dezelfde mooie eigenschappen voor de combinatorische veiling als hiervoor genoemd. In het alternatief van één veiling in de eerste periode kunnen de leveranciers wel schaalvoordelen halen, maar hebben ze niet de flexibiliteit om productiecapaciteit in latere perioden in te zetten. Ook hier geldt dus dat het alternatief vaak geen ruimte laat voor de goedkoopste oplossing voor de hele keten. We laten zien dat wanneer de leveranciers niet gehinderd worden door capaciteitsbeperkingen, de afnemer altijd voor de combinatorische veiling moet kiezen. Helaas is dat niet meer waar als er wel capaciteitsbeperkingen zijn. Toch is het mogelijke voordeel dat de afnemer kan krijgen door een inefficiënte veiling te gebruiken wel beperkt. Voor het geval met twee perioden kunnen we twee grenzen afleiden. Eerst laten we zien dat het voordeel nooit groter is dan de waarde van de vaste kosten. Daarna bewijzen we dat het voordeel afhangt 
van het niveau van de concurrentie tussen de leveranciers. Er geldt namelijk dat het voordeel ook nooit groter is dan het verschil in de per-eenheid variabele kosten van de twee goedkoopste leveranciers in de laatste periode.

In hoofdstuk 5 gaan we dieper in op de speltheoretische aspecten van het VCGmechanisme. Het VCG-mechanisme heeft een paar hele mooie theoretische eigenschappen, zoals bijvoorbeeld het hebben van dominante strategieën voor de deelnemers, en een uitkomst die gelijk is aan de optimale keuze van hetzelfde probleem met maar één beslisser. Helaas is er wel een probleem wanneer we het mechanisme in praktische situaties willen toepassen (zie ook het overzicht van Ausubel en Milgrom [1]). Het mechanisme is namelijk nogal complex in verschillende opzichten. Bijvoorbeeld, in de combinatorische veiling van de voorgaande hoofdstukken, moeten de deelnemers voor iedere mogelijke combinatie hun kosten berekenen en doorgeven aan de veiling. Het aantal mogelijke combinaties neemt echter exponentieel toe als het aantal producten toeneemt, en dit heeft als gevolg dat al voor relatief kleine problemen een veel te groot aantal biedingen moet worden gedaan en verwerkt. Deze complexiteit kan in sommige gevallen worden omzeild door een iteratieve veiling te houden die equivalent is aan het VCG-mechanisme. Een bekend voorbeeld hiervan is de Engelse veiling waarin de deelnemers elkaar om de beurt mogen overbieden. Soortgelijke alternatieven voor complexere situaties zoals de combinatorische veiling, zijn echter niet altijd equivalent aan het VCG-mechanisme. Er moet namelijk worden voldaan aan een stabiliteitsvoorwaarde gerelateerd aan het economische begrip prijsevenwicht en het wiskundige begrip submodulariteit.

We laten zien dat het VCG-mechanisme in bovenstaande zin toepasbaar is op het zogenaamde toewijzingsprobleem met capaciteiten. Dit is een eenvoudig geval van een combinatorische veiling. In deze toepassing mogen de deelnemers aan de veiling bieden op pakketten goederen. Iedere deelnemer hecht bepaalde waardes aan de individuele goederen, onafhankelijk van de pakketsamenstelling, mits het pakket zijn capaciteit niet overschrijdt. We tonen aan dat het zogenaamde spel met overdraagbaar nut (TU-game) dat hoort bij deze setting, voldoet aan de benodigde stabiliteitsvoorwaarde. Bovendien laten we zien dat dit probleem een speciaal geval is van de modellen van Bikhchandani en Ostroy [4] en De Vries et al. [53]. Helaas geldt voor twee relatief eenvoudige generalisaties van het toewijzingsprobleem dat de stabiliteitsvoorwaarde komt te vervallen. Ten eerste tonen we dit aan voor het gegeneraliseerde toewijzingsprobleem waarin de verschillende goederen van formaat kunnen verschillen (dat wil zeggen, een verschillend beslag leggen op de capaciteit van de bieders). Ten tweede wordt het ook aangetoond voor het geval waarin de bieders worden geconfronteerd met vaste kosten voor het hebben van goederen. 
In hoofdstuk 6 bespreken we een parametrische versie van het probleem om een kortste-pad-boom te vinden in een netwerk. In plaats van gegeven afstanden tussen de punten, zijn de afstanden afhankelijk van de te kiezen parameters. Dit probleem is een basale versie van de klasse van problemen waarin mechanismen worden ontworpen waarin de deelnemers vrijwillig meedoen aan het mechanisme (bijvoorbeeld een veiling) en waarin het voor hen optimaal is om te handelen in het algemeen belang. Een zo'n probleem komt voort uit het onderhandelingsmodel dat Sucky [49] heeft ontworpen voor de coördinatie in een productieketen met oneindig deelbare goederen. Als eerste laten we zien dat hoewel het probleem niet lineair is, het toch kan worden geherformuleerd als een lineair optimaliseringsprobleem. Dit impliceert dat het probleem polynomiaal oplosbaar is, wat betekent dat de maximale oplossingstijd in tegenstelling tot bijvoorbeeld de combinatorische veiling niet exponentieel toeneemt met de grootte van het probleem. Desondanks onderzoeken we in dit hoofdstuk of er een oplossingsalgoritme bestaat dat nog sneller is dan die voor gewone lineaire optimaliseringsproblemen door gebruik te maken van de specifieke structuur van het probleem. Als eerste presenteren we een optimaliteitsvoorwaarde, en laten zien dat die noodzakelijk en voldoende is voor optimaliteit. De voorwaarde heeft de mooie eigenschap dat het een lokale voorwaarde is, wat wil zeggen dat ieder punt in het netwerk aan die voorwaarde moet voldoen. Van de optimaliteitsvoorwaarde naar een eenvoudige lokale-ophogingsalgoritme is een kleine stap. We tonen aan dat dit algoritme in speciale gevallen, helaas, oneindig veel stappen vraagt. Opvallend genoeg kunnen we het parametrische probleem herformuleren door de parametrische variabelen te elimineren. Maar ook hier geldt dat een lokaal algoritme oneindig veel stappen nodig kan hebben. 


\section{About the author}

Reinder Lok was born in Groningen, The Netherlands, on the 19th of March, 1976. From 1988 to 1994 he was going to school at the Gomarus College in Groningen for his secondary education (VWO). After that, he started studying econometrics at the Rijksuniversiteit Groningen. The main subject of this study was operational research. He graduated in 1999 with a master's thesis on assigning cargo to vessels. This thesis was written during an internship at Hydrographic and Marine Consultants B.V. in Almere, The Netherlands, and was supervised by Dr. G. Sierksma. After graduation, Reinder started as a researcher in statistics at Statistics Netherlands (CBS), where he was employed until January 2003. In that year he got the opportunity to start as a $\mathrm{PhD}$ candidate at the Universiteit Maastricht. The $\mathrm{PhD}$ project was supervised by Prof. dr. ir. C.P.M. van Hoesel and Dr. M.D. Romero Morales and yielded the thesis Auction Mechanisms in Supply Chain Optimization. After finishing the manuscript in January 2007 he returned to Statistics Netherlands as researcher in statistics concerning distribution of incomes and capital. 